\title{
The Conway-Miyamoto correspondences for the Fischer 3-transposition groups
}

\author{
Ching Hung Lam* \\ Institute of Mathematics, Academia Sinica, Taipei, Taiwan 10617 \\ and National Center for Theoretical Sciences, Taipei, Taiwan \\ e-mail: chlam@math.sinica.edu.tw \\ Hiroshi Yamauchi ${ }^{\dagger}$ \\ Department of Mathematics, Tokyo Woman's Christian University \\ 2-6-1 Zempukuji, Suginami-ku, Tokyo 167-8585, Japan \\ e-mail: yamauchi@lab.twcu.ac.jp
}

2010 Mathematics Subject Classification: Primary 17B69; Secondary 20B25, 20 D08.

\begin{abstract}
In this paper, we present a general construction of 3-transposition groups as automorphism groups of vertex operator algebras. Applying to the moonshine vertex operator algebra, we establish the Conway-Miyamoto correspondences between Fischer 3-transposition groups $\mathrm{Fi}_{23}$ and $\mathrm{Fi}_{22}$ and $c=25 / 28$ and $c=11 / 12$ Virasoro vectors of subalgebras of the moonshine vertex operator algebra.
\end{abstract}

\section{Contents}

1 Introduction $\quad 2$

2 The dihedral subalgebras $r$

2.1 Virasoro vertex operator algebras . . . . . . . . . . . . . 8

2.2 2A-algebra . . . . . . . . . . . . . . . . . . 10

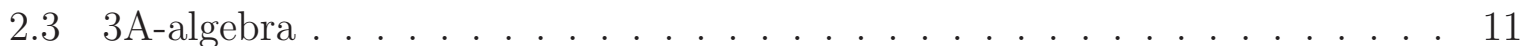

2.46 A-algebra . . . . . . . . . . . . . . . . . . 12

*Partially supported by MoST grant 104-2115-M-001-004-MY3 of Taiwan.

†Partially supported by JSPS Grant-in-Aid for Young Scientists (B) No 24740027. 
4 Inductive structures $\quad 16$

4.1 The case $\langle x, y\rangle \cong U_{2 \mathrm{~A}} \ldots \ldots \ldots \ldots \ldots$

4.2 The case $\langle x, y\rangle \cong U_{3 \mathrm{~A}} \ldots \ldots \ldots \ldots \ldots \ldots$

4.3 Inductive subalgebras . . . . . . . . . . . . . . . . . . . 26

5 Conway-Miyamoto correspondence $\quad 31$

5.1 Transpositions of $\mathrm{Fi}_{23}$ and $c=25 / 28$ Virasoro vectors . . . . . . . . . . . . 34

5.2 Transpositions of $\mathrm{Fi}_{22}$ and $c=11 / 12$ Virasoro vectors . . . . . . . . . . . 38

$\begin{array}{ll}\text { Appendix A Explicit constructions } & 39\end{array}$

A.1 Compact real form of a lattice VOA . . . . . . . . . . . . . . . . 39

A.2 Virasoro vectors associated to root systems . . . . . . . . . . . . 40

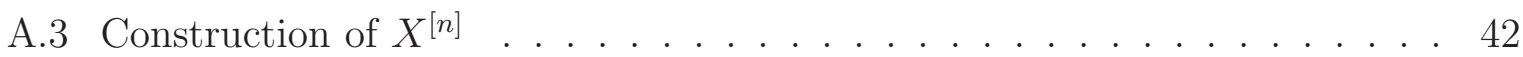

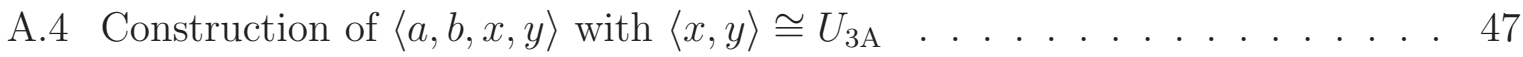

\section{Introduction}

A 3-transposition group is a pair $(G, D)$ of a group $G$ and a set of involutions $D$ of $G$, called 3-transpositions, such that $G=\langle D\rangle, D=D^{G}$ and $|a b| \leq 3$ for any $a, b \in D$. A subgroup $H$ of $G$ is called a $D$-subgroup if $H=\langle H \cap D\rangle$ which is again a 3-transposition group. Let $a \in D$ and set $D_{a}=\{x \in D \mid a x=x a\}$. The $D$-subgroup $G_{a}=\left\langle D_{a}\right\rangle$ is uniquely determined up to conjugation if $D$ is a conjugacy class in $G$. We call the 3-transposition group $G_{a} /\langle a\rangle$ an inductive structure of $G$. Given a 3-transposition group $(G, D)$, the intersection $D \cap P$ of $D$ with a Sylow 2-subgroup $P$ of $G$ is called a basic set which is a maximal subset of $D$ consisting of mutually commutative elements. The size of a basic set is called the width of $G$. Since $D^{G}=D$, the width is an invariant of a 3-transposition group. 3-transposition groups were first studied by Fischer [Fi71] (see also [As97, CH95]). Fischer also discovered the Fischer groups $\mathrm{Fi}_{22}, \mathrm{Fi}_{23}$ and $\mathrm{Fi}_{24}$ in the classification of almost simple 3-transposition groups. It turns out that Fischer groups $\mathrm{Fi}_{22}$ and $\mathrm{Fi}_{23}$ are obtained by taking inductive structures of $\mathrm{Fi}_{23}$ and $\mathrm{Fi}_{24}$, respectively, whereas the largest Fischer group $\mathrm{Fi}_{24}$ is best understood via the inclusion of its cover 3.Fi $i_{24}$ into the Monster (cf. Section 11.34 of [As97]). The subscripts of $\mathrm{Fi}_{22}, \mathrm{Fi}_{23}$ and $\mathrm{Fi}_{24}$ indicate their widths, and the normalizers of the elementary abelian subgroups generated by basic sets of $\mathrm{Fi}_{22}, \mathrm{Fi}_{23}$ and $\mathrm{Fi}_{24}$ are $2^{10}: M_{22}, 2^{11} \cdot M_{23}$ and $2^{12} \cdot M_{24}$, respectively, where $M_{22}, M_{23}, M_{24}$ are sporadic Mathieu groups (cf. [ATLAS]). 
In this article, we will describe a general construction of 3-transposition groups as automorphism groups of vertex operator algebras (VOAs). We also try to establish some non-trivial interconnections between the Fischer 3-transposition groups and certain vertex operator subalgebras of the moonshine vertex operator algebra and to ensure that vertex operator algebras are canonical and natural objects for some of sporadic groups in the Monster family. Our methods are similar to that in [HLY12a, HLY12b]. The main idea is to study certain vertex subalgebras generated by the so called Ising vectors (cf. Definition 2.1) and try to analyze the corresponding commutant subalgebras. In particular, we construct two vertex operator algebras $V F_{23}^{\natural}$ and $V F_{22}^{\natural}$ as commutant vertex subalgebras of the famous moonshine VOA $V^{\natural}$. The Fischer 3-transposition group $\mathrm{Fi}_{23}$ (resp. $\mathrm{Fi}_{22}$ ) acts naturally on $V F_{23}^{\natural}$ (resp. $V F_{22}^{\natural}$ ). We also prove the 3-transposition property using the theory of VOA and establish a natural correspondence between the 3-transpositions of $\mathrm{Fi}_{23}\left(\right.$ resp. $\mathrm{Fi}_{22}$ ) and some simple $c=25 / 28$ (resp. $c=11 / 12$ ) Virasoro vectors in $V F_{23}^{\natural}$ (resp. $V F_{22}^{\natural}$ ). We call such a correspondence the Conway-Miyamoto correspondence (see Definition 5.1 for the precise meaning).

The most prominent example of Conway-Miyamoto correspondences is given by the 2A-involutions of the Monster and the Ising vectors of the moonshine VOA. Conway [C85] constructed a slightly modified version of the original Griess algebra [G98a] of dimension $1+196883$ and described a 2A-involution of the Monster in terms of the adjoint action of an idempotent called the transposition axis. Miyamoto [Mi96] showed that Conway's axis corresponds to a $c=1 / 2$ Virasoro vector, called an Ising vector, of $V^{\text {th }}$ and he associated each Ising vector with an involution of $V^{\natural}$ based on the fusion rules of the Virasoro VOA, which may be viewed as a converse of Conway's axis; it turns out that there exists a one-to-one correspondence between the $2 \mathrm{~A}$-elements of the Monster and the Ising vectors of the moonshine VOA (cf. [C85, Mi96, Ma01, H10]). In [HLY12a, HLY12b], some similar correspondences between the 2A-elements of the Baby Monster and $c=7 / 10$ Virasoro vectors of $\sigma$-type of the Baby Monster VOA and that between the 2C-elements of the largest Fischer 3-transposition group and the $c=6 / 7$ Virasoro vectors of $\sigma$-type of the Fischer VOA were established. Both Baby Monster VOA and Fischer VOA are commutant subalgebras of the moonshine VOA and these correspondences are derived from the Monster. Once a correspondence has been established, we can internally describe the corresponding automorphism group based on the VOA structure and study the group as an intrinsic symmetry of the corresponding VOA.

Now let us explain our setting. For a positive integer $n$, let $c_{n}=1-6 /(n+2)(n+3)$ be the central charge of the unitary series of the Virasoro algebra. If a VOA $V$ contains a simple $c=c_{n}$ Virasoro vector $e$, then the linear map $\tau_{e}=(-1)^{4(n+2)(n+3) \mathrm{o}(e)}$ is well-defined, where $\mathrm{o}(e)=e_{(1)}$ is the zero-mode of $e$, and gives rise to an automorphism of $V$ called 
the Miyamoto involution associated to $e$ (cf. [Mi96]). The case $c_{1}=1 / 2$ in $V^{\natural}$ produces a 2 A-element of the Monster. Similarly, one can define another Miyamoto involution $\sigma_{e}$ on a certain subVOA of $V$ using a similar method (see Theorem 2.4 for detail). When $\tau_{e}$ is trivial and $\sigma_{e}$ is well-defined on the whole space $V$, such a Virasoro vector $e$ is said to be of $\sigma$-type on $V$.

A VOA $V$ is said to be of OZ-type (which stands for one-dimensional, zero-dimensional) if $V_{n<0}=0, V_{0}=\mathbb{C} \mathbb{1}$ and $V_{1}=0$. We also assume that there exists a real form $V_{\mathbb{R}}$ of $V$ such that the invariant bilinear form on $V_{\mathbb{R}}$ is positive definite. Our method is to study vertex subalgebras generated by Ising vectors and try to analyze the action of various types of Miyamoto involutions on some commutant subalgebras. In particular, some vertex subalgebras generated by two Ising vectors, which we call dihedral algebras, studied in [LM06, LYY07, LYY05, S07] play some important roles for our study. In [S07], Griess algebras of dihedral subalgebras of a VOA of OZ-type are classified. It turns out that all of them are realizable inside the moonshine VOA (cf. [LM06, LYY07, LYY05]). We will mainly consider the dihedral subalgebras of $2 \mathrm{~A}, 3 \mathrm{~A}$ and $6 \mathrm{~A}$-types, which are simply called the 2A, 3A and 6A-algebras, respectively (cf. Theorem 2.6).

Let $E_{V}$ be the set of Ising vectors of $V_{\mathbb{R}}$. We assume that there exist $a, b \in E_{V}$ such that the dihedral subalgebra $\langle a, b\rangle$ generated by them is isomorphic to the 3Aalgebra. In this case, the subgroup $\left\langle\tau_{a}, \tau_{b}\right\rangle$ is isomorphic to $\mathrm{S}_{3}$ [Mi03, S07]. We set $I_{a, b}=\left\{x \in E_{V} \mid(a \mid x)=(b \mid x)=2^{-5}\right\}$. Then $x \in I_{a, b}$ if and only if both $\langle a, x\rangle$ and $\langle b, x\rangle$ are isomorphic to the $2 \mathrm{~A}$-algebra and each $\tau_{x}, x \in I_{a, b}$, centralizes $\left\langle\tau_{a}, \tau_{b}\right\rangle$ (cf. [Mi96]). We will prove in Corollary 3.6 that the group $G=\left\langle\tau_{x} \mid x \in I_{a, b}\right\rangle$ is a 3-transposition subgroup of $C_{\operatorname{Aut}(V)}\left(\tau_{a}, \tau_{b}\right)$, that is, if $x, y \in I_{a, b}$ then the order of $\tau_{x} \tau_{y}$ is bounded by three. Since $G$ acts trivially on $\langle a, b\rangle$, it is natural to regard $G$ as a subgroup of the automorphism group of the commutant $\operatorname{Com}_{V}\langle a, b\rangle$ of the 3A-subalgebra $\langle a, b\rangle$ in $V$. As an application, we will obtain a realization of $\mathrm{Fi}_{23}$ as an automorphism group of the moonshine VOA. Namely, if we apply Corollary 3.6 to $V^{\natural}$, we obtain the second largest Fischer group $\operatorname{Fi}_{23} \cong C_{\mathbb{M}}\left(\tau_{a}, \tau_{b}\right)$ acting on the commutant of the 3A-algebra $\langle a, b\rangle$ in $V^{\natural}$.

Our construction can be viewed as a sequel of the works in [HLY12a, HLY12b]. In [HLY12b], the $W_{3}$-algebra $W_{3}(4 / 5)$ at $c=4 / 5$ is used to define the Fischer VOA $V F_{24}^{\natural}$ acted by the largest Fischer group $\mathrm{Fi}_{24}$ as the commutant of $W_{3}(4 / 5)$ in the moonshine VOA. The $W_{3}$-algebra $W_{3}(4 / 5)$ is a subalgebra of the $3 \mathrm{~A}$-algebra so that the commutant subalgebra $V F_{23}^{\natural}=\operatorname{Com}_{V^{\natural}}\langle a, b\rangle$ affording the action of $\mathrm{Fi}_{23}$ is a subVOA of the Fischer VOA $V F_{24}^{\natural}$ considered in [HLY12b]. It is known that $\mathrm{Fi}_{24}$ is not a subgroup of the Monster but its extension $3 . \mathrm{Fi}_{24}$ is a subgroup. The group $3 . \mathrm{Fi}_{24}$ is not a 3 -transposition group so that we have to use $\sigma$-involutions, which are well-defined only on the subalgebra $V F_{24}^{\natural}$ of $V^{\natural}$, to realize the 3 -transposition group $\mathrm{Fi}_{24}$ in [HLY12b]. On the other hand, the 3-transposition 
group obtained by Corollary 3.6 is indeed a subgroup of the automorphism group of the whole VOA. Hence, our construction naturally reflects the inclusion $\mathrm{Fi}_{23}<\mathbb{M}=\operatorname{Aut}\left(V^{\natural}\right)$.

In order to study inductive structures and basic sets of the 3-transposition group obtained by Corollary 3.6, we consider the following subalgebras. Let $a, b \in E_{V}$ be a pair such that $\langle a, b\rangle$ is isomorphic to the 3A-algebra and define $I_{a, b}$ as before. We define $X^{[n]}=\left\langle a, b, x^{1}, \ldots, x^{n}\right\rangle$ inductively as follows. We set $X^{[0]}=\langle a, b\rangle$. Suppose we have chosen $x^{1}, \ldots, x^{i} \in I_{a, b}$ and $X^{[i]}=\left\langle a, b, x^{1}, \ldots, x^{i}\right\rangle$ has been defined. We choose $x^{i+1} \in I_{a, b}$ such that $x^{i+1} \notin X^{[i]}$ and $\left(x^{i+1} \mid x^{j}\right)=2^{-5}$ for all $1 \leq j \leq i$ and define $X^{i+1}=\left\langle X^{[i]}, x^{i+1}\right\rangle$ as long as possible. Suppose we have obtained $X^{[n]}=\left\langle a, b, x^{1}, \ldots, x^{n}\right\rangle$ in $V_{\mathbb{R}}$. Set $D^{[0]}=\left\{\tau_{y} \mid y \in I_{a, b}\right\}$ and define $D^{[i]}=\left\langle\tau_{y} \in D^{[i-1]} \mid \tau_{y} \tau_{x^{i}}=\tau_{x^{i}} \tau_{y}\right\rangle$ for $1 \leq i \leq n$. Then $D^{[i]}$ preserves the commutant $\operatorname{Com}_{V} X^{[i]}$ and we obtain the following series of commutant subalgebras acted on by inductive substructures of the 3 -transposition group $G^{[0]}=\left\langle D^{[0]}\right\rangle$.

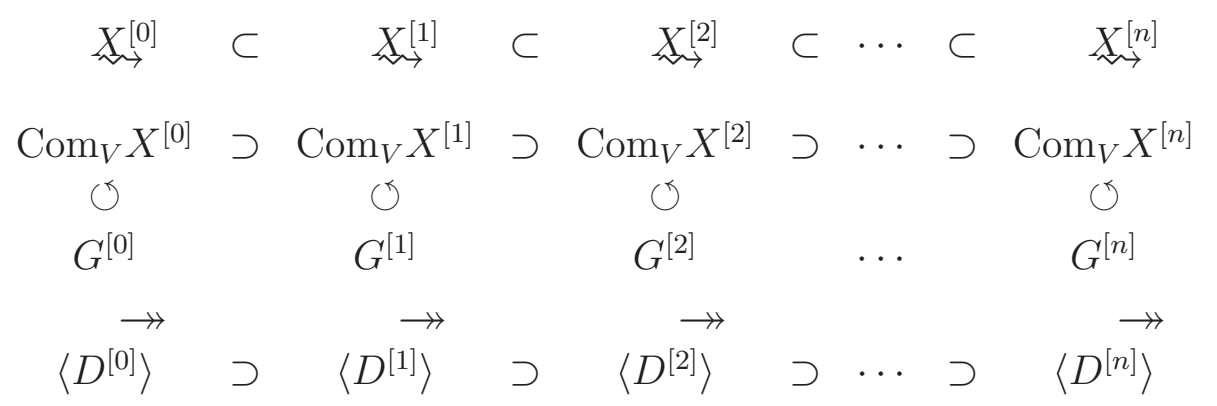

where $G^{[i]}$ is the image of $\left\langle D^{[i]}\right\rangle$ in $\operatorname{Aut}\left(\operatorname{Com}_{V} X^{[i]}\right)$. In the process above, $\left\{\tau_{x^{1}}, \ldots, \tau_{x^{n}}\right\}$ gives a set of mutually commutative involutions in $D^{[0]}$ and $X^{[n]}$ is related to the basic sets of a 3-transposition group $G^{[0]}$. We will prove in Theorem 4.17 that the Griess algebra generated by $a, b, x^{1}, \ldots, x^{n}$ is uniquely determined and $X^{[n]}$ has a full subVOA isomorphic to a tensor product of Virasoro VOAs

$$
L\left(c_{3}, 0\right) \otimes L\left(c_{4}, 0\right) \otimes \cdots \otimes L\left(c_{n+4}, 0\right) .
$$

Our construction of $X^{[n]}$ is compatible with inductive structures of 3-transposition groups in (1.1); thereby applying to the moonshine VOA, we will obtain a series of subVOAs affording the actions of the Fischer 3-transposition groups. As for the moonshine VOA, we obtain $G^{[0]} \cong \mathrm{Fi}_{23}, G^{[1]} \cong \mathrm{Fi}_{22}$ and $G^{[2]} \cong \mathrm{Fi}_{21}=\operatorname{PSU}_{6}(2)$ (cf. Proposition 5.4). We know that there exists a bijection between $I_{a, b} \subset V^{\natural}$ and $D^{[0]} \subset \mathbb{M}$ by the ConwayMiyamoto correspondence of Ising vectors and 2A elements of the Monster. However, if we regard $G^{[i]}$ as a subgroup of $\operatorname{Aut}\left(\mathrm{Com}_{V^{\natural}} X^{[i]}\right)$, the Ising vector $x \in I_{a, b}$ corresponding to $\tau_{x} \in D^{[i]}$ is not contained in the commutant $\operatorname{Com}_{V^{\natural}} X^{[i]}$. Therefore, we need to replace Ising vectors in $V^{\natural}$ by suitable Virasoro vectors in $\operatorname{Com}_{V^{\natural}} X^{[i]}$ for $i=0,1$, which requires some detailed analysis of the subVOAs $X^{[i]}$ and their corresponding Griess algebras. To 
establish the Conway-Miyamoto correspondences for $G^{[0]} \cong \mathrm{Fi}_{23}$ and $G^{[1]} \cong \mathrm{Fi}_{22}$, we also need to determine the invariants of the centralizers $C_{\mathrm{Fi}_{23}}(2 \mathrm{~A})$ and $C_{\mathrm{Fi}_{22}}(2 \mathrm{~A})$ based on explicit calculations on the Griess algebra of $X^{[2]}$. Showing that they are given by $c=c_{5}$ and $c=c_{6}$ Virasoro vectors, we will establish the Conway-Miyamoto correspondences for $\mathrm{Fi}_{23}$ and $\mathrm{Fi}_{22}$ in Theorems 5.8 and 5.14. In principle, we can continue our argument to the next case $\mathrm{Fi}_{21} \cong \mathrm{PSU}_{6}(2)$ where 3 -transpositions of $\mathrm{Fi}_{21}$ are described by $c=c_{7}$ Virasoro vectors. However, by a technical reason, we cannot determine the Griess algebra and its invariant subalgebra of the centralizer (cf. Remark 5.15). The Griess algebra corresponding to $\mathrm{Fi}_{21}$ is rather small and the Conway-Miyamoto correspondences seem to terminate at $\mathrm{Fi}_{22}$ in this series.

The study of $X^{[n]}$ has its own interest since it is related to basic sets of $G^{[0]}$ in (1.1). Since the size of a basic set of $\mathrm{Fi}_{23}$ is 23 , there exist 23 Ising vectors $x^{1}, \ldots, x^{23} \in I_{a, b} \subset$ $V^{\natural}$ satisfying our conditions and there is a subalgebra $X^{[23]}=\left\langle a, b, x^{1}, \ldots, x^{23}\right\rangle$ in $V^{\natural}$. Surprisingly, the central charge of the Virasoro frame in (1.2) is equal to 24 when $n=23$ and hence $X^{[23]}$ is a full subVOA of $V^{\natural}$. Therefore, it is possible to analyze $V^{\natural}$ as a finite extension of $X^{[23]}$. We will discuss a construction of $V^{\natural}$ starting from $X^{[23]}$ in [CLY].

The organization of this article is as follows. In Section 2, we recall some basic properties about Virasoro VOAs [FZ92, W93] and the dihedral algebras constructed in [LYY07, LYY05]. In Section 3, we give a construction of 3-transposition groups using the 3A-algebra. We first fix a pair of Ising vectors $a, b \in V$ such that $a$ and $b$ generate a 3A-algebra in $V$ and then consider the set of Ising vectors $I_{a, b}$ of $V$ such that $\langle a, x\rangle$ and $\langle b, x\rangle$ are isomorphic to the 2A-algebra for all $x \in I_{a, b}$. We will show that the Miyamoto involutions associated to Ising vectors of $I_{a, b}$ generate a 3 -transposition group in Aut $(V)$. In Section 4, we will determine all possible structures of the subVOA generated by $a, b, x$, $y$ for any $x, y \in I_{a, b}$ and then introduce inductive subalgebras $X^{[i]}=\left\langle a, b, x^{1}, \ldots, x^{i}\right\rangle$ for $i \geq 0$. The commutants of subalgebras $X^{[i]}$ are used to study inductive structures of the 3 -transposition group obtained in Section 3. The results in Section 4.2 will not be used in the later section but we will need these results in our future work so that we include them. In Section 5, we will apply our results to the moonshine VOA and the Fischer 3transposition groups $\mathrm{Fi}_{23}$ and $\mathrm{Fi}_{22}$. The Conway-Miyamoto correspondence between the 2A-involutions of $\mathrm{Fi}_{23}$ and $c=c_{5}$ Virasoro vectors of $V F_{23}^{\natural}=\operatorname{Com}_{V^{\natural}} X^{[0]}$ and that between the 2A-involutions of $\mathrm{Fi}_{22}$ and $c=c_{6}$ Virasoro vectors of $\sigma$-type of $V F_{22}^{\natural}=\operatorname{Com}_{V^{\natural}} X^{[1]}$ will be established. In the appendix, we will give explicit constructions of the VOAs studied in Section 4.

The authors used computer algebra systems Risa/Asir for the calculations in Griess algebras and GAP 4.7.4 for Linux for the character calculations of finite groups. 
Acknowledgement. Part of this work has been done while the author(s) were staying at Academia Sinica in September 2011, March 2013 and March 2015, at National Taitung University in March 2013 and March 2015, at Imperial College London during "Majorana Theory, the Monster and Beyond" in September 2013, at National Dong Hwa University in March 2014, at Mathematisches Forschungsinstitut Oberwolfach during "Subfactor and Conformal Field Theory" in March 2015, and at Sichuan University in September 2015. They gratefully acknowledge the hospitality there. H.Y. thanks Masaaki Kitazume, Atsushi Matsuo, Masahiko Miyamoto, Hiroki Shimakura and Katsushi Waki for valuable comments and helpful discussions.

Notation and terminology. In this paper, vertex operator algebras (VOAs) are defined over the complex number field $\mathbb{C}$. A VOA $V$ is called of OZ-type if it has the $L(0)$-grading $V=\oplus_{n \geq 0} V_{n}$ such that $V_{0}=\mathbb{C} \mathbb{1}$ and $V_{1}=0$. In this case, $V$ is equipped with a unique invariant bilinear form such that $(\mathbb{1} \mid \mathbb{1})=1$. A real form $V_{\mathbb{R}}$ of $V$ is called compact if the associated bilinear form is positive definite. We will mainly consider a VOA of OZ-type having a compact real form. For a subset $A$ of $V$, the vertex subalgebra generated by $A$ is denoted by $\langle A\rangle$. (In most cases, $\langle A\rangle$ indeed becomes a subVOA in this paper.) For $a \in V_{n}$ we $\operatorname{define} \operatorname{wt}(a)=n$. We write $Y(a, z)=\sum_{n \in \mathbb{Z}} a_{(n)} z^{-n-1}$ for $a \in V$ and define its zero-mode by $\mathrm{o}(a):=a_{(\mathrm{wt}(a)-1)}$ if $a$ is homogeneous and extend linearly. The weight two subspace $V_{2}$ carries a structure of a commutative algebra defined by the product $\mathrm{o}(a) b=a_{(1)} b$ for $a, b \in V_{2}$. This algebra is called the Griess algebra of $V$. A Virasoro vector is $a \in V_{2}$ such that $a_{(1)} a=2 a$. The subalgebra $\langle a\rangle$ is isomorphic to a Virasoro VOA with central charge $2(a \mid a)$. If two Virasoro vectors $a$ and $b$ are orthogonal, we will denote their sum by $a \dot{+} b$. We denote by $L(c, h)$ the irreducible highest weight module over the Virasoro algebra with central charge $c$ and highest weight $h$. A simple $c=c_{e}$ Virasoro vector $e \in V$ is is a Virasoro vector such that $\langle e\rangle \cong L\left(c_{e}, 0\right)$. If $e$ is taken from a compact real form of $V$ then $e$ is always simple. A Virasoro vector $\omega$ is called the conformal vector of $V$ if each graded subspace $V_{n}$ agrees with $\operatorname{Ker}_{V}(\mathrm{o}(\omega)-n)$ and satisfies $\omega_{(0)} a=a_{(-2)} \mathbb{1}$ for all $a \in V$. The half of the conformal vector gives the unit of the Griess algebra and hence uniquely determined. We write $\omega_{(n+1)}=L(n)$ for $n \in \mathbb{Z}$. A Virasoro vector $e$ of $V$ is called characteristic if it is fixed by $\operatorname{Aut}(V)$. Clearly the conformal vector is characteristic in $V$. A $\operatorname{subVOA}(W, e)$ of $V$ is a pair of a subalgebra $W$ of $V$ together with a Virasoro vector $e$ in $W$ such that $e$ is the conformal vector of $W$. We often omit $e$ and simply denote by $W$. The commutant subalgebra of $(W, e)$ in $V$ is defined by $\operatorname{Com}_{V} W:=\operatorname{Ker}_{V} e_{(0)}$ (cf. [FZ92]). A subVOA $W$ of $V$ is called full if $V$ and $W$ shares the same conformal vector. For a subgroup $G$ of $\operatorname{Aut}(V)$, we denote the set of $G$-invariants by $V^{G}$. 


\section{The dihedral subalgebras}

First we recall some basic properties about Virasoro VOAs [FZ92, W93] and the dihedral algebras constructed in [LYY07, LYY05].

\subsection{Virasoro vertex operator algebras}

Let

$$
\begin{aligned}
c_{n} & :=1-\frac{6}{(n+2)(n+3)}, \quad n=1,2,3, \ldots, \\
h_{r, s}^{(n)} & :=\frac{\{r(n+3)-s(n+2)\}^{2}-1}{4(n+2)(n+3)}, \quad 1 \leq r \leq n+1, \quad 1 \leq s \leq n+2 .
\end{aligned}
$$

It is shown in [W93] that $L\left(c_{n}, 0\right)$ is rational and $L\left(c_{n}, h_{r, s}^{(n)}\right), 1 \leq s \leq r \leq n+1$, are all the irreducible $L\left(c_{n}, 0\right)$-modules (see also [DMZ94]). This is the so-called unitary series of the Virasoro VOAs. Note that $h_{r, s}^{(n)}=h_{n+2-r, n+3-s}^{(n)}$. The fusion rules among $L\left(c_{n}, 0\right)$-modules are computed in [W93] and given by

$$
L\left(c_{n}, h_{r_{1}, s_{1}}^{(n)}\right) \otimes L\left(c_{n}, h_{r_{2}, s_{2}}^{(n)}\right)=\sum_{\substack{1 \leq i \leq M \\ 1 \leq j \leq N}} L\left(c_{n}, h_{\left|r_{1}-r_{2}\right|+2 i-1,\left|s_{1}-s_{2}\right|+2 j-1}^{(n)}\right),
$$

where $M=\min \left\{r_{1}, r_{2}, n+2-r_{1}, n+2-r_{2}\right\}$ and $N=\min \left\{s_{1}, s_{2}, n+3-s_{1}, n+3-s_{2}\right\}$.

Definition 2.1. A Virasoro vector $e$ with central charge $c$ is called simple if $\langle e\rangle \cong L(c, 0)$. A simple $c=1 / 2$ Virasoro vector is called an Ising vector.

The fusion rules among $L\left(c_{m}, 0\right)$-modules have a canonical $\mathbb{Z}_{2}$-symmetry.

Theorem 2.2 ([Mi96]). Let $V$ be a VOA and $e \in V$ a simple Virasoro vector with central charge $c_{n}$. Denote by $V_{e}\left[h_{r, s}^{(n)}\right]$ the sum of irreducible $\langle e\rangle \cong L\left(c_{n}, 0\right)$-submodules isomorphic to $L\left(c_{n}, h_{r, s}^{(n)}\right), 1 \leq s \leq r \leq n+1$. Then the linear map

$$
\tau_{e}:=(-1)^{4(n+2)(n+3) \circ(e)}=\left\{\begin{array}{lll}
(-1)^{r+1} & \text { on } V_{e}\left[h_{r, s}^{(n)}\right] & \text { if } n \text { is even }, \\
(-1)^{s+1} & \text { on } V_{e}\left[h_{r, s}^{(n)}\right] & \text { if } n \text { is odd },
\end{array}\right.
$$

defines an automorphism of $V$.

The automorphism $\tau_{e}$ is called the Miyamoto involution associated to $e$.

Definition 2.3. Let $e$ be a simple $c=c_{n}$ Virasoro vector of $V$. Set

$$
P_{n}:= \begin{cases}\left\{h_{1, s}^{(n)} \mid 1 \leq s \leq n+2\right\} & \text { if } n \text { is even } \\ \left\{h_{r, 1}^{(n)} \mid 1 \leq r \leq n+1\right\} & \text { if } n \text { is odd }\end{cases}
$$


It follows from the fusion rules in (2.3) that the subspace

$$
V_{e}\left[P_{n}\right]=\bigoplus_{h \in P_{n}} V_{e}[h]
$$

forms a subalgebra of $V$. We say that $e$ is of $\sigma$-type on $V$ if $V=V_{e}\left[P_{n}\right]$.

Theorem 2.4 ([Mi96]). The linear map

$$
\sigma_{e}:=\left\{\begin{array}{lll}
(-1)^{s+1} & \text { on } V_{e}\left[h_{1, s}^{(n)}\right] & \text { if } n \text { is even } \\
(-1)^{r+1} & \text { on } V_{e}\left[h_{r, 1}^{(n)}\right] & \text { if } n \text { is odd }
\end{array}\right.
$$

defines an element of $\operatorname{Aut}\left(V_{e}\left[P_{n}\right]\right)$.

The automorphism $\sigma_{e}$ is called a Miyamoto involution of $\sigma$-type or simply a $\sigma$ involution. Suppose $e$ is an Ising vector, i.e., simple $c=1 / 2$ Virasoro vector. Then we have

$$
\tau_{e}=\left\{\begin{array}{cll}
1 & \text { on } & V_{e}[0] \oplus V_{e}[1 / 2] \\
-1 & \text { on } & V_{e}[1 / 16]
\end{array}\right.
$$

If $e$ is of $\sigma$-type on $V$, the linear map $\sigma_{e}$ is defined by

$$
\sigma_{e}:=\left\{\begin{array}{ccc}
1 & \text { on } & V_{e}[0] \\
-1 & \text { on } & V_{e}[1 / 2]
\end{array}\right.
$$

Alternatively, one can define $\sigma_{e}=(-1)^{20(e)}$. By the definition of Miyamoto involutions, we have the following conjugation.

Proposition 2.5. Let e be a simple $c=c_{n}$ Virasoro vector of $V$. Then $\tau_{g e}=g \tau_{e} g^{-1}$ for any $g \in \operatorname{Aut}(V)$. If e is of $\sigma$-type on $V$, we have $\sigma_{g e}=g \sigma_{e} g^{-1}$.

For an Ising vector $e$ and $v \in V_{2}$, one has $v+\tau_{e} v \in V^{\left\langle\tau_{e}\right\rangle}$ and hence $\sigma_{e}\left(v+\tau_{e} v\right)$ is a well-defined element in $V_{2}$. Moreover, we have (cf. Eq. (2.2) of [S07]):

$$
e_{(1)} v=8(e \mid v) e+\frac{5}{32} v+\frac{3}{32} \tau_{e} v-\frac{1}{8} \sigma_{e}\left(v+\tau_{e} v\right)
$$

In particular, if $\tau_{e} v=v$, we have

$$
\sigma_{e} v=v+32(e \mid v) e-4 e_{(1)} v
$$

These relations will be used in Section 3.

In [LYY07, LYY05], subalgebras generated by two Ising vectors are constructed using the $E_{8}$-lattice and such subalgebras are classified in [S07] at the level of Griess algebras. 
Theorem 2.6 ([S07]). Let $V$ be a VOA of OZ-type with compact real form $V_{\mathbb{R}}$ and let $e$ and $f$ be distinct Ising vectors of $V_{\mathbb{R}}$. Then the Griess algebra of the subalgebra $\langle e, f\rangle$ is isomorphic to one of the eight algebras called 2A, 3A, 4A, 5A, 6A, 4B, $2 B$ and $3 C$ type constructed in [LYYO\%, LYY05]. The inner product and the dimension of the Griess algebra are as follows ${ }^{1}$.

\begin{tabular}{c|ccccccccc} 
Type & $1 \mathrm{~A}$ & $2 \mathrm{~A}$ & $3 \mathrm{~A}$ & $4 \mathrm{~A}$ & $5 \mathrm{~A}$ & $6 \mathrm{~A}$ & $4 \mathrm{~B}$ & $2 \mathrm{~B}$ & $3 \mathrm{C}$ \\
\hline $2^{10}(e \mid f)$ & $2^{8}$ & $2^{5}$ & 13 & 8 & 6 & 5 & 4 & 0 & 4 \\
\hline $\operatorname{dim}\langle e, f\rangle_{2}$ & 1 & 3 & 4 & 5 & 6 & 8 & 5 & 2 & 3 \\
\hline \# of Ising vectors & 1 & 3 & 3 & 4 & 5 & 7 & 5 & 2 & 3
\end{tabular}

We call $\langle e, f\rangle$ the dihedral subalgebra and we denote the dihedral subalgebra of type $n \mathrm{X}$ by $U_{n \mathrm{X}}$. As a by-product of the classification of dihedral subalgebras, the following 6-transposition property was established in [S07].

Theorem 2.7 ([S07]). Let $V$ be a VOA of OZ-type with compact real form $V_{\mathbb{R}}$ and let $e$ and $f$ be distinct Ising vectors of $V_{\mathbb{R}}$. Then

(1) If $\langle e, f\rangle$ is $2 A$ or $2 B$-type then $\left|\tau_{e} \tau_{f}\right|$ divides 2.

(2) If $\langle e, f\rangle$ is $3 A$ or $3 C$-type then $\left|\tau_{e} \tau_{f}\right|=3$.

(3) If $\langle e, f\rangle$ is $4 A$ or $4 B$-type then $\left|\tau_{e} \tau_{f}\right|=2$ or 4 .

(4) If $\langle e, f\rangle$ is 5A-type then $\left|\tau_{e} \tau_{f}\right|=5$.

(5) If $\langle e, f\rangle$ is 6A-type then $\left|\tau_{e} \tau_{f}\right|=3$ or 6.

(6) $\tau_{e} f=f$ if and only if $\langle e, f\rangle$ is either $2 A$ or $2 B$-type.

(7) $\tau_{e} f=\tau_{f} e$ if and only if $\langle e, f\rangle$ is either $3 A$ or 3C-type.

In particular, the order of $\tau_{e} \tau_{f}$ is bounded by 6 .

The next lemma also follows from the classification of the dihedral algebras.

Lemma 2.8. Let $a^{1}, a^{2}, b^{1}, b^{2}$ be Ising vectors and set $U^{1}=\left\langle a^{1}, b^{1}\right\rangle$ and $U^{2}=\left\langle a^{2}, b^{2}\right\rangle$. If $U^{1}$ is a proper subalgebra of $U^{2}$ then the types of $\left(U^{1}, U^{2}\right)$ are either $(2 \mathrm{~A}, 4 \mathrm{~B}),(2 \mathrm{~A}, 6 \mathrm{~A})$, $(2 \mathrm{~B}, 4 \mathrm{~A})$ or $(3 \mathrm{~A}, 6 \mathrm{~A})$.

In the rest of this section, we will summarize some properties of the dihedral subalgebras of 2A, 3A and 6A-types which will be used in the later sections. See [LYY05] for details.

\section{$2.2 \quad 2$ A-algebra}

It follows from Theorem 2.6 that $\langle a, b\rangle$ is of 2A-type if and only if $(a \mid b)=2^{-5}$. Let $U_{2 \mathrm{~A}}=\langle a, b\rangle$ be the 2A-algebra. Then $a$ and $b$ are of $\sigma$-type on $\langle a, b\rangle$ and satisfies $\sigma_{a} b=\sigma_{b} a$.

\footnotetext{
${ }^{1}$ Here we include the case $e=f$ which is called the 1A-type.
} 
Set $a \circ b:=\sigma_{a} b=\sigma_{b} a$. It follows from (2.6) that

$$
a \circ b=a+b-4 a_{(1)} b .
$$

Theorem 2.9. Let $U_{2 \mathrm{~A}}=\langle a, b\rangle$ be the 2A-algebra.

(1) There are exactly three Ising vectors in of $U_{2 \mathrm{~A}}$, namely, $a, b$ and $a \circ b$.

(2) The Griess algebra of $U_{2 \mathrm{~A}}$ is 3-dimensional spanned by these three Ising vectors.

(3) $(a \mid b)=(a \mid a \circ b)=(b \mid a \circ b)=2^{-5}$.

(4) $\operatorname{Aut}\left(U_{2 \mathrm{~A}}\right)=\left\langle\sigma_{a}, \sigma_{b}\right\rangle \cong \mathrm{S}_{3}$.

(5) Suppose $U_{2 \mathrm{~A}}=\langle a, b\rangle$ is a subalgebra of a VOA V. Then $\tau_{a \circ b}=\tau_{a} \tau_{b}=\tau_{b} \tau_{a}$ on $V$.

We will call the set of Ising vectors $\{a, b, a \circ b\}$ of $\langle a, b\rangle$ a $2 A$-triple. The subalgebra generated by three Ising vectors of $\sigma$-type was classified in [Ma05] and the following "No 2A-tetrahedron lemma" holds (cf. Proposition 1 of $[\mathrm{Ma05}]^{2}$ ).

Lemma 2.10. Let $a, b, c$ be Ising vectors such that $(a \mid b)=(a \mid c)=(b \mid c)=2^{-5}$ and $c \notin\langle a, b\rangle$. Then we have $(a \circ b \mid c)=(a \mid b \circ c)=(b \mid a \circ c)=0$.

\section{$2.3 \quad 3$ A-algebra}

It follows from Theorem 2.6 that $\langle a, b\rangle$ is of 3A-type if and only if $(a \mid b)=13 \cdot 2^{-10}$. Let $U_{3 \mathrm{~A}}=\langle a, b\rangle$ be the 3A-algebra. Then $\tau_{a} b=\tau_{b} a$ holds. Set $c=\tau_{a} b$ and define

$$
u=u_{a, b}=\frac{2^{6}}{135}\left(2 a+2 b+c-16 a_{(1)} b\right) .
$$

Then $u$ is a $c=4 / 5$ Virasoro vector in $U_{3 \mathrm{~A}}$.

Theorem 2.11. Let $U_{3 \mathrm{~A}}=\langle a, b\rangle$ be the 3 A-algebra.

(1) There are exactly three Ising vectors in $U_{3 \mathrm{~A}}$, namely, $a, b$ and $c$.

(2) $\operatorname{Aut}\left(U_{3 \mathrm{~A}}\right)=\left\langle\tau_{a}, \tau_{b}\right\rangle \cong \mathrm{S}_{3}$.

(3) The Griess algebra of $U_{3 \mathrm{~A}}$ is 4-dimensional and spanned by $u, a, b$ and $c$.

(4) The $c=4 / 5$ Virasoro vector $u$ is characteristic in $U_{3 \mathrm{~A}}$.

(5) The multiplications and inner products of the Griess algebra are as follows.

$$
\begin{aligned}
& a_{(1)} b=-\frac{135}{2^{10}} u+\frac{1}{2^{3}} a+\frac{1}{2^{3}} b+\frac{1}{2^{4}} c, \quad a_{(1)} u=\frac{5}{2^{4}} u+\frac{4}{3^{2}} a-\frac{2}{3^{2}} b-\frac{2}{3^{2}} c, \\
& (a \mid b)=(a \mid c)=(b \mid c)=\frac{13}{2^{10}}, \quad(a \mid u)=(b \mid u)=(c \mid u)=\frac{1}{2^{4}} .
\end{aligned}
$$

(6) There is a unique characteristic $c=6 / 7$ Virasoro vector in $\langle a, b\rangle$, which is given by

$$
v=-\frac{5}{14} u+\frac{16}{21}(a+b+c) .
$$

Moreover, $u$ and $v$ are mutually orthogonal and $u+v$ is the conformal vector of $\langle a, b\rangle$.

\footnotetext{
${ }^{2}$ Proposition 3.3.8 of arXiv:math/0311400.
} 
We call the set of Ising vectors in $U_{3 \mathrm{~A}}$ a $3 A$-triple. We will need the following relations later.

Lemma 2.12. In the 3A-algebra, the following holds.

$$
\sigma_{a} u=-\frac{1}{2^{2}} u+\frac{2}{3^{2}} a+\frac{8}{3^{2}}(b+c), \quad \sigma_{a}(b+c)=\frac{135}{2^{7}} u-\frac{3}{2^{4}} a+\frac{1}{2^{2}}(b+c) .
$$

\subsection{A-algebra}

It follows from Theorem 2.6 that $\langle a, b\rangle$ is of 6A-type if and only if $(a \mid b)=5 \cdot 2^{-10}$. Let $U_{6 \mathrm{~A}}=\langle a, b\rangle$ be the $6 \mathrm{~A}$-algebra. There are 7 Ising vectors in the $6 \mathrm{~A}$-algebra. Namely, $a$, $b, \tau_{a} b, \tau_{b} a, \tau_{a} \tau_{b} a, \tau_{b} \tau_{a} b$ and

$$
x=a \circ \tau_{b} \tau_{a} b=b \circ \tau_{a} \tau_{b} a=\tau_{b} a \circ \tau_{a} b .
$$

The Ising vector $x$ is of $\sigma$-type and defines a $\sigma$-involution on $\langle a, b\rangle$. Inside the 6A-algebra, there are two 3A-subalgebras and the 3-sets $\left\{a, \tau_{b} a, \tau_{a} \tau_{b} a\right\}$ and $\left\{b, \tau_{a} b, \tau_{b} \tau_{a} b\right\}$ form the 3A-triples with the same characteristic $c=4 / 5$ Virasoro vector $u_{a, \tau_{b} a}=u_{b, \tau_{a} b}$. The automorphism $\tau_{a} \tau_{b}$ acts as a 3-cycle on each of the 3A-triples and has order 3 on $\langle a, b\rangle$.

Theorem 2.13. Let $U_{6 \mathrm{~A}}=\langle a, b\rangle$ be the $6 A$-algebra.

(1) The set of Ising vectors of $U_{6 \mathrm{~A}}$ is given by $\left\{x, a, b, \tau_{a} b, \tau_{b} a, \tau_{a} \tau_{b} a, \tau_{b} \tau_{a} b\right\}$.

(2) $x$ is the unique characteristic $\sigma$-type Ising vector in $U_{6 \mathrm{~A}}$ and $\sigma_{x}$ is central in $\operatorname{Aut}\left(U_{6 \mathrm{~A}}\right)$.

(3) $\left\{x, a, \tau_{b} \tau_{a} b\right\},\left\{x, b, \tau_{a} \tau_{b} a\right\}$ and $\left\{x, \tau_{b} a, \tau_{a} b\right\}$ form $2 A$-triples in $\langle a, b\rangle$.

(4) $\left\langle a, \tau_{b} a, \tau_{a} \tau_{b} a\right\rangle$ and $\left\langle b, \tau_{a} b, \tau_{b} \tau_{a} b\right\rangle$ are isomorphic to the $3 A$-algebra with the same characteristic $c=4 / 5$ Virasoro vector $u=u_{a, \tau_{b} a}=u_{b, \tau_{a} b}$.

(5) The Griess algebra of $U_{6 \mathrm{~A}}$ is 8-dimensional with a basis $\left\{u, x, a, b, \tau_{a} b, \tau_{b} a, \tau_{a} \tau_{b} a, \tau_{b} \tau_{a} b\right\}$.

(6) $\operatorname{Aut}\left(U_{6 \mathrm{~A}}\right)=\left\langle\tau_{a} \sigma_{x}, \tau_{b}\right\rangle$ is isomorphic to the dihedral group of order 12.

(7) Set $\rho=\tau_{a} \tau_{b} \sigma_{x}$ and $e^{i}=\rho^{i} a$. Then $\left\{a, b, \tau_{a} b, \tau_{b} a, \tau_{a} \tau_{b} a, \tau_{b} \tau_{a} b\right\}=\left\{e^{i} \mid 0 \leq i \leq 5\right\}$ and the multiplications and inner products of the Griess algebra are as follows.

$$
\begin{aligned}
& u_{(1)} x=0, \quad u_{(1)} e^{i}=-\frac{135}{2^{10}} u+\frac{4}{3^{2}} e^{i}-\frac{2}{3^{2}}\left(e^{i+2}+e^{i+4}\right), \quad(u \mid x)=0, \quad\left(u \mid e^{i}\right)=\frac{1}{2^{4}}, \\
& x_{(1)} e^{i}=\frac{1}{4} x+e^{i}-e^{i+3}, \quad e_{(1)}^{0} e^{1}=\frac{45}{2^{10}} u+\frac{1}{2^{5}}\left(x+e^{0}+e^{1}-e^{2}-e^{3}-e^{4}-e^{5}\right), \\
& \left(x \mid e^{i}\right)=\frac{1}{2^{5}}, \quad\left(e^{i} \mid e^{i+1}\right)=\frac{5}{2^{10}}, \quad\left(e^{i} \mid e^{i+2}\right)=\frac{13}{2^{10}}, \quad\left(e^{i} \mid e^{i+3}\right)=\frac{1}{2^{5}} .
\end{aligned}
$$

(8) The commutant of $\left\langle a, \tau_{b} a\right\rangle$ in $\langle a, b\rangle$ is generated by a $c=25 / 28$ Virasoro vector

$$
f=-\frac{15}{56} u+\frac{1}{2} x-\frac{2}{21}\left(a+\tau_{b} a+\tau_{a} \tau_{b} a\right)+\frac{2}{3}\left(b+\tau_{a} b+\tau_{b} \tau_{a} b\right) .
$$

Therefore, $\langle a, b\rangle$ has a unitary Virasoro frame isomorphic to $L(4 / 5,0) \otimes L(6 / 7,0) \otimes L(25 / 28,0)$. 
The characteristic Ising vector $x$ is called central since $\sigma_{x}$ is central in the automorphism group of the 6A-algebra. We collect some technical relations needed in the later arguments.

Lemma 2.14. Let $\langle a, b\rangle$ be the $6 A$-algebra and let $u$ be the characteristic $c=4 / 5$ Virasoro vector and $x$ the central Ising vector. Then one has

$$
\begin{aligned}
& a_{(1)}\left(\tau_{b} a\right)_{(1)} x=-\frac{45}{2^{10}} u+\frac{1}{2^{7}}\left(7 x+11 a+b+5 \tau_{b} a-7 \tau_{b} \tau_{a} b+3 \tau_{a} \tau_{b} a-\tau_{a} b\right), \\
& \sigma_{a}\left(b+\tau_{a} b\right)=-\frac{45}{2^{7}} u_{a, b}-\frac{1}{2^{2}} x+\frac{1}{2^{4}} a+\frac{1}{2^{2}} \tau_{b} \tau_{a} b+b+\tau_{a} b+\frac{1}{2^{2}}\left(\tau_{b} a+\tau_{a} \tau_{b} a\right) .
\end{aligned}
$$

Remark 2.15. In some situation, it is useful to display the relations above using another labeling. We rename the Ising vectors so that $a$ and $b$ generate a $3 A$-algebra and $x$ is the central Ising vector in a $6 \mathrm{~A}$-algebra. Let $c=\tau_{a} b=\tau_{b} a$. Then $\{a, b, c\}$ forms a $3 \mathrm{~A}$-triple and $\{a \circ x, b \circ x, c \circ x\}$ gives another 3A-triple. Lemma 2.14 can be rewritten as follows.

$$
\begin{aligned}
& a_{(1)} b_{(1)} x=-\frac{45}{2^{10}} u_{a, b}+\frac{1}{2^{7}}(7 x+11 a+5 b+3 c-7 a \circ x-b \circ x+c \circ x), \\
& \sigma_{a}(b \circ x+c \circ x)=-\frac{45}{2^{7}} u-\frac{1}{2^{2}} x+\frac{1}{2^{4}} a+\frac{1}{2^{2}} a \circ x+\frac{1}{2^{2}}(b+c)+b \circ x+c \circ x .
\end{aligned}
$$

\section{3-transposition property}

Suppose that $V$ has a grading $V=\bigoplus_{n \geq 0} V_{n}$ with $V_{0}=\mathbb{C} \mathbb{1}$ and $V_{1}=0$. We also assume that $V$ has a compact real form $V_{\mathbb{R}}$ and all Ising vectors are taken from it.

Lemma 3.1. Let $e$ be an Ising vector and $v \in V_{2}$. Then $\tau_{e} v \in\langle e, v\rangle$. If $v \in V^{\left\langle\tau_{e}\right\rangle}$, then $\sigma_{e} v \in\langle e, v\rangle$.

Proof: We have the eigenspace decomposition

$$
\left.V_{2}=\mathbb{C} e \oplus\left(V_{2}\right)_{e}[0] \oplus\left(V_{2}\right)_{e} e^{[1 / 2]} \oplus\left(V_{2}\right)_{e} e^{[1 / 16}\right] .
$$

Decompose $v=\lambda e+v_{0}+v_{1 / 2}+v_{1 / 16}$ with $\lambda \in \mathbb{C}$ and $v_{h} \in\left(V_{2}\right)_{e}[h]$. Then we have

$$
16 e_{(1)} v=32 \lambda e+8 v_{1 / 2}+v_{1 / 16} \text { and } 256 e_{(1)} e_{(1)} v=1024 \lambda e+64 v_{1 / 2}+v_{1 / 16} .
$$

It follows that all of $v_{0}, v_{1 / 2}, v_{1 / 16}$ are in $\operatorname{Span}\left\{v, e, e_{(1)} v, e_{(1)} e_{(1)} v\right\}$. Therefore, $\tau_{e} v=$ $v-2 v_{1 / 16} \in\langle e, v\rangle$. If $v \in V^{\left\langle\tau_{e}\right\rangle}$ then it follows from (2.6) that $\sigma_{e} v \in\langle e, v\rangle$.

Lemma 3.2. Let $a, b, x$ be Ising vectors of $V_{\mathbb{R}}$ such that $\tau_{a} b=\tau_{b} a$ and $\langle a, x\rangle \cong\langle b, x\rangle \cong$ $U_{2 \mathrm{~A}}$. Then $\langle a, b\rangle \cong U_{3 \mathrm{~A}},\langle a, b, x\rangle \cong U_{6 \mathrm{~A}}$ and $x$ is the central Ising vector of $\langle a, b, x\rangle$. 
Proof: Since $a, b \in V^{\left\langle\tau_{x}\right\rangle}, x$ is of $\sigma$-type on $\langle a, b, x\rangle$ and one has $\tau_{a \circ x}=\tau_{a} \tau_{x}$. We will show that $\langle a \circ x, b\rangle=\langle a, b, x\rangle$. By Lemma 3.1, $\langle a \circ x, b\rangle \subset\langle a, b, x\rangle$. Since $\tau_{x} b=b$, we have $\tau_{a \circ x} b=\tau_{a} \tau_{x} b=\tau_{a} b$. By our assumption, we have $\tau_{a} b=\tau_{b} a$. Then $\tau_{a \circ x} b=\tau_{a} \tau_{x} b=$ $\tau_{a} b=\tau_{b} a$ and $a=\tau_{b} \tau_{a \circ x} b \in\langle a \circ x, b\rangle$ by Lemma 3.1. Since $a \circ(a \circ x)=x$, we also have $x=a+a \circ x-4 a_{(1)}(a \circ x) \in\langle a \circ x, b\rangle$. Therefore, $a, b, x \in\langle a \circ x, b\rangle$ and $\langle a, b, x\rangle=\langle a \circ x, b\rangle$. By (7) of Theorem 2.7, the assumption $\tau_{a} b=\tau_{b} a$ implies $\langle a, b\rangle$ is isomorphic to either $U_{3 \mathrm{~A}}$ or $U_{3 \mathrm{C}}$. Since $\langle a, b\rangle \subset\langle a \circ x, b\rangle$, the possible types of $\langle a, b\rangle$ and $\langle a \circ x, b\rangle$ are only $U_{3 \mathrm{~A}}$ and $U_{6 \mathrm{~A}}$, respectively, by Lemma 2.8. As is of $\sigma$-type on $\langle a, b, x\rangle \cong U_{6 \mathrm{~A}}, x$ is the unique central Ising vector in $\langle a, b, x\rangle$. This completes the proof.

Lemma 3.3. Let $a, b, x$ be Ising vectors of $V_{\mathbb{R}}$ and such that $\langle a, x\rangle \cong\langle b, x\rangle \cong U_{2 \mathrm{~A}}$ and $\langle a, b\rangle \cong U_{6 \mathrm{~A}}$. Let $z$ be the central Ising vector of $\langle a, b\rangle$. Then $\tau_{x}=\tau_{z}$ on $V_{\mathbb{R}}$.

Proof: Suppose $\tau_{x} \neq \tau_{z}$ on $V_{\mathbb{R}}$. Let $a^{\prime}=\tau_{b} \tau_{a} b$. Then $a, a^{\prime}$ and $z=a \circ a^{\prime}$ form a $2 \mathrm{~A}$-triple by (3) of Theorem 2.13. By Proposition 2.5 and (5) of Theorem 2.9, we have

$$
\tau_{z}=\tau_{a} \tau_{a^{\prime}}=\tau_{a} \tau_{\tau_{b} \tau_{a} b}=\left(\tau_{a} \tau_{b}\right)^{3}
$$

Since $\langle a, x\rangle$ and $\langle b, x\rangle$ are of 2A-type, we have $\left[\tau_{x}, \tau_{a}\right]=\left[\tau_{x}, \tau_{b}\right]=1$. Then $\tau_{x}$ commutes with $\tau_{z}=\left(\tau_{a} \tau_{b}\right)^{3}$ and $\tau_{a^{\prime}}=\tau_{a} \tau_{z}$. Therefore, $G=\left\langle\tau_{a}, \tau_{a^{\prime}}, \tau_{x}\right\rangle \subset \operatorname{Aut}\left(V_{\mathbb{R}}\right)$ is an abelian subgroup. The order of $\tau_{a} \tau_{b}$ is either 3 or 6 on $V_{\mathbb{R}}$. Since

$$
\left(\tau_{a \circ x} \tau_{b}\right)^{3}=\left(\tau_{x} \tau_{a} \tau_{b}\right)^{3}=\tau_{x}^{3}\left(\tau_{a} \tau_{b}\right)^{3}=\tau_{x} \tau_{z} \neq 1
$$

the order of $\tau_{a \circ x} \tau_{b}$ is 6 by the 6 -transposition property and we have $\langle a \circ x, b\rangle \cong U_{6 \mathrm{~A}}$ by Theorem 2.7. It follows from (3) of Theorem 2.13 that $a \circ x$ and $\tau_{b} \tau_{a \circ x} b$ generates a 2A-subalgebra in $\langle a \circ x, b\rangle$. On the other hand, by (5) of Theorem 2.9 we have $\tau_{b} \tau_{a \circ x} b=$ $\tau_{b} \tau_{a} \tau_{x} b=\tau_{b} \tau_{a} b=a^{\prime}$. Therefore $\left(a \circ x \mid a^{\prime}\right)=\left(a \circ x \mid \tau_{b} \tau_{a \circ x} b\right)=2^{-5}$. Since $a$ and $b$ are fixed by $\tau_{x}, z=a \circ a^{\prime}$ is also fixed by $\tau_{x}$. Clearly $a$ and $a^{\prime}$ are fixed by $\left\langle\tau_{a}, \tau_{a^{\prime}}\right\rangle$ and hence $a, a^{\prime}, z \in V_{\mathbb{R}}^{G}$. Similarly, $x$ is fixed by $\left\langle\tau_{a}, \tau_{b}\right\rangle$ so that $x$ is fixed by $\tau_{a}$ and $\tau_{a^{\prime}}=\tau_{\tau_{b} \tau_{a} b}=\tau_{b} \tau_{a} \tau_{b} \tau_{a} \tau_{b}$. Therefore, all of $a, a^{\prime}, z$ and $x$ belong to $V_{\mathbb{R}}^{G}$ and of $\sigma$-type on it. We have $\left(a^{\prime} \mid x\right)=\left(\tau_{b} \tau_{a} b \mid x\right)=\left(b \mid \tau_{a} \tau_{b} x\right)=(b \mid x)=2^{-5}$ and $(z \mid x)=\left(a \circ a^{\prime} \mid x\right)=$ $\left(\sigma_{a^{\prime}} a \mid x\right)=\left(a \mid \sigma_{a^{\prime}} x\right)=\left(a \mid \sigma_{x} a^{\prime}\right)=\left(\sigma_{x} a \mid a^{\prime}\right)=\left(a \circ x \mid a^{\prime}\right)=2^{-5}$. Therefore, we have $\left(a \mid a^{\prime}\right)=(a \mid z)=(a \mid x)=\left(a^{\prime} \mid z\right)=\left(a^{\prime} \mid x\right)=(z \mid x)=2^{-5}$. Namely, $a, a^{\prime}, x$ and $z$ form a 2A-tetrahedron in $V_{\mathbb{R}}^{G}$ which contradicts Lemma 2.10. Thus $\tau_{x}=\tau_{z}$.

Lemma 3.4. Let $a, b, x, y$ be Ising vectors of $V$ such that $\langle a, x\rangle \cong\langle b, x\rangle \cong\langle a, y\rangle \cong\langle b, y\rangle \cong$ $U_{2 \mathrm{~A}}$ and $\langle x, y\rangle \cong U_{6 \mathrm{~A}}$. Then $\tau_{a}=\tau_{b}$.

Proof: Let $z$ be the central Ising vector of $\langle x, y\rangle$. Applying Lemma 3.3 to $x, y$, a, we obtain $\tau_{a}=\tau_{z}$, and applying Lemma 3.3 to $x, y, b$, we obtain $\tau_{b}=\tau_{z}$. Thus $\tau_{a}=\tau_{b}$. 
Let $E_{V}$ be the set of Ising vectors of $V_{\mathbb{R}}$. Suppose there is a pair $a, b \in E_{V}$ such that $\langle a, b\rangle \cong U_{3 \mathrm{~A}}$. Then we set

$$
I_{a, b}:=\left\{x \in E_{V} \mid\langle a, x\rangle \cong\langle b, x\rangle \cong U_{2 \mathrm{~A}}\right\}
$$

We will show that a 3-transposition group arises from $I_{a, b}$.

Theorem 3.5. Let $a, b \in V_{\mathbb{R}}$ be Ising vectors such that $\langle a, b\rangle \cong U_{3 \mathrm{~A}}$. Define $I_{a, b}$ as in (3.1). Then for any $x, y \in I_{a, b},\langle x, y\rangle \cong U_{1 \mathrm{~A}}, U_{2 \mathrm{~A}}$ or $U_{3 \mathrm{~A}}$.

Proof: Let $x, y \in I_{a, b}$. We eliminate the possibility of the type of $\langle x, y\rangle$ other than 1A, 2A, and 3A. By our choice, $\tau_{x}$ and $\tau_{y}$ commute with $\tau_{a}$ and $\tau_{b}$.

(i) Case 6A: Since $\tau_{a} \tau_{b}$ is non-trivial on $\langle a, b\rangle \cong U_{3 \mathrm{~A}}$, it is also non-trivial on $V$. If $\langle x, y\rangle \cong U_{6 \mathrm{~A}}$, then $\tau_{a}=\tau_{b}$ by Lemma 3.4 and we have a contradiction.

(ii) Case 5A: Suppose $\langle x, y\rangle \cong U_{5 \mathrm{~A}}$. Then $\tau_{x} \tau_{y}$ has an order 5. Since $\tau_{a}$ is non-trivial, $\tau_{a} \tau_{x} \tau_{y}=\tau_{a \circ x} \tau_{y}$ has order 10 and we have a contradiction by the 6 -transposition property in Theorem 2.7.

(iii) Cases $4 \mathrm{~A}$ and $4 \mathrm{~B}$ : Suppose $\langle x, y\rangle \cong U_{4 \mathrm{~A}}$ or $U_{4 \mathrm{~B}}$. Then $\tau_{x} \tau_{y}$ has order 2 or 4 by Theorem 2.7. By (5) of Theorem 2.9, we have

$$
\tau_{a \circ x} \tau_{b \circ y}=\tau_{a} \tau_{x} \tau_{b} \tau_{y}=\tau_{a} \tau_{b} \tau_{x} \tau_{y}
$$

If $\tau_{x} \tau_{y}$ has order 4 , then $\tau_{a \circ x} \tau_{b \circ y}$ has order 12 , which contradicts the 6 -transposition property in Theorem 2.7. If $\tau_{x} \tau_{y}$ has order 2 , then $\tau_{a \circ x} \tau_{b \circ y}$ has order 6 and the subVOA generated by $a \circ x$ and $b \circ y$ is isomorphic to the $6 \mathrm{~A}$-algebra. Since $\tau_{a \circ x} \tau_{b \circ y}$ has order 3 on $\langle a \circ x, b \circ y\rangle,\left(\tau_{a \circ x} \tau_{b \circ y}\right)^{3}=\tau_{x} \tau_{y}$ acts trivially on $\langle a \circ x, b \circ y\rangle$. Thus $\tau_{x} \tau_{y}$ fixes both $a \circ x$ and $b \circ y$. Since $\tau_{y}$ fixes $b \circ y$, so does $\tau_{x}$ and hence $\tau_{b \circ y}$ fixes $x$. Then $\tau_{y}$ fixes $x$ since $\tau_{b \circ y}=\tau_{y} \tau_{b}$ and $\tau_{b}$ fixes $x$. This contradicts (6) of Theorem 2.7. Hence, $\langle x, y\rangle$ cannot be the $4 \mathrm{~A}$ nor the $4 \mathrm{~B}$-algebra.

(iv) Case 3C: That $\langle x, y\rangle \cong U_{3 \mathrm{C}}$ is impossible by Lemma 3.2 since $\langle x, y\rangle \subset\langle a, x, y\rangle \cong U_{6 \mathrm{~A}}$.

(v) Case 2B: Suppose $\langle x, y\rangle \cong U_{2 \mathrm{~B}}$. Then $x_{(1)} y=0$ and $(x \mid y)=0$. It follows from $a \circ x=a+x-4 a_{(1)} x$ that

$$
(a \circ x \mid y)=(a \mid y)+(x \mid y)-4\left(a_{(1)} x \mid y\right)=(a \mid y)-4\left(a \mid x_{(1)} y\right)=2^{-5}-4(a \mid 0)=2^{-5} .
$$

We consider $(a \circ x \mid b \circ y)=\left(a+x-4 a_{(1)} x \mid b \circ y\right)=(a \mid b \circ y)+(x \mid b \circ y)-4\left(x \mid a_{(1)}(b \circ y)\right)$. By (7) of Theorem 2.13, we have

$$
a_{(1)}(b \circ y)=\frac{45}{2^{10}} u_{a, b}+\frac{1}{2^{5}}\left(y+a+b \circ y-a \circ y-b-\tau_{a} b-\left(\tau_{a} b\right) \circ y\right),
$$


where $u_{a, b}$ is the characteristic $c=4 / 5$ Virasoro vector in $\langle a, b\rangle$. Then we have

$$
\left(x \mid a_{(1)}(b \circ y)\right)=-2 \cdot 2^{-10}
$$

since $\left(u_{a, b} \mid x\right)=(y \mid x)=0$ and $(a \mid x)=(b \circ y \mid x)=(a \circ y \mid x)=(b \mid x)=\left(\tau_{a} b \mid x\right)=$ $\left(\left(\tau_{a} b\right) \circ y \mid x\right)=2^{-5}$. Thus,

$(a \circ x \mid b \circ y)=(a \mid b \circ y)+(x \mid b \circ y)-4\left(x \mid a_{(1)}(b \circ y)\right)=5 \cdot 2^{-10}+2^{-5}+8 \cdot 2^{-10}=45 \cdot 2^{-10}$,

which is impossible by Theorem 2.6.

Therefore, $\langle x, y\rangle$ is isomorphic to one of $U_{1 \mathrm{~A}}, U_{2 \mathrm{~A}}$ or $U_{3 \mathrm{~A}}$.

As a corollary, we have

Corollary 3.6. For $x, y \in I_{a, b}$, one has $\left|\tau_{x} \tau_{y}\right| \leq 3$. Therefore, $G=\left\langle\tau_{x} \mid x \in I_{a, b}\right\rangle$ forms a 3-transposition group in the stabilizer $\left\{g \in \operatorname{Aut}\left(V_{\mathbb{R}}\right) \mid g a=a, g b=b\right\}$ of $\langle a, b\rangle$.

\section{Inductive structures}

Let $V$ be an OZ-type VOA with a compact real form $V_{\mathbb{R}}$, and let $E_{V}$ be the set of Ising vectors in $V_{\mathbb{R}}$. Suppose we have a pair $a, b \in E_{V}$ such that $\langle a, b\rangle$ is isomorphic to the 3A-algebra, i.e., $(a \mid b)=13 \cdot 2^{-10}$, and consider the subset $I_{a, b}$ defined as in (3.1). Let $x$ and $y$ be distinct Ising vectors in $I_{a, b}$. In this section, we first study the subalgebra $\langle a, b, x, y\rangle$ and then introduce a series of subalgebras $X^{[i]}=\left\langle a, b, x^{1}, \ldots, x^{i}\right\rangle$ to study inductive structures of the 3 -transposition group obtained by Corollary 3.6. Note that $\langle x, y\rangle \cong U_{2 \mathrm{~A}}$ or $U_{3 \mathrm{~A}}$ by Theorem 3.5 .

Lemma 4.1. Let $a, x, y$ be Ising vectors such that $\langle a, x\rangle \cong\langle a, y\rangle \cong U_{2 \mathrm{~A}}$ and $a \notin\langle x, y\rangle$.

(1) If $\langle x, y\rangle$ is the 2A-algebra then $\left(a \mid x_{(1)} y\right)=2^{-6}$.

(2) If $\langle x, y\rangle$ is the $3 A$-algebra then $\left(a \mid x_{(1)} y\right)=5 \cdot 2^{-9}$.

Proof: (1): Suppose $\langle x, y\rangle$ is the 2A-algebra. Then $x_{(1)} y=\frac{1}{4}(x+y-x \circ y)$. By Lemma 2.10, one has $(a \mid x \circ y)=0$. Therefore

$$
\left(a \mid x_{(1)} y\right)=\frac{1}{4}(a \mid x+y-x \circ y)=\frac{1}{4} \cdot 2 \cdot 2^{-5}=2^{-6} .
$$

(2): Suppose $\langle x, y\rangle$ is the 3A-algebra. Then $\langle a, x, y\rangle$ is the $6 \mathrm{~A}$-algebra with the central Ising vector $a$ by Lemma 3.2. By (5) of Theorem 2.11, we have

$$
\left(a \mid x_{(1)} y\right)=-\frac{135}{2^{10}}\left(a \mid u_{x, y}\right)+\frac{1}{2^{4}}\left(a \mid 2 x+2 y+\tau_{x} y\right)=\frac{1}{2^{4}}(2+2+1) \cdot 2^{-5}=5 \cdot 2^{-9} .
$$

Thus we have the lemma. 
Proposition 4.2. Let $a, b, x, y$ be Ising vectors such that $\langle a, b\rangle \cong U_{3 \mathrm{~A}}$ and $\langle a, x\rangle \cong$ $\langle a, y\rangle \cong\langle b, x\rangle \cong\langle b, y\rangle \cong U_{2 \mathrm{~A}}$.

(1) If $\langle x, y\rangle$ is the 2A-algebra then $\langle a \circ x, b \circ y\rangle \cong U_{6 \mathrm{~A}}$, i.e., $(a \circ x \mid b \circ y)=5 \cdot 2^{-10}$.

(2) If $\langle x, y\rangle$ is the $3 A$-algebra then $\langle a \circ x, b \circ y\rangle \cong U_{3 \mathrm{~A}}$, i.e., $(a \circ x \mid b \circ y)=13 \cdot 2^{-10}$.

Proof: By definition, we have $a \circ x=a+x-4 a_{(1)} x$ and $b \circ y=b+y-4 b_{(1)} y$. So by Lemma 4.1, one has

$$
\begin{aligned}
(a \circ x \mid b \circ y)= & \left(a+x-4 a_{(1)} x \mid b+y-4 b_{(1)} y\right) \\
= & \underbrace{(a \mid b)}_{=13 \cdot 2^{-10}}+\underbrace{(a \mid y)}_{=2^{-5}}-4 \underbrace{\left(a_{(1)} b \mid y\right)}_{=5 \cdot 2^{-9}}+\underbrace{(x \mid b)}_{=2^{-5}}+(x \mid y)-4\left(x_{(1)} y \mid b\right) \\
& -4 \underbrace{\left(x \mid a_{(1)} b\right)}_{=5 \cdot 2^{-9}}-4\left(a \mid x_{(1)} y\right)+16\left(x \mid a_{(1)} b_{(1)} y\right) \\
= & (x \mid y)-4\left(a \mid x_{(1)} y\right)-4\left(b \mid x_{(1)} y\right)+16\left(x \mid a_{(1)} b_{(1)} y\right)-3 \cdot 2^{-10} .
\end{aligned}
$$

Notice that $\langle a, b, y\rangle$ is the $6 \mathrm{~A}$-algebra with the central Ising vector $y$ by Lemma 3.2. By Lemma 2.14 (see also Eq. (2.10)), we have

$$
\left(x \mid a_{(1)} b_{(1)} y\right)=2^{-7}\left(7(x \mid y)-7(x \mid a \circ y)-(x \mid b \circ y)+\left(x \mid \tau_{a} b \circ y\right)+19 \cdot 2^{-5}\right) .
$$

(1): If $\langle x, y\rangle$ is the 2A-algebra, then $(x \mid y)=2^{-5}$ and $\left(a \mid x_{(1)} y\right)=\left(b \mid x_{(1)} y\right)=2^{-6}$ by Lemma 4.1. Therefore

$$
(a \circ x \mid b \circ y)=16\left(x \mid a_{(1)} b_{(1)} y\right)-99 \cdot 2^{-10} .
$$

In this case, $(x \mid a \circ y)=0$ by Lemma 2.10 and $\tau_{a}$ acts trivially on $\langle x, y\rangle$. Thus $\tau_{a}(b \circ y)=$ $\tau_{a} b \circ \tau_{a} y=\tau_{a} b \circ y$ and $\left(x \mid \tau_{a} b \circ y\right)=\left(x \mid \tau_{a}(b \circ y)\right)=\left(\tau_{a} x \mid b \circ y\right)=(x \mid b \circ y)$. Then by (4.1), one has

$$
\left(x \mid a_{(1)} b_{(1)} y\right)=2^{-7}\left(7 \cdot 2^{-5}+19 \cdot 2^{-5}\right)=13 \cdot 2^{-11} .
$$

Therefore,

$$
(a \circ x \mid b \circ y)=16 \cdot 13 \cdot 2^{-11}-99 \cdot 2^{-10}=5 \cdot 2^{-10} .
$$

(2): If $\langle x, y\rangle$ is the $3 \mathrm{~A}$-algebra then $(x \mid y)=13 \cdot 2^{-10}$ and $\left(a \mid x_{(1)} y\right)=\left(b \mid x_{(1)} y\right)=5 \cdot 2^{-9}$ by Lemma 4.1. Therefore

$$
(a \circ x \mid b \circ y)=16\left(x \mid a_{(1)} b_{(1)} y\right)-35 \cdot 2^{-9} .
$$

In this case, $\langle x, a \circ y\rangle,\langle x, b \circ y\rangle$ and $\left\langle x, \tau_{a} b \circ y\right\rangle$ are all isomorphic to the $6 \mathrm{~A}$-algebra by Lemma 3.2. Thus $(x \mid a \circ y)=(x \mid b \circ y)=\left(x \mid \tau_{a} b \circ y\right)=5 \cdot 2^{-10}$. By (4.1), one has

$$
\left(x \mid a_{(1)} b_{(1)} y\right)=2^{-7}\left(7 \cdot 13 \cdot 2^{-10}-7 \cdot 5 \cdot 2^{-10}+19 \cdot 2^{-5}\right)=83 \cdot 2^{-14} .
$$


Therefore,

$$
(a \circ x \mid b \circ y)=16 \cdot 83 \cdot 2^{-14}-35 \cdot 2^{-9}=13 \cdot 2^{-10} .
$$

This completes the proof.

The following lemma is a slight modification of Lemma 3 of [Ma05] and is useful in determining the conformal vector of a subVOA.

Lemma 4.3. Let $V_{\mathbb{R}}$ be a compact VOA of OZ-type and let $A$ be a set of Virasoro vectors of $V_{\mathbb{R}}$ such that the linear span of $A$ forms a subalgebra of the Griess algebra. Then the subalgebra $\langle A\rangle$ generated by $A$ has a conformal vector and forms a subVOA of $V_{\mathbb{R}}$. The conformal vector $\eta$ of $\langle A\rangle$ belongs to the linear span of $A$ and uniquely determined by the condition $(\eta \mid a)=(a \mid a)$ for all $a \in A$.

Proof: First, we prove that $\langle A\rangle$ has the conformal vector inside $\mathbb{R} A$, the linear span of $A$. Let $\omega$ be the conformal vector of $V_{\mathbb{R}}$ and let $B$ be the orthogonal complement of $\mathbb{R} A$ in $\left(V_{\mathbb{R}}\right)_{2}$. Let $\omega=\eta+\xi$ be the orthogonal decomposition with respect to $\left(V_{\mathbb{R}}\right)_{2}=\mathbb{R} A \perp B$. For any $a \in A$, one has

$$
\left(a_{(1)} B \mid \mathbb{R} A\right)=\left(B \mid a_{(1)} \mathbb{R} A\right) \subset(B \mid \mathbb{R} A)=0 .
$$

Therefore $(\mathbb{R} A)_{(1)} B \subset B$. Then

$$
2 \eta=\omega_{(1)} \eta=(\eta+\xi)_{(1)} \eta=\eta_{(1)} \eta+\eta_{(1)} \xi
$$

and we obtain $\eta_{(1)} \xi=2 \eta-\eta_{(1)} \eta \in B \cap \mathbb{R} A=0$. Thus $\eta_{(1)} \eta=2 \eta$ and $\eta_{(1)} \xi=0$. This implies $\xi_{(1)} \xi=(\omega-\eta)_{(1)} \xi=2 \xi$ and hence $\eta$ and $\xi$ are Virasoro vectors of $V_{\mathbb{R}}$ by Lemma 5.1 of [Mi96]. Since a Virasoro vector $\eta$ satisfies $\eta_{(0)} \eta=\eta_{(-2)} \mathbb{1}$, we have

$$
\xi_{(0)} \eta=(\omega-\eta)_{(0)} \eta=\omega_{(0)} \eta-\eta_{(0)} \eta=\eta_{(-2)} \mathbb{1}-\eta_{(-2)} \mathbb{1}=0
$$

and thus $\eta$ and $\xi$ are mutually orthogonal by Theorem 5.1 of [FZ92]. We will show that $\eta$ is the conformal vector of $\langle A\rangle$. For this, it suffices to show that $\eta_{(1)} a=2 a$ and $\eta_{(0)} a=a_{(-2)} \mathbb{1}$ for $a \in A$ since $\eta_{(0)}$ is a derivation on $V_{\mathbb{R}}$. Let $a \in A$. Then

$$
2 a=\omega_{(1)} a=(\eta+\xi)_{(1)} a=\eta_{(1)} a+\xi_{(1)} a
$$

and we have $\xi_{(1)} a=2 a-\eta_{(1)} a \in B \cap \mathbb{R} A=0$. Therefore $\eta_{(1)} a=2 a$ and $\xi_{(1)} a=0$. Next we will prove $\eta_{(0)} a=a_{(-2)} \mathbb{1}$. Since $\xi_{(2)} a \in\left(V_{\mathbb{R}}\right)_{1}=0$, we have

$$
\left(\xi_{(0)} a \mid \xi_{(0)} a\right)=\left(a \mid \xi_{(2)} \xi_{(0)} a\right)=\left(a \mid\left[\xi_{(2)}, \xi_{(0)}\right] a\right)=\left(a \mid 2 \xi_{(1)} a\right)=0
$$

and $\xi_{(0)} a=0$ by the positive definiteness of $V_{\mathbb{R}}$. Then $\eta_{(0)} a=(\omega-\xi)_{(0)} a=\omega_{(0)} a-\xi_{(0)} a=$ $\omega_{(0)} a=a_{(-2)} \mathbb{1}$. Therefore, $\eta$ is the conformal vector of $\langle A\rangle$. 
Finally, we will show that $\eta \in \mathbb{R} A$ is uniquely determined by the condition $(\eta \mid a)=$ $(a \mid a)$ for all $a \in A$. Since $\eta_{(1)} a=a_{(1)} a=2 a$, one has

$$
2(a \mid a)=\left(\eta_{(1)} a \mid a\right)=\left(\eta \mid a_{(1)} a\right)=2(\eta \mid a)
$$

and the conformal vector $\eta \in \mathbb{R} A$ satisfies the condition. Let $a_{1}, \ldots, a_{n} \in A$ be a linear basis of $\mathbb{R} A$ and write $\eta=c_{1} a_{1}+\cdots c_{n} a_{n}$ with $c_{1}, \ldots, c_{n} \in \mathbb{R}$. Then the Gram matrix $\left[\left(a_{i} \mid a_{j}\right)\right]_{1 \leq i, j \leq n}$ is non-singular and the condition $\left(\eta \mid a_{i}\right)=\left(a_{i} \mid a_{i}\right)$ uniquely determines the coefficients $c_{i} \in \mathbb{R}$. Therefore, the conformal vector of $\langle A\rangle$ is determined by the condition $\eta \in \mathbb{R} A$ and $(\eta \mid a)=(a \mid a)$ for all $a \in A$.

Remark 4.4. From the proof of Lemma 4.3, one sees that $\langle A\rangle \subset \operatorname{Com}_{V_{\mathbb{R}}}\langle\xi\rangle=\operatorname{Ker}_{V_{\mathbb{R}}} \xi_{(0)}$ (cf. Theorem 5.1 of [FZ92]). It is shown in Proposition 4 of [Ma05] that $\mathbb{R} A=\langle A\rangle \cap\left(V_{\mathbb{R}}\right)_{2}$ if $\mathbb{R} A=\left(V_{\mathbb{R}}\right)_{2} \cap \operatorname{Ker}_{V} \xi_{(1)}$.

\subsection{The case $\langle x, y\rangle \cong U_{2 \mathrm{~A}}$}

In this subsection, we determine the Griess algebra of $\langle a, b, x, y\rangle$ when $\langle x, y\rangle$ is the $2 \mathrm{~A}$ algebra.

Theorem 4.5. Let $a, b, x, y$ be Ising vectors such that $\langle a, b\rangle$ is isomorphic to the $3 A$ algebra, and $\langle x, y\rangle,\langle a, x\rangle,\langle a, y\rangle,\langle b, x\rangle$ and $\langle b, y\rangle$ are isomorphic to the 2A-algebra, i.e., $(x \mid y)=(a \mid x)=(a \mid y)=(b \mid x)=(b \mid y)=2^{-5}$. Then the Griess subalgebra of $\langle a, b, x, y\rangle$ generated by $a, b, x, y$ is 13-dimensional with a basis

$$
A=\left\{u=u_{a, b}, a, b, c=\tau_{a} b, a \circ x, b \circ x, c \circ x, a \circ y, b \circ y, c \circ y, x, y, z=x \circ y\right\} .
$$

The conformal vector of $\langle a, b, x, y\rangle$ is

$$
\eta=\frac{1}{6} u+\frac{16}{27}(a+b+c+a \circ x+b \circ x+c \circ x+a \circ y+b \circ y+c \circ y)+\frac{4}{9}(x+y+z)
$$

and has 52/15. The VOA $\langle a, b, x, y\rangle$ has a full subVOA isomorphic to

$$
L\left(c_{3}, 0\right) \otimes L\left(c_{4}, 0\right) \otimes L\left(c_{5}, 0\right) \otimes L\left(c_{6}, 0\right)=L(4 / 5,0) \otimes L(6 / 7,0) \otimes L(25 / 28,0) \otimes L(11 / 12,0) .
$$

Proof: Set $c=\tau_{a} b=\tau_{b} a$ and $z=x \circ y$. We have seen in Lemma 3.2 that both $\langle a, b \circ x\rangle$ and $\langle a, b \circ y\rangle$ are isomorphic to the 6A-algebra and

$$
\begin{aligned}
& \langle a, b \circ x\rangle_{2}=\operatorname{Span}\left\{u_{a, b}, x, a, b, c, a \circ x, b \circ x, c \circ x\right\}, \\
& \langle a, b \circ y\rangle_{2}=\operatorname{Span}\left\{u_{a, b}, y, a, b, c, a \circ y, b \circ y, c \circ y\right\} .
\end{aligned}
$$

We have $u_{a, b}=u_{a \circ x, b \circ x}$ in $\langle a, b \circ x\rangle$ and $u_{a, b}=u_{a \circ y, b \circ y}$ in $\langle a, b \circ y\rangle$. Therefore

$$
u=u_{a, b}=u_{a \circ x, b \circ x}=u_{a \circ y, b o y} .
$$


It follows from Lemma 2.10 that $(a \mid x \circ y)=(x \mid a \circ y)=(y \mid a \circ x)=0$ so that $\sigma_{a}(x \circ y)=$ $x \circ y$ and

$$
(a \circ x) \circ(a \circ y)=\left(\sigma_{a} x\right) \circ\left(\sigma_{a} y\right)=\sigma_{a}(x \circ y)=x \circ y .
$$

Therefore, the 3 -set $\{a \circ x, a \circ y, x \circ y\}$ forms a 2 A-triple. Similarly, the 3 -sets $\{b \circ x, b \circ y, x \circ y\}$ and $\{c \circ x, c \circ y, x \circ y\}$ also form 2A-triples. By (1) of Proposition 4.2, the Griess algebra of the subalgebra $\langle a \circ x, b \circ y\rangle$ is isomorphic to the $6 \mathrm{~A}$-algebra with a basis

$$
\begin{aligned}
& a \circ x, b \circ y, \tau_{a \circ x}(b \circ y)=c \circ y, \tau_{b \circ y}(a \circ x)=c \circ x, \tau_{c \circ x}(b \circ y)=a \circ y, \\
& \tau_{c \circ y}(a \circ x)=b \circ x,(a \circ x) \circ(a \circ y)=x \circ y=z, u_{a \circ x, b \circ x}=u_{a \circ y, b \circ y}=u_{a, b} .
\end{aligned}
$$

Therefore, the linear span of $A$ forms a subalgebra in the Griess algebra. The determinant of the Gram matrix of $A$ is $3^{25} \cdot 11^{5} / 2^{81} \cdot 5$ so that this matrix is non-singular. Thus $A$ is a basis of the Griess subalgebra generated by $a, b, x$ and $y$.

One can directly verify that the vector $\eta$ satisfies $(\eta \mid t)=(t \mid t)$ for all $t \in A$. Therefore, $\eta$ is the conformal vector of $\langle a, b, x, y\rangle$ by Lemma 4.3. The central charge of $\eta$ is $2(\eta \mid \eta)=52 / 15$. We know that the subalgebra $\langle a, b, x\rangle$ is isomorphic to the 6 A-algebra and has a Virasoro frame $L(4 / 5,0) \otimes L(6 / 7,0) \otimes L(25 / 28,0)$ (see (8) of Theorem 2.13). By a direct computation, one finds that the commutant subalgebra of $\langle a, b, x\rangle$ in $\langle a, b, x, y\rangle$ is generated by the following $c=11 / 12$ Virasoro vector:

$$
f=-\frac{5}{24} u-\frac{2}{27}(a+b+c+a \circ x+b \circ x+c \circ x)+\frac{16}{27}(a \circ y+b \circ y+c \circ y)-\frac{1}{18} x+\frac{4}{9}(y+z) .
$$

Therefore, $\langle a, b, x, y\rangle$ has a unitary Virasoro frame isomorphic to

$$
L\left(c_{3}, 0\right) \otimes L\left(c_{4}, 0\right) \otimes L\left(c_{5}, 0\right) \otimes L\left(c_{6}, 0\right)=L(4 / 5,0) \otimes L(6 / 7,0) \otimes L(25 / 28,0) \otimes L(11 / 12,0) .
$$

This completes the proof.

\subsection{The case $\langle x, y\rangle \cong U_{3 \mathrm{~A}}$}

Next we will determine the Griess algebra for $\langle a, b, x, y\rangle$ when $\langle x, y\rangle \cong U_{3 \mathrm{~A}}$. The result will be useful for analyzing the structures of the 3-transposition groups mentioned in Corollary 3.6. However, we are mainly interested in the inductive structures, i.e., the case when $\tau_{x}$ and $\tau_{y}$ commute. Thus, the results in this subsection will not be used in the later section. We include this subsection for completeness and for future references.

Suppose $\langle a, b\rangle$ and $\langle x, y\rangle$ are isomorphic to the 3A-algebra and $\langle a, x\rangle,\langle a, y\rangle,\langle b, x\rangle$ and $\langle b, y\rangle$ are isomorphic the 2A-algebra, i.e., $(a \mid x)=(a \mid y)=(b \mid x)=(b \mid y)=2^{-5}$. Let $c=\tau_{a} b=\tau_{b} a$ and $z=\tau_{x} y=\tau_{y} x$. We also recall from (2.8) that $u_{a, b}$ and $u_{x, y}$ denote the characteristic $c=4 / 5$ Virasoro vectors in the 3A-algebras $\langle a, b\rangle$ and $\langle x, y\rangle$, respectively. Since all of $\langle a, b, x\rangle,\langle a, b, y\rangle,\langle a, b, z\rangle,\langle a, x, y\rangle,\langle b, x, y\rangle$ and $\langle c, x, y\rangle$ are isomorphic to the 
6A-algebra by Lemma 3.2 and $U_{6 \mathrm{~A}}$ has the unique characteristic $c=4 / 5$ Virasoro vector, we have

$$
u_{a, b}=u_{a \circ x, b \circ x}=u_{a \circ y, b \circ y}=u_{a \circ z, b \circ z}, \quad u_{x, y}=u_{a \circ x, a \circ y}=u_{b \circ x, b \circ y}=u_{c \circ x, c \circ y} .
$$

Consider the subalgebra generated by $a \circ x, a \circ y$ and $b \circ x$. Since $\tau_{a \circ x}=\tau_{a} \tau_{x}, \tau_{a \circ y}=\tau_{a} \tau_{y}$ and $\tau_{b \circ x}=\tau_{b} \tau_{x}$ by (5) of Theorem 2.11, we have $\tau_{a \circ x} \tau_{a \circ y}=\tau_{x} \tau_{y}$ and $\tau_{a \circ x} \tau_{b \circ x}=\tau_{a} \tau_{b}$ and obtain the following conjugacy.

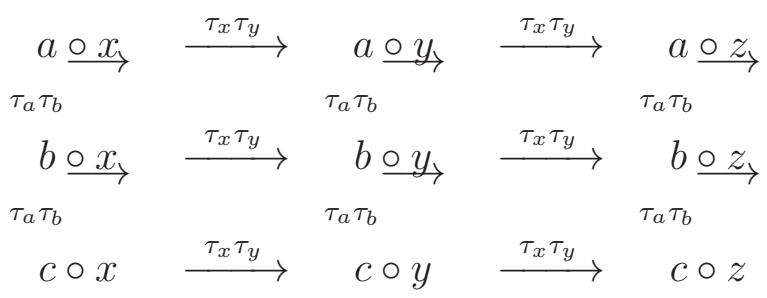

Set $H=\left\langle\tau_{a \circ x}, \tau_{a \circ y}, \tau_{b \circ x}\right\rangle$. Then $H \cong 3^{2}: 2$ acts transitively on the the set of 9 Ising vectors in (4.4). By (2) of Proposition 4.2, we see that $(a \circ x \mid b \circ y)=13 \cdot 2^{-10}$ and hence $\langle a \circ x, b \circ y\rangle$ is also a 3A-algebra. We have $\tau_{a \circ x}(b \circ y)=\tau_{a} b \circ \tau_{x} y=c \circ z$ and $\{a \circ y, b \circ x, c \circ z\}$ forms the 3A-triple of $\langle a \circ y, b \circ x\rangle$. Similarly, by using the conjugacy relations we can verify that each pair of Ising vectors in (4.4) generate a $3 \mathrm{~A}$-algebra and we obtain the following four $H$-orbits of $3 \mathrm{~A}$-triples:

$$
\begin{aligned}
& \mathcal{L}_{1}=\{\{a \circ x, b \circ x, c \circ x\},\{a \circ y, b \circ y, c \circ y\},\{a \circ z, b \circ z, c \circ z\}\}, \\
& \mathcal{L}_{2}=\{\{a \circ x, a \circ y, a \circ z\},\{b \circ x, b \circ y, b \circ z\},\{c \circ x, c \circ y, c \circ z\}\}, \\
& \mathcal{L}_{3}=\{\{a \circ x, b \circ y, c \circ z\},\{a \circ y, b \circ z, c \circ x\},\{a \circ z, b \circ x, c \circ y\}\}, \\
& \mathcal{L}_{4}=\{\{a \circ x, b \circ z, c \circ y\},\{a \circ y, b \circ x, c \circ z\},\{a \circ z, b \circ y, c \circ x\}\} .
\end{aligned}
$$

The configuration of 9 points given by Ising vectors in (4.4) together with the lines given by 3 -sets forming $3 \mathrm{~A}$-triples is isomorphic to the affine plane of order 3 and each $H$-orbit $\mathcal{L}_{i}$ in (4.5) is the set of parallel lines. By (4.3), all 3A-triples in $\mathcal{L}_{1}$ define the same characteristic $c=4 / 5$ Virasoro vector. The subVOA generated by such 9 Ising vectors is determined in $[\mathrm{LSu}]$.

Lemma 4.6 (Lemma 4.22 and Proposition 4.24 of $[\mathrm{LSu}]$ ). For $1 \leq i \leq 4$, all $3 A$-triples in $\mathcal{L}_{i}$ define the same characteristic $c=4 / 5$ Virasoro vector $u^{i}$. Moreover, the $c=4 / 5$ Virasoro vectors $u^{i}, 1 \leq i \leq 4$, are mutually orthogonal.

As a consequence, we have

$$
u_{a \circ x, b \circ y}=u_{a \circ y, b \circ z}=u_{a \circ z, b \circ x}, \quad \text { and } \quad u_{a \circ x, b \circ z}=u_{a \circ y, b \circ x}=u_{a \circ z, b \circ y} .
$$

It follows from the relations above that the linear span of the Ising vectors in (4.4) together with the $c=4 / 5$ Virasoro vectors $u^{1}=u_{a, b}, u^{2}=u_{x, y}, u^{3}=u_{a \circ x, b \circ y}$ and $u^{4}=u_{a \circ x, b \circ z}$ 
forms a subalgebra in the Griess algebra. The products and inner products of this Griess subalgebra is described by using the 3A-algebras along with the incidence structure of the affine plane of order 3. More precisely, the subalgebra generated by Ising vectors in (4.4) is described as follows.

Theorem 4.7 (Lemma 3.8 of [HLY12b] and Theorem 4.25 of [LSu]). The subVOA generated by 9 Ising vectors in (4.4) is isomorphic the ternary code VOA $M_{\mathcal{C}}$ constructed in [KMY00]. It is actually generated by 3 Ising vectors $a \circ x, a \circ y$ and $b \circ x$ and its Griess algebra is 12-dimensional spanned by 9 Ising vectors in (4.4) and 4 mutually orthogonal $c=4 / 5$ Virasoro vectors $u_{a, b}, u_{x, y}, u_{a \circ x, b \circ y}$ and $u_{a \circ x, b \circ z}$ with one linear relation

$$
\begin{aligned}
& u_{a, b}+u_{x, y}+u_{a \circ x, b \circ y}+u_{a \circ x, b \circ z} \\
& =\frac{32}{45}(a \circ x+b \circ x++c \circ x+a \circ y+b \circ y+c \circ y+a \circ z+b \circ z+c \circ z) .
\end{aligned}
$$

Moreover, $\omega=u_{a, b}+u_{x, y}+u_{a \circ x, b \circ y}+u_{a \circ x, b \circ z}$ gives the conformal vector of the subalgebra.

Set

$$
\begin{aligned}
A=\left\{u_{a, b}, u_{x, y},\right. & u_{a \circ x, b \circ y}, u_{a \circ x, b \circ z}, a, b, c=\tau_{a} b, x, y, z=\tau_{x} y, \\
& \circ \circ x, a \circ y, a \circ z, b \circ x, b \circ y, c \circ z, c \circ x, c \circ y, c \circ z\} .
\end{aligned}
$$

In the rest of this subsection, we will prove that the linear span of $A$ forms a subalgebra in the Griess algebra. By Lemma 3.2, the following subalgebras are isomorphic to the Griess algebra of the 6A-algebra:

$$
\begin{aligned}
& \langle a, b, x\rangle_{2}=\operatorname{Span}\left\{u_{a, b}, x, a, b, c, a \circ x, b \circ x, c \circ x\right\}, \\
& \langle a, b, y\rangle_{2}=\operatorname{Span}\left\{u_{a, b}, y, a, b, c, a \circ y, b \circ y, c \circ y\right\}, \\
& \langle a, b, z\rangle_{2}=\operatorname{Span}\left\{u_{a, b}, z, a, b, c, a \circ z, b \circ z, c \circ z\right\}, \\
& \langle a, x, y\rangle_{2}=\operatorname{Span}\left\{u_{x, y}, a, x, y, z, a \circ x, a \circ y, a \circ z\right\}, \\
& \langle b, x, y\rangle_{2}=\operatorname{Span}\left\{u_{x, y}, b, x, y, z, b \circ x, b \circ y, b \circ z\right\}, \\
& \langle c, x, y\rangle_{2}=\operatorname{Span}\left\{u_{x, y}, c, x, y, z, c \circ x, c \circ y, c \circ z\right\} .
\end{aligned}
$$

Therefore, it is enough to show that the products of $a, b, c, x, y, z$ and $u_{a \circ x, b \circ y}, u_{a \circ x, b \circ z}$ lie in the linear span of $A$ and determine the inner products among them. Set $G=\left\langle\tau_{a}, \tau_{b}, \tau_{x}, \tau_{y}\right\rangle$. Then the sets of $3 \mathrm{~A}$-triples $\mathcal{L}_{1}$ and $\mathcal{L}_{2}$ in $(4.5)$ are $G$-stable, whereas $\mathcal{L}_{3}$ and $\mathcal{L}_{4}$ are $H$ stable but not $G$-stable. We have the following conjugacy.

Lemma 4.8. For $e \in\{a, b, c, x, y, z\}$, we have $\tau_{e} u_{a \circ x, b \circ y}=u_{a \circ x, b \circ z}$.

Proof: Let $e \in\{a, b, c, x, y, z\}$. Then we have $\tau_{e} \mathcal{L}_{3}=\mathcal{L}_{4}$ and the conjugacy $\tau_{e} u_{a \circ x, b \circ y}=$ $u_{a \circ x, b \circ z}$ of the characteristic $c=4 / 5$ Virasoro vectors follows from that of the corresponding 3A-triples. 
Lemma 4.9. Let $e \in\{x, y, z, a, b, c\}$. Then

$$
\left(e \mid u_{a \circ x, b \circ y}\right)=\left(e \mid u_{a \circ x, b \circ z}\right)=\frac{1}{80} .
$$

Proof: Recall that

$$
u_{a \circ x, b \circ y}=\frac{2^{6}}{135}\left(2 a \circ x+2 b \circ y+c \circ z-16(a \circ x)_{(1)}(b \circ y)\right) .
$$

First we have

$$
\begin{aligned}
\left(a \mid(a \circ x)_{(1)}(b \circ y)\right) & =\left(a_{(1)}(a \circ x) \mid b \circ y\right)=\frac{1}{4}(a+a \circ x-x \mid b \circ y) \\
& =\frac{1}{4}\left(\frac{5}{2^{10}}+\frac{13}{2^{10}}-\frac{5}{2^{10}}\right)=\frac{13}{2^{12}} .
\end{aligned}
$$

Hence,

$$
\left(a \mid u_{a \circ x, b \circ y}\right)=\frac{2^{6}}{135}\left(2 \cdot \frac{1}{2^{5}}+2 \cdot \frac{5}{2^{10}}+\frac{5}{2^{10}}-16 \cdot \frac{13}{2^{12}}\right)=\frac{1}{80} .
$$

The other cases can be proved similarly.

Let us compute $e_{(1)} u$ for $e \in\{a, b, c, x, y, z\}$ and $u \in\left\{u_{a \circ x, b \circ y}, u_{a \circ x, b \circ z}\right\}$. Recall that $a \circ x=2^{-2}\left(a+x-a_{(1)} x\right)$ in (2.7). For simplicity, we regard $a \circ x$ as a bilinear product and define $a \circ(x+y+z)=a \circ x+a \circ y+a \circ z$.

Lemma 4.10. Let $u=u_{a \circ x, b \circ y}$ or $u_{a \circ x, b \circ z}$. Then we have

$$
\begin{aligned}
\sigma_{e}\left(u+\tau_{e} u\right)=- & \frac{1}{2} u_{a, b}-u_{x, y}+\frac{4}{15} e-\frac{8}{45} e \circ(x+y+z) \\
& -\frac{16}{45}(a+b+c)+\frac{8}{15}(x+y+z)+\frac{32}{45}(a+b+c) \circ(x+y+z)
\end{aligned}
$$

if $e \in\{a, b, c\}$, and

$$
\begin{aligned}
\sigma_{e}\left(u+\tau_{e} u\right)=- & u_{a, b}-\frac{1}{2} u_{x, y}+\frac{4}{15} e-\frac{8}{15}(a+b+c) \circ e \\
& +\frac{8}{15}(a+b+c)-\frac{16}{45}(x+y+z)+\frac{32}{45}(a+b+c) \circ(x+y+z)
\end{aligned}
$$

if $e \in\{x, y, z\}$.

Proof: We only compute the case $e=x$. The case $e=a$ is similar. By (2.8), we have

$$
\begin{aligned}
& u_{a \circ x, b \circ y}=\frac{2^{6}}{135}\left(2 a \circ x+2 b \circ y+c \circ z-16(a \circ x)_{(1)}(b \circ y)\right), \\
& u_{a \circ x, b \circ z}=\frac{2^{6}}{135}\left(2 a \circ x+2 b \circ z+c \circ y-16(a \circ x)_{(1)}(b \circ z)\right) .
\end{aligned}
$$


Since $\sigma_{x}(a \circ x)=a$ and $u+\tau_{x} u=u_{a \circ x, b \circ y}+u_{a \circ x, b \circ z}$, we have

$$
\begin{aligned}
& \sigma_{x}\left(u+\tau_{x} u\right)=\sigma_{x}\left(u_{a \circ x, b \circ y}+u_{a \circ x, b \circ z}\right) \\
& =\frac{2^{6}}{135} \sigma_{x}\left(4 a \circ x+2 b \circ y+2 b \circ z+c \circ y+c \circ z-16(a \circ x)_{(1)}(b \circ y+b \circ z)\right) \\
& =\frac{2^{6}}{135}\left(4 a+2 \sigma_{x}(b \circ y+b \circ z)+\sigma_{x}(c \circ y+c \circ z)-16 a_{(1)} \sigma_{x}(b \circ y+b \circ z)\right) .
\end{aligned}
$$

By Lemma 2.14 (cf. Eq. (2.10)), we have

$$
\begin{aligned}
& \sigma_{x}(b \circ y+b \circ z)=-\frac{45}{2^{7}} u_{x, y}-\frac{1}{2^{2}} b+\frac{1}{2^{4}} x+\frac{1}{2^{2}} b \circ x+\frac{1}{2^{2}}(y+z)+b \circ y+b \circ z, \\
& \sigma_{x}(c \circ y+c \circ z)=-\frac{45}{2^{7}} u_{x, y}-\frac{1}{2^{2}} c+\frac{1}{2^{4}} x+\frac{1}{2^{2}} c \circ x+\frac{1}{2^{2}}(y+z)+c \circ y+c \circ z .
\end{aligned}
$$

By using the Griess algebras of $\langle a, b \circ y\rangle$ and $\langle a, b \circ z\rangle$ (cf. (7) of Theorem 2.13), we have

$$
\begin{aligned}
a_{(1)} & \sigma_{x}(b \circ y+b \circ z) \\
= & a_{(1)}\left(-\frac{45}{2^{7}} u_{x, y}-\frac{1}{2^{2}} b+\frac{1}{2^{4}} x+\frac{1}{2^{2}} b \circ x+\frac{1}{2^{2}}(y+z)+b \circ y+b \circ z\right) \\
= & -\frac{45}{2^{7}} a_{(1)} u_{x, y}-\frac{1}{2^{2}}\left(-\frac{135}{2^{10}} u_{a, b}+\frac{1}{2^{4}}(2 a+2 b+c)\right)+\frac{1}{2^{4}} \cdot \frac{1}{4}(a+x-a \circ x) \\
& +\frac{1}{2^{2}}\left(\frac{45}{2^{10}} u_{a, b}+\frac{1}{2^{5}}(x+a+b \circ x-b-c-a \circ x-c \circ x)\right) \\
& +\frac{1}{2^{2}}\left(\frac{1}{4}(a+y-a \circ y)+\frac{1}{4}(a+z-a \circ z)\right) \\
& +\frac{45}{2^{10}} u_{a, b}+\frac{1}{2^{5}}(y+a+b \circ y-b-c-a \circ y-c \circ y) \\
& +\frac{45}{2^{10}} u_{a, b}+\frac{1}{2^{5}}(z+a+b \circ z-b-c-a \circ z-c \circ z) .
\end{aligned}
$$

Note that $a_{(1)} u_{x, y}=0$ in the 6A-algebra $\langle a, x, y\rangle$ and thus by plugging (4.10) and (4.11) into (4.9), we have

$$
\begin{aligned}
\sigma_{x}\left(u+\tau_{x} u\right)= & \frac{8}{15}(a+b+c)-\frac{4}{45} x-\frac{16}{45}(y+z)+\frac{8}{45}(a \circ x+b \circ x+c \circ x) \\
& +\frac{32}{45}(a \circ y+b \circ y+c \circ y+a \circ z+b \circ z+c \circ z)-u_{a, b}-\frac{1}{2} u_{x, y}
\end{aligned}
$$

as desired.

Lemma 4.11. Let $u=u_{a \circ x, b \circ y}$ or $u_{a \circ x, b \circ z}$. Then we have

$$
\begin{aligned}
e_{(1)} u= & \frac{1}{16} u_{a, b}+\frac{1}{8} u_{x, y}+\frac{5}{32} u+\frac{3}{32} \tau_{e} u+\frac{1}{15} e+\frac{1}{15} e \circ(x+y+z) \\
& +\frac{2}{45}(a+b+c)-\frac{1}{15}(x+y+z)-\frac{4}{45}(a+b+c) \circ(x+y+z)
\end{aligned}
$$


if $e \in\{a, b, c\}$, and

$$
\begin{aligned}
e_{(1)} u= & \frac{1}{8} u_{a, b}+\frac{1}{16} u_{x, y}+\frac{5}{32} u+\frac{3}{32} \tau_{e} u+\frac{1}{15} e+\frac{1}{15}(a+b+c) \circ e \\
& -\frac{1}{15}(a+b+c)+\frac{2}{45}(x+y+z)-\frac{4}{45}(a+b+c) \circ(x+y+z)
\end{aligned}
$$

if $e \in\{x, y, z\}$.

Proof: Again we only compute the case $e=x$. By (2.5), we have

$$
x_{(1)} u=8(x \mid u) x+\frac{5}{32} u+\frac{3}{32} \tau_{x} u-\frac{1}{8} \sigma_{x}\left(u+\tau_{x} u\right) .
$$

Then the lemma immediately follows from Lemmas 4.9 and 4.10.

Recall the set $A$ of Virasoro vectors in (4.7). By Lemma 4.11 and $H$-invariance of $u_{a \circ x, b \circ y}$ and $u_{a \circ x, b \circ z}$, the products $e_{(1)} u$ for $e \in\{a, b, c, x, y, z\}$ and $u \in\left\{u_{a \circ x, b \circ y}, u_{a \circ x, b \circ z}\right\}$ are completely determined by linear combinations of the Virasoro vectors in $A$. Therefore, the linear span of $A$ forms a subalgebra of the Griess algebra of $\langle a, b, x, y\rangle$.

Next we determine the conformal vector of $\langle a, b, x, y\rangle$.

Proposition 4.12. Set

$$
\eta=\frac{17}{22}\left(u_{a, b}+u_{x, y}\right)+\frac{10}{11}\left(u_{a \circ x, b \circ y}+u_{a \circ x, b \circ z}\right)+\frac{16}{33}(a+b+c+x+y+z) .
$$

Then $(\eta \mid t)=(t \mid t)$ for all $t \in A$ and therefore $\eta$ is the conformal vector of $\langle a, b, x, y\rangle$ of central charge $228 / 55$.

Proof: Since $u_{a, b}, u_{x, y}, u_{a \circ x, b \circ y}, u_{a \circ x, b \circ z}$ are mutually orthogonal by Lemma 4.6, it is straightforward to verify $(\eta \mid t)=(t \mid t)$ for all $t \in A$ by using the 6A-algebras given in (4.8) and Lemma 4.9. By Lemma 4.3, $\eta$ is the conformal vector of $\langle a, b, x, y\rangle$. The central charge is given by

$$
\begin{aligned}
2(\eta \mid \eta) & =\frac{17}{22}\left(\eta \mid u_{a, b}+u_{x, y}\right)+\frac{10}{11}\left(\eta \mid u_{a \circ x, b \circ y}+u_{a \circ x, b \circ z}\right)+\frac{16}{33}(\eta \mid a+b+c+x+y+z) \\
& =2 \cdot\left(\frac{17}{22} \cdot \frac{2}{5} \cdot 2+\frac{10}{11} \cdot \frac{2}{5} \cdot 2+\frac{16}{33} \cdot \frac{1}{4} \cdot 6\right)=\frac{228}{55}
\end{aligned}
$$

as claimed.

Summarizing everything, the structure of the Griess algebra of $\langle a, b, x, y\rangle$ is described as follows.

Theorem 4.13. Let $a, b, x, y$ be Ising vectors of $V_{\mathbb{R}}$ such that $\langle a, b\rangle$ and $\langle x, y\rangle$ are isomorphic to the 3A-algebra and $\langle a, x\rangle,\langle a, y\rangle,\langle b, x\rangle$ and $\langle b, y\rangle$ are isomorphic to the 
2A-algebra, i.e., $(a \mid x)=(a \mid y)=(b \mid x)=(b \mid y)=2^{-5}$. Then the Griess subalgebra of $\langle a, b, x, y\rangle$ generated by $a, b, x$ and $y$ is 18-dimensional spanned by

$$
\begin{aligned}
A=\left\{u_{a, b}, u_{x, y},\right. & u_{a \circ x, b \circ y}, u_{a \circ x, b \circ z}, a, b, c=\tau_{a} b, x, y, z=\tau_{x} y, \\
& a \circ x, a \circ y, a \circ z, b \circ x, b \circ y, c \circ z, c \circ x, c \circ y, c \circ z\}
\end{aligned}
$$

with one linear relation

$u_{a, b}+u_{x, y}+u_{a \circ x, b \circ y}+u_{a \circ x, b \circ z}=\frac{32}{45}(a \circ x+a \circ y+a \circ z+b \circ x+b \circ y+c \circ z, c \circ x, c \circ y, c \circ z)$.

The conformal vector of $\langle a, b, x, y\rangle$ is

$$
\eta=\frac{17}{22}\left(u_{a, b}+u_{x, y}\right)+\frac{10}{11}\left(u_{a \circ x, b \circ y}+u_{a \circ x, b \circ z}\right)+\frac{16}{33}(a+b+c+x+y+z)
$$

and has central charge $228 / 55$. The VOA $\langle a, b, x, y\rangle$ has a full subVOA isomorphic to

$$
L\left(c_{3}, 0\right) \otimes L\left(c_{3}, 0\right) \otimes L\left(c_{3}, 0\right) \otimes L\left(c_{3}, 0\right) \otimes L\left(c_{8}, 0\right)=L(4 / 5,0)^{\otimes 4} \otimes L(52 / 55,0) .
$$

Proof: We have already shown that the Griess subalgebra generated by $a, b, x, y$ is linearly spanned by $A$. We also know the linear relation in Theorem 4.7. It is straightforward to verify that the determinant of the Gram matrix of $A \backslash\left\{u_{a \circ x, b \circ z}\right\}$ is equal to $3^{52} \cdot 11 \cdot 13^{6} / 2^{138} \cdot 5^{3}$. Therefore, the linear span of $A$ is 18 -dimensional. The conformal vector of $\langle a, b, x, y\rangle$ is given in Proposition 4.12 .

The subalgebra $\langle a \circ x, a \circ y, b \circ x\rangle$ is isomorphic to the ternary code VOA and has the Virasoro frame $u_{a, b}+u_{x, y}+u_{a \circ x, b \circ y}+u_{a \circ x, b \circ z}$. That is, $\langle a \circ x, a \circ y, b \circ x\rangle$ is a $\mathbb{Z}_{3} \oplus \mathbb{Z}_{3}$-graded simple current extension of $L(4 / 5,0)^{\otimes 4}$. Then the element

$$
\begin{aligned}
\xi & =\eta-\left(u_{a, b}+u_{x, y}+u_{a \circ x, b \circ y}+u_{a \circ x, b \circ z}\right) \\
& =\frac{5}{22}\left(u_{a, b}+u_{x, y}\right)+\frac{1}{11}\left(u_{a \circ x, b \circ y}+u_{a \circ x, b \circ z}\right)+\frac{16}{33}(a+b+c+x+y+z)
\end{aligned}
$$

is a Virasoro vector orthogonal to $u_{a, b}+u_{x, y}+u_{a \circ x, b \circ y}+u_{a \circ x, b \circ z}$. The central charge of $\xi$ is $228 / 55-4 \cdot 4 / 5=52 / 55=c_{8}$. Therefore, $\langle a, b, x, y\rangle$ has the full subVOA

$$
\langle a \circ x, a \circ y, b \circ x, \xi\rangle \cong\langle a \circ x, a \circ y, b \circ x\rangle \otimes\langle\xi\rangle
$$

which contains a unitary Virasoro frame $L(4 / 5,0)^{\otimes 4} \otimes L(52 / 55,0)$.

\subsection{Inductive subalgebras}

Let $a, b$ be Ising vectors of $V_{\mathbb{R}}$ such that $\langle a, b\rangle$ is isomorphic to the 3A-algebra, i.e., $(a \mid b)=13 \cdot 2^{-10}$ and let $I_{a, b}$ be defined as in (3.1). It is shown in Corollary 3.6 that $\left\langle\tau_{x} \mid x \in I_{a, b}\right\rangle$ is a 3-transposition group. In order to study the inductive structures and basic sets of this group, we will choose $x^{i} \in I_{a, b}$ and define subalgebras $X^{[i]}=\left\langle X^{[i-1]}, x^{i}\right\rangle$ inductively as follows. 
Definition 4.14. Set $X^{[0]}=\langle a, b\rangle$. Suppose we have chosen $x^{1}, \ldots, x^{i} \in I_{a, b}$ and defined

$$
X^{[i]}:=\left\langle a, b, x^{1}, \ldots, x^{i}\right\rangle \quad \text { for some } i \geq 0 .
$$

We choose $x^{i+1} \in I_{a, b}$ such that $x^{i+1} \notin X^{[i]}$ and $\left(x^{i+1} \mid x^{j}\right)=2^{-5}$ for all $1 \leq j \leq i$ and define the subalgebra $X^{[i+1]}$ by

$$
X^{[i+1]}:=\left\langle X^{[i]}, x^{i+1}\right\rangle=\left\langle a, b, x^{1}, \ldots, x^{i}, x^{i+1}\right\rangle
$$

as long as possible.

We will show that the structure of the Griess algebra of $X^{[n]}$ does not depend on the choice of $x^{i}, 1 \leq i \leq n$, and is uniquely determined up to isomorphism. First, we observe from [Ma05] that the Griess algebra of $\left\langle x^{1}, \ldots, x^{n}\right\rangle$ is uniquely determined by our choice.

Proposition 4.15 ([Ma05]). Suppose $x^{1}, \cdots, x^{n}$ are Ising vectors such that $\left(x^{i} \mid x^{j}\right)=2^{-5}$ and $x^{k} \notin\left\langle x^{1}, \ldots, x^{k-1}\right\rangle$ for any $1 \leq i<j \leq n$ and $1<k \leq n$. Then the Griess algebra of $\left\langle x^{1}, \ldots, x^{n}\right\rangle$ has a unique structure with a basis $\left\{x^{i}, x^{j} \circ x^{k} \mid 1 \leq i \leq n, 1 \leq j<k \leq n\right\}$.

Proof: Let $i, j, k$ be distinct. By Lemma 2.10, we have $\left(x^{i} \circ x^{j} \mid x^{k}\right)=0$ and $\left\langle x^{i} \circ x^{j}, x^{k}\right\rangle$ is the 2B-algebra. Therefore $x_{(1)}^{k}\left(x^{i} \circ x^{j}\right)=0$ and $\sigma_{x^{k}}$ fixes $x^{i} \circ x^{j}$. Then by conjugating $\left\{x^{i}, x^{j}, x^{i} \circ x^{j}\right\}$ by $\sigma_{x^{k}}$ we see that $\left\{x^{i} \circ x^{j}, x^{i} \circ x^{k}, x^{j} \circ x^{k}\right\}$ forms a 2A-triple. Moreover, if $l$ is distinct from $i, j$ and $k$, then

$$
\left(x^{i} \circ x^{j} \mid x^{k} \circ x^{l}\right)=\left(x^{i}+x^{j}-4 x_{(1)}^{i} x^{j} \mid x^{k} \circ x^{l}\right)=-4\left(x^{i} \mid x_{(1)}^{j}\left(x^{k} \circ x^{l}\right)\right)=0 .
$$

Therefore, $\left\langle x^{i} \circ x^{j}, x^{k} \circ x^{l}\right\rangle$ is a $2 \mathrm{~B}$-algebra. Hence, the Griess subalgebra generated by $x^{i}, 1 \leq i \leq n$, is uniquely determined and linearly spanned by Ising vectors $x^{i}, 1 \leq i \leq n$ and $x^{j} \circ x^{k}, 1 \leq j<k \leq n$. Such Ising vectors can be realized inside $V_{\sqrt{2} A_{n}}^{+}$and known to be linearly independent (cf. [DLMN98, LSY07, Ma01]). This completes the proof.

Remark 4.16. The Griess algebra of $\left\langle x^{1}, \ldots, x^{n}\right\rangle$ coincides with that of $M_{A_{n}}$ in [LSY07] (cf. Proposition A.3). Set $\tilde{x}^{1}=x^{1}$ and $\tilde{x}^{i}=x^{i} \circ x^{i-1}$ for $2 \leq i \leq n$. It is clear that $\left\langle x^{1}, \ldots, x^{n}\right\rangle=\left\langle\tilde{x}^{1}, \ldots, \tilde{x}^{n}\right\rangle$. We have $\left(\tilde{x}^{i} \mid \tilde{x}^{j}\right)=0$ if $|i-j|>1$ and $\left(\tilde{x}^{k} \mid \tilde{x}^{k+1}\right)=2^{-5}$ for $1 \leq k<n$. Then the associated involutions $\sigma_{\tilde{x}^{1}}, \ldots, \sigma_{\tilde{x}^{n}}$ acting on the subalgebra $\left\langle\tilde{x}^{1}, \ldots, \tilde{x}^{n}\right\rangle$ satisfy the Coxeter relation of type $A_{n}$ and hence $\left\langle\sigma_{\tilde{x}^{1}}, \ldots, \sigma_{\tilde{x}^{n}}\right\rangle$ is isomorphic to the symmetric group $\mathrm{S}_{n+1}$. If we identify this group as a permutation group of the $(n+1)$-set $\{0,1,2, \ldots, n\}$, then the involutions $\sigma_{x^{i}}$ and $\sigma_{x^{j} \circ x^{k}}=\sigma_{x^{j}} \sigma_{x^{k}} \sigma_{x^{j}}$ correspond to the transpositions $(0 i)$ and $(j k)$, respectively.

Theorem 4.17. With reference to the above, the Griess algebra generated by Ising vectors $a, b, x^{1}, \ldots, x^{n}$ is uniquely determined and has the following basis:

$$
u=u_{a, b}, a, b, c=\tau_{a} b, x^{i}, a \circ x^{i}, b \circ x^{i}, c \circ x^{i}, x^{j} \circ x^{k}, \quad 1 \leq i \leq n, \quad 1 \leq j<k \leq n .
$$


The conformal vector of $X^{[n]}=\left\langle a, b, x^{1}, \ldots, x^{n}\right\rangle$ is given by

$$
\begin{aligned}
\omega^{n} & =\frac{3(3-n)}{2(n+7)} u+\frac{16}{3(n+7)}\left(a+b+c+\sum_{i=1}^{n}\left(a \circ x^{i}+b \circ x^{i}+c \circ x^{i}\right)\right) \\
& +\frac{4}{n+7}\left(\sum_{i=1}^{n} x^{i}+\sum_{1 \leq j<k \leq n} x^{j} \circ x^{k}\right)
\end{aligned}
$$

and its central charge is equal to $(n+2)(5 n+29) / 5(n+7)$. The commutant $\operatorname{Com}_{X^{[n]}} X^{[n-1]}$ has the $c=c_{n+4}$ conformal vector

$$
f^{n}=\omega^{n}-\omega^{n-1}
$$

Therefore, $X^{[n]}$ has a unitary Virasoro frame $L\left(c_{3}, 0\right) \otimes L\left(c_{4}, 0\right) \otimes \cdots \otimes L\left(c_{n+4}, 0\right)$.

Proof: $\quad$ Set $c=\tau_{a} b=\tau_{b} a$ and

$$
A=\left\{u_{a, b}, a, b, c, x^{i}, a \circ x^{i}, b \circ x^{i}, c \circ x^{i}, x^{j} \circ x^{k} \mid 1 \leq i \leq n, \quad 1 \leq j<k \leq n\right\} .
$$

It follows from Lemma 3.2 and (1) of Proposition 4.2 that both $\left\langle a, b \circ x^{i}\right\rangle$ and $\left\langle a \circ x^{i}, b \circ x^{j}\right\rangle$ are isomorphic to the $6 \mathrm{~A}$-algebra. Therefore, we have the following subalgebras in the Griess algebra:

$$
\begin{aligned}
& \langle a, b\rangle_{2}=\operatorname{Span}\left\{u_{a, b}, a, b, c\right\}, \\
& \left\langle a, a \circ x^{i}\right\rangle_{2}=\left\langle a, x^{i}\right\rangle_{2}=\operatorname{Span}\left\{a, a \circ x^{i}, x^{i}\right\}, \\
& \left\langle a, b \circ x^{i}\right\rangle_{2}=\operatorname{Span}\left\{u_{a, b}, x^{i}, a, b, c, a \circ x^{i}, b \circ x^{i}, c \circ x^{i}\right\}, \\
& \left\langle a \circ x^{i}, b \circ x^{i}\right\rangle_{2}=\sigma_{x^{i}}\langle a, b\rangle_{2}=\operatorname{Span}\left\{u_{a, b}, a \circ x^{i}, b \circ x^{i}, c \circ x^{i}\right\}, \\
& \left\langle a \circ x^{i}, b \circ x^{j}\right\rangle_{2}=\operatorname{Span}\left\{u_{a, b}, x^{i} \circ x^{j}, a \circ x^{i}, b \circ x^{i}, c \circ x^{i}, a \circ x^{j}, b \circ x^{j}, c \circ x^{j}\right\} .
\end{aligned}
$$

In particular, the following orthogonality relation holds.

$$
\left(u_{a, b} \mid x^{i}\right)=\left(u_{a, b} \mid x^{i} \circ x^{j}\right)=0 .
$$

Applying Proposition 4.15 to the subalgebra $\left\langle a, x^{1}, \ldots, x^{n}\right\rangle$, we also have the following orthogonality.

$$
\left(a \mid x^{j} \circ x^{k}\right)=\left(a \circ x^{i} \mid x^{j}\right)=\left(x^{i} \mid x^{j} \circ x^{k}\right)=\left(a \circ x^{i} \mid x^{j} \circ x^{k}\right)=0 .
$$

On $X^{[n]}$, all $\tau_{x^{i}}$ and $\tau_{x^{i} \circ x^{j}}$ are trivial and we have $\tau_{e o x^{i}}=\tau_{e}$ for $e \in\{a, b, c\}$ by (5) of Theorem 2.9. Then $\left\langle\tau_{a}, \tau_{b}\right\rangle \cong \mathrm{S}_{3}$ acts faithfully on each of the 3 -sets $\left\{a \circ x^{i}, b \circ x^{i}, c \circ x^{i}\right\}, 1 \leq$ $i \leq n$. The group generated by $\sigma_{x^{i}}, 1 \leq i \leq n$, is isomorphic to $\mathrm{S}_{n+1}$ and this group acts transitively on the set of Ising vectors $\left\{x^{i}, x^{j} \circ x^{k} \mid 1 \leq i \leq n, 1 \leq j<k \leq n\right\}$ (cf. Remark 
4.16). Set $G=\left\langle\tau_{a}, \tau_{b}, \sigma_{x^{1}}, \ldots, \sigma_{x^{n}}\right\rangle \subset \operatorname{Aut}\left(X^{[n]}\right)$. Then $A$ is $G$-invariant and the products and inner products of vectors in $A$ are uniquely determined in the linear span of $A$. Therefore, the linear span of $A$ forms a subalgebra in the Griess algebra. That $A$ is linearly independent will be shown in Appendix A.3 using an explicit construction.

Next we prove that $\omega^{n}$ is the conformal vector of $X^{[n]}$. It is clear that $\omega^{n}$ is fixed by $G$. By using $G$-invariance, one can directly verify that $\omega^{n}$ satisfies $\left(\omega^{n} \mid t\right)=(t \mid t)$ for all $t \in A$. Therefore, $\omega^{n}$ is the conformal vector of $X^{[n]}$ by Lemma 4.3. The central charge of $\omega^{n}$ is $2\left(\omega^{n} \mid \omega^{n}\right)=(n+2)(5 n+29) / 5(n+7)$. Since $X^{[n-1]}$ is a subalgebra of $X^{[n]}$, both $\omega^{n-1}$ and $f^{n}=\omega^{n}-\omega^{n-1}$ are mutually orthogonal Virasoro vectors of $X^{[n]}$ and $f^{n}$ is the conformal vector of $\operatorname{Com}_{X^{[n]}} X^{[n-1]}$ by Theorem 5.1 of [FZ92]. The central charge of $f^{n}$ is

$$
2\left(\omega^{n} \mid \omega^{n}\right)-2\left(\omega^{n-1} \mid \omega^{n-1}\right)=\frac{n^{2}+13 n+36}{(n+6)(n+7)}=c_{n+4} .
$$

Set

$$
v_{a, b}=-\frac{5}{14} u_{a, b}+\frac{16}{21}(a+b+c) .
$$

Then $v_{a, b}$ is a $c=c_{4}=6 / 7$ Virasoro vector and the conformal vector $\omega^{0}$ of $X^{[0]}=\langle a, b\rangle$ is an orthogonal sum $\omega^{0}=u_{a, b} \dot{+} v_{a, b}$ by (6) of Theorem 2.11. Therefore, we have the following orthogonal decompositions:

$$
\omega^{n}=\omega^{0} \dot{+} f^{1} \dot{+} \cdots \dot{+} f^{n}=u_{a, b} \dot{+} v_{a, b} \dot{+} f^{1} \dot{+} \cdots \dot{+} f^{n} .
$$

This shows $X^{[n]}$ has a full subVOA isomorphic to $L\left(c_{3}, 0\right) \otimes L\left(c_{4}, 0\right) \otimes \cdots \otimes L\left(c_{n+4}, 0\right)$. This completes the proof.

Set $D^{[0]}=\left\{\tau_{x} \in \operatorname{Aut}(V) \mid x \in I_{a, b}\right\}$ and inductively we define

$$
D^{[i]}:=\left\{\tau_{y} \in D^{[i-1]} \mid \tau_{y} \tau_{x^{i}}=\tau_{x^{i}} \tau_{y}\right\}
$$

It is clear that $\left\langle D^{[i]}\right\rangle$ is a subgroup of the centralizer of $\left\langle\tau_{a}, \tau_{b}, \tau_{x^{1}}, \cdots, \tau_{x^{n}}\right\rangle$ in $\operatorname{Aut}(V)$. For inductive arguments in the next section, we will consider the action of $\left\langle D^{[i]}\right\rangle$ restricted to a smaller subalgebra.

Lemma 4.18. Each involution in $D^{[n]}$ acts trivially on $X^{[n]}$.

Proof: We prove this by induction on $n$. By definition, both $\langle a, x\rangle$ and $\langle b, x\rangle$ are 2Aalgebras for each $x \in D^{[0]}=I_{a, b}$, and it follows that $\tau_{x}$ fixes both $a$ and $b$. Thus $D^{[0]}$ acts trivially on $X^{[0]}=\langle a, b\rangle$. Suppose each involution of $D^{[i]}$ acts on $X^{[i]}=\left\langle a, b, x^{1}, \cdots, x^{i}\right\rangle$ trivially. Let $\tau_{y} \in D^{[i+1]}$. Then $\tau_{y} \in D^{[i]}$ and $\tau_{y}$ acts trivially on $X^{[i]}$. Since $\tau_{y}$ and $\tau_{x^{i+1}}$ commute, it follows from Theorem 2.6 that $\left\langle y, x^{i+1}\right\rangle$ is a dihedral algebra of type $1 \mathrm{~A}, 2 \mathrm{~A}$ or $2 \mathrm{~B}$. In each case $\tau_{y}$ fixes $x^{i+1}$. Therefore, $\tau_{y}$ acts trivially on $X^{[i+1]}=\left\langle X^{[i]}, x^{i+1}\right\rangle$. 
By this lemma, we have a group homomorphism by restriction.

$$
\begin{aligned}
\varphi^{[i]}:\left\langle D^{[i]}\right\rangle & \longrightarrow \operatorname{Aut}\left(\operatorname{Com}_{V} X^{[i]}\right) \\
g & \left.\longmapsto g\right|_{\operatorname{Com}_{V} X^{[i]}}
\end{aligned}
$$

Set $G^{[i]}:=\operatorname{Im} \varphi^{[i]} \subset \operatorname{Aut}\left(\operatorname{Com}_{V} X^{[i]}\right)$. It follows from Corollary 3.6 that $\left(G^{[i]}, \varphi^{[i]}\left(D^{[i]}\right)\right)$

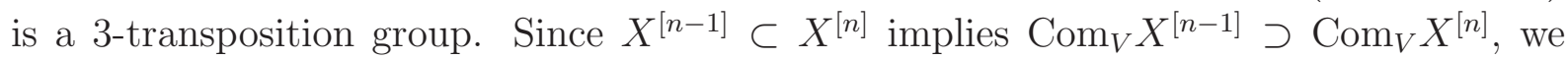
obtain the following inductive structure:

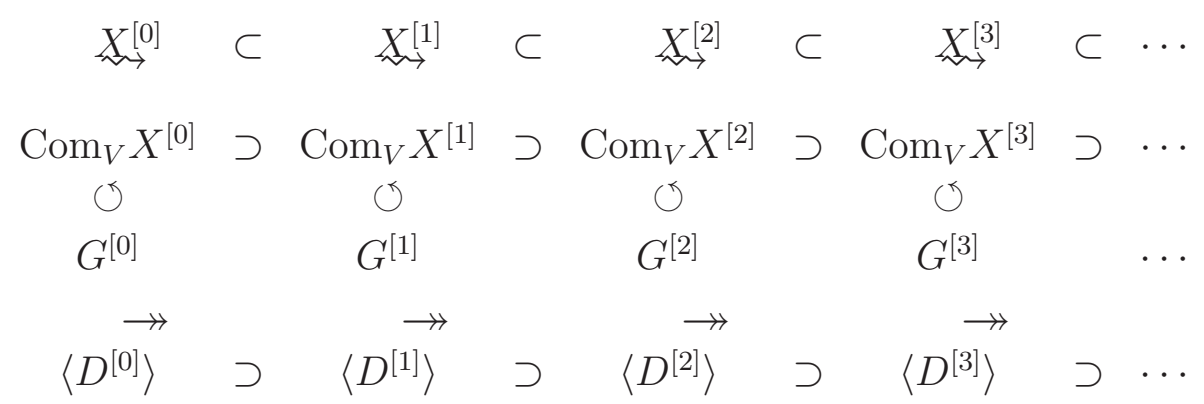

Note that $\varphi^{[n-1]}\left(\tau_{x^{n}}\right) \in G^{[n-1]}$ is an external automorphism of $\mathrm{Com}_{V} X^{[n-1]}$ in the sense that $x^{n} \notin \mathrm{Com}_{V} X^{[n-1]}$. By Theorem 5.2 of [FZ92] and (4.18) we have

$$
\operatorname{Com}_{V} X^{[n]}=\operatorname{Com}_{V}\left\langle u_{a, b}, v_{a, b}, f^{1}, \ldots, f^{n}\right\rangle .
$$

Since the decomposition in (4.18) is orthogonal, we have $f^{n} \in \operatorname{Com}_{V} X^{[n-1]}$. We prove that $\varphi^{[n-1]}\left(\tau_{x^{n}}\right)$ can be described as an internal automorphism defined by $f^{n}$.

Theorem 4.19. Let $X^{[n]}=\left\langle a, b, x^{1}, \ldots, x^{n}\right\rangle$ be the subalgebra of a VOA $V$ defined by (4.13) and let $\omega^{n}=u_{a, b} \dot{+} v_{a, b} \dot{+} f^{1} \dot{+} \cdots \dot{+} f^{n}$ be the orthogonal decomposition of the conformal vector of $X^{[n]}$ as in (4.18).

(1) If $n$ is odd, then $\tau_{f^{n}}=\tau_{x^{1}} \tau_{x^{2}} \cdots \tau_{x^{n}}$ in $\operatorname{Aut}(V)$ and $\tau_{f^{n}}=\varphi^{[n-1]}\left(\tau_{x^{n}}\right)$ as an automorphism of $\operatorname{Com}_{V} X^{[n-1]}$.

(2) If $n>0$ is even, then $\tau_{f^{n}}=\tau_{f^{n-1}}=\tau_{x^{1}} \tau_{x^{2}} \cdots \tau_{x^{n-1}}$ in $\operatorname{Aut}(V)$ and $\varphi^{[n-1]}\left(\tau_{f^{n}}\right)$ is trivial in $\operatorname{Aut}\left(\mathrm{Com}_{V} X^{[n-1]}\right)$. In this case, $f^{n}$ is of $\sigma$-type on $\operatorname{Com}_{V} X^{[n-1]}$ and satisfies $\sigma_{f^{n}}=\varphi^{[n-1]}\left(\tau_{x^{n}}\right)$ as an automorphism of $\operatorname{Com}_{V} X^{[n-1]}$.

Proof: By direct computations, we have the following relations in $X^{[n]}$.

$$
\begin{aligned}
& a_{(1)} u_{a, b}=\frac{2}{3} a+\frac{5}{24} u_{a, b}-\frac{7}{24} v_{a, b}, \quad a_{(1)} v_{a, b}=\frac{4}{3} a-\frac{5}{24} u_{a, b}+\frac{7}{24} v_{a, b}, \\
& x_{(1)}^{1} v_{a, b}=\frac{5}{7} x^{1}+\frac{3}{14} v_{a, b}-\frac{2}{7} f^{1}, \quad x_{(1)}^{1} f^{1}=\frac{9}{7} x^{1}-\frac{3}{14} v_{a, b}+\frac{2}{7} f^{1}, \\
& \left(x^{i} \circ x^{i+1}\right)_{(1)} f^{i}=\frac{i+5}{i+7} x^{i} \circ x^{i+1}+\frac{i+6}{4(i+7)} f^{i}-\frac{i+8}{4(i+7)} f^{i+1}, \\
& \left(x^{i} \circ x^{i+1}\right)_{(1)} f^{i+1}=\frac{i+9}{i+7} x^{i} \circ x^{i+1}-\frac{i+6}{4(i+7)} f^{i}+\frac{i+8}{4(i+7)} f^{i+1},
\end{aligned}
$$


where $1 \leq i \leq n-1$. By these relations, it follows from Proposition 4.2 of [LY16] that the subalgebras $\left\langle a, u_{a, b}, v_{a, b}\right\rangle,\left\langle x^{1}, v_{a, b}, f^{1}\right\rangle$ and $\left\langle x^{i} \circ x^{i+1}, f^{i}, f^{i+1}\right\rangle$ are uniquely determined and isomorphic to $A\left(1 / 2, c_{3}^{1}\right), A\left(1 / 2, c_{4}^{1}\right)$ and $A\left(1 / 2, c_{i+4}^{1}\right)$ in (loc. cit.), respectively. Then by Theorem 4.6 of [LY16], we have the following relations in $\operatorname{Aut}(V)$.

$$
\tau_{u_{a, b}}=\tau_{v_{a, b}}, \quad \tau_{v_{a, b}} \tau_{f^{1}}=\tau_{x^{1}}, \quad \tau_{f^{i}} \tau_{f^{i+1}}= \begin{cases}\tau_{x^{i} \circ x^{i+1}}=\tau_{x^{i}} \tau_{x^{i+1}} & \text { if } i \text { is even } \\ 1 & \text { if } i \text { is odd }\end{cases}
$$

It is also shown in Remark 2.12 of [HLY12b] that $\tau_{u_{a, b}}=\tau_{v_{a, b}}$ is trivial on $V$. Therefore, $\tau_{f^{1}}=\tau_{x^{1}}$ and by induction we have

$$
\tau_{f^{n}}= \begin{cases}\tau_{x^{1}} \tau_{x^{2}} \cdots \tau_{x^{n}} & \text { if } n \text { is odd } \\ \tau_{x^{1}} \tau_{x^{2}} \cdots \tau_{x^{n-1}} & \text { if } n>0 \text { is even }\end{cases}
$$

It is clear that $\tau_{x^{i}}$ are trivial on the commutant $\operatorname{Com}_{V} X^{[n-1]}$ for $1 \leq i \leq n-1$ since $x^{1}, \ldots, x^{n-1} \in X^{[n-1]}$. Thus as an automorphism of $\operatorname{Com}_{V} X^{[n-1]}$ we have

$$
\tau_{f^{n}}= \begin{cases}\varphi^{[n-1]}\left(\tau_{x^{1}} \cdots \tau_{x^{n}}\right)=\varphi^{[n-1]}\left(\tau_{x^{n}}\right) & \text { if } n \text { is odd } \\ \varphi^{[n-1]}\left(\tau_{x^{1}} \cdots \tau_{x^{n-1}}\right)=1 & \text { if } n>0 \text { is even }\end{cases}
$$

If $n>0$ is even, it is shown in Theorem 4.8 of [LY16] that $f^{n}$ is of $\sigma$-type on the commutant $\operatorname{Com}_{V}\left\langle f^{n-1}\right\rangle$ and both $\sigma_{f^{n}}$ and $\tau_{x^{n-1} \circ x^{n}}$ define the same automorphism on $\operatorname{Com}_{V}\left\langle f^{n-1}\right\rangle$. Since $\operatorname{Com}_{V} X^{[n-1]} \subset \operatorname{Com}_{V}\left\langle f^{n-1}\right\rangle$, we have

$$
\sigma_{f^{n}}=\varphi^{[n-1]}\left(\tau_{x^{n-1} \circ x^{n}}\right)=\varphi^{[n-1]}\left(\tau_{x^{n-1}} \tau_{x^{n}}\right)=\varphi^{[n-1]}\left(\tau_{x^{n}}\right)
$$

as an automorphism of $\operatorname{Com}_{V} X^{[n-1]}$. This completes the proof.

\section{Conway-Miyamoto correspondence}

In this section, we apply our theorems to the moonshine vertex operator algebra and establish the Conway-Miyamoto correspondence for the Fischer 3-transposition groups.

Throughout this section, we identify the Monster simple group $\mathbb{M}$ with the automorphism group of the moonshine VOA $V^{\natural}$ (cf. [FLM88]). The moonshine VOA $V^{\natural}$ can be defined over the real numbers and has a compact real form $V_{\mathbb{R}}^{\natural}$ as in [FLM88, Mi04]. It is shown in Proposition 5.2 of [HLY12a] that all Ising vectors in $V^{\natural}$ are contained in the compact real form $V_{\mathbb{R}}^{\natural}$. Therefore, $V^{\natural}$ has a canonical real form defined as the real subalgebra generated by Ising vectors, which indeed coincides with $V_{\mathbb{R}}^{\natural}$. In the following discussion, we will consider subalgebras generated by Ising vectors of $V^{\natural}$ so that all subalgebras have compact real forms. 
Definition 5.1. Let $V$ be a VOA and $G$ a subgroup of Aut $(V)$. Let $I$ be a conjugacy class of involutions of $G$. We define the Conway-Miyamoto correspondence between involutions $I$ of $G$ and $c=c_{n}$ Virasoro vectors of $V$ by means of the following conditions:

(1) For each $t \in I$, there exists a unique $c=c_{n}$ Virasoro vector $e_{t} \in V^{C_{G}(t)}$.

(2) If the unique Virasoro vector $e_{t}$ is not of $\sigma$-type on $V$, then $\tau_{e_{t}}=t$ on $V$. Otherwise, $\sigma_{e_{t}}=t$ on $V$.

The unique $c=c_{n}$ Virasoro vector $e_{t}$ of $V^{C_{G}(t)}$ is called the axial vector associated to $t$. We say that the Conway-Miyamoto correspondence between $G$ and $V$ is bijective if the axial vector $e_{t}$ is the unique $c=c_{n}$ Virasoro vector $a$ of $V$ satisfying $\tau_{a}=t$ (or $\sigma_{a}=t$ in the case $e_{t}$ is of $\sigma$-type on $V$ ).

Here are known examples of Conway-Miyamoto correspondences.

Theorem 5.2 ([ATLAS, FLM88, C85, Mi96, Ma01, H10, HLY12a, HLY12b]). There are Conway-Miyamoto correspondences between the following groups and VOAs. (1) 2A-elements of the Monster and Ising vectors of $V^{\natural}$. The correspondence is bijective. (2) 2A-elements of the Baby Monster and $c=c_{2}$ Virasoro vectors of $\sigma$-type of $V B^{\natural}=$ $\mathrm{Com}_{V^{\natural}}\langle e\rangle$, where $e$ is an Ising vector of $V^{\natural}$. The correspondence is bijective.

(3) $2 C$-elements of the largest Fischer 3-transposition group and $c=c_{4}$ Virasoro vectors of $\sigma$-type of $V F^{\natural}=\operatorname{Com}_{V^{\natural}}\left\langle u_{a, b}\right\rangle$, where $a, b$ are Ising vectors of $V^{\natural}$ such that $(a \mid b)=13 \cdot 2^{-10}$.

By the bijective correspondence in (1) of Theorem 5.2 and Proposition 2.5, we have

Corollary 5.3. Let $e^{1}, \ldots, e^{k}$ be Ising vectors of $V^{\natural}$. Then the centralizer $C_{\mathbb{M}}\left(\tau_{e^{1}}, \cdots, \tau_{e^{k}}\right)$ coincides with the pointwise stabilizer of $\left\langle e^{1}, \ldots, e^{k}\right\rangle$ in $\mathbb{M}$.

Now we fix a pair of Ising vectors $a, b$ in $V^{\natural}$ such that $\langle a, b\rangle$ is isomorphic to the $3 \mathrm{~A}$-algebra. Such a pair corresponds to a pair of $2 \mathrm{~A}$-involutions $\tau_{a}$ and $\tau_{b}$ such that $\tau_{a} \tau_{b}$ is a 3A-element by (1) of Theorem 5.2, and is known to be unique up to conjugation by $\mathbb{M}$ (cf. [ATLAS]).

Let $E_{V^{\natural}}$ be the set of Ising vectors of $V^{\natural}$ and $I_{a, b}=\left\{x \in E_{V^{\natural}} \mid(a \mid x)=(b \mid x)=2^{-5}\right\}$ as before. For $n=0,1,2$, we consider the subalgebra $X^{[n]}=\left\langle a, b, x^{1}, \cdots, x^{n}\right\rangle$ of $V^{\natural}$ defined as in Sec. 4.3 and study the automorphism group $G^{[n]}=\left\langle\varphi^{[i]}\left(D^{[i]}\right)\right\rangle$ of $\operatorname{Com}_{V^{\natural}} X^{[n]}$ defined as in (4.19) and (4.20).

Proposition 5.4. Let $a, b, x^{1}, x^{2} \in V^{\natural}$ be as above.

(1) $\left\langle D^{[0]}\right\rangle=C_{\mathbb{M}}\left(\tau_{a}, \tau_{b}\right) \cong \mathrm{Fi}_{23}$ in $\operatorname{Aut}\left(V^{\natural}\right)$ and $G^{[0]} \cong \mathrm{Fi}_{23}$ in $\operatorname{Aut}\left(\operatorname{Com}_{V^{\natural}} X^{[0]}\right)$. Moreover, for any $\tau_{y} \in D^{[0]}, \varphi^{[0]}\left(\tau_{y}\right)$ defines a 2A-element of $\mathrm{Fi}_{23}$ on $\operatorname{Aut}\left(\mathrm{Com}_{V^{\natural}} X^{[0]}\right)$.

(2) $\left\langle D^{[1]}\right\rangle=C_{\mathbb{M}}\left(\tau_{a}, \tau_{b}, \tau_{x^{1}}\right) \cong 2 . \mathrm{Fi}_{22}$ in $\operatorname{Aut}\left(V^{\natural}\right)$ and $G^{[1]} \cong \mathrm{Fi}_{22}$ in $\operatorname{Aut}\left(\operatorname{Com}_{V^{\natural}} X^{[1]}\right)$. Moreover, for any $\tau_{y} \in D^{[1]}, \varphi^{[1]}\left(\tau_{y}\right)$ defines a $2 A$-element of $\mathrm{Fi}_{22}$ on $\operatorname{Aut}\left(\mathrm{Com}_{V^{\natural}} X^{[1]}\right)$. 
(3) $\left\langle D^{[2]}\right\rangle=C_{\mathbb{M}}\left(\tau_{a}, \tau_{b}, \tau_{x^{1}}, \tau_{x^{2}}\right) \cong 2^{2} \cdot \operatorname{PSU}_{6}(2)$ and $G^{[2]} \cong \operatorname{PSU}_{6}(2)$ in $\operatorname{Aut}\left(\operatorname{Com}_{V^{\natural}} X^{[2]}\right)$. Moreover, for any $\tau_{y} \in D^{[2]}, \varphi^{[2]}\left(\tau_{y}\right)$ defines a $2 A$-element of $\operatorname{PSU}_{6}(2)$ on $\operatorname{Aut}\left(\operatorname{Com}_{V^{\natural}} X^{[2]}\right)$.

Proof: First, we observe that $\left\langle D^{[n]}\right\rangle$ is a normal subgroup of $C_{\mathbb{M}}\left(\tau_{a}, \tau_{b}, \tau_{x^{1}}, \ldots, \tau_{x^{n}}\right)$ by Proposition 2.5 .

(1): Since $\mathbb{M}$ acts transitively on pairs of Ising vectors of $V^{\natural}$ generating $6 \mathrm{~A}$-subalgebras [ATLAS], there exists a 6A-subalgebra of $V^{\natural}$ containing $\langle a, b\rangle$ [LM06] and its central Ising vector belongs to $I_{a, b}$ so that $D^{[0]}$ is non-trivial. Since $\left\langle D^{[0]}\right\rangle$ is a normal subgroup of $C_{\mathbb{M}}\left(\tau_{a}, \tau_{b}\right)$ and $C_{\mathbb{M}}\left(\tau_{a}, \tau_{b}\right) \cong \mathrm{Fi}_{23}$ is a simple group by [ATLAS], we have $\left\langle D^{[0]}\right\rangle=$ $C_{\mathbb{M}}\left(\tau_{a}, \tau_{b}\right) \cong \mathrm{Fi}_{23}$. By definition, $G^{[0]}$ is a homomorphic image of $\left\langle D^{[0]}\right\rangle$ and we have $G^{[0]} \cong \mathrm{Fi}_{23}$ in $\operatorname{Aut}\left(\mathrm{Com}_{V^{\natural}} X^{[0]}\right)$. By Corollary 3.6, $D^{[0]}$ is the set of 3-transpositions, and only the 2A-involutions satisfy the 3 -transposition property in $\mathrm{Fi}_{23}$ (cf. [ATLAS], [I76, Page 45]). Therefore, each involution in $D^{[0]}$ defines a $2 \mathrm{~A}$-element of $\mathrm{Fi}_{23}$.

(2): By (1) and Corollary 5.3 we have $C_{\mathbb{M}}\left(\tau_{a}, \tau_{b}, \tau_{x^{1}}\right) \cong C_{\mathrm{Fi}_{23}}(2 \mathrm{~A}) \cong 2 . \mathrm{Fi}_{22}$ [ATLAS]. Since $\left\langle D^{[1]}\right\rangle$ is normal in $C_{\mathbb{M}}\left(\tau_{a}, \tau_{b}, \tau_{x^{1}}\right)$, we have $\left\langle D^{[1]}\right\rangle=C_{\mathbb{M}}\left(\tau_{a}, \tau_{b}, \tau_{x^{1}}\right) \cong 2 . \mathrm{Fi}_{22}$ in $\operatorname{Aut}\left(V^{\natural}\right)$ where the center is generated by $\tau_{x^{1}}$. Then $G^{[1]} \cong \mathrm{Fi}_{22}$ since the center $\tau_{x^{1}}$ acts trivially on Aut $\left(\mathrm{Com}_{V^{\natural}} X^{[1]}\right)$ by Lemma 4.18. Again it follows from Corollary 3.6 that $\left\langle\varphi^{[1]}\left(D^{[1]}\right)\right\rangle$ is the set of 3-transpositions in $G^{[1]} \cong \mathrm{Fi}_{22}$ and only 2 A-involutions satisfy the 3-transposition property in $\mathrm{Fi}_{22}$ (cf. [ATLAS], [I76, Page 45]). Therefore $\varphi^{[1]}\left(\tau_{y}\right)$ defines a $2 \mathrm{~A}$-element of $\mathrm{Fi}_{22}$ on $\mathrm{Com}_{V^{\natural}} X^{[1]}$ for any $\tau_{y} \in D^{[1]}$.

(3): By (2) and Corollary 5.3, we have $\left\langle D^{[2]}\right\rangle=C_{\mathbb{M}}\left(\tau_{a}, \tau_{b}, \tau_{x^{1}}, \tau_{x^{2}}\right) \cong 2^{2} \cdot \operatorname{PSU}_{6}(2)$ [ATLAS]. Since the center $\left\langle\tau_{x^{1}}, \tau_{x^{2}}\right\rangle$ acts trivially on $\mathrm{Com}_{V^{\natural}} X^{[2]}$, we have $G^{[2]} \cong \operatorname{PSU}_{6}(2)$ in $\operatorname{Aut}\left(\mathrm{Com}_{V^{\natural}} X^{[2]}\right)$. Since only $2 \mathrm{~A}$-involutions satisfy the 3 -transposition property in $\mathrm{PSU}_{6}(2)$ (cf. [ATLAS], [I76, Page 45]), $\varphi^{[2]}\left(\tau_{y}\right)$ is a $2 \mathrm{~A}$-element for any $\tau_{y} \in D^{[2]}$. This completes the proof.

Lemma 5.5. Let $\omega^{0}, \omega^{1}$ and $\omega^{2}$ be the conformal vectors of the subalgebras $X^{[0]}=\langle a, b\rangle$, $X^{[1]}=\left\langle a, b, x^{1}\right\rangle$ and $X^{[2]}=\left\langle a, b, x^{1}, x^{2}\right\rangle$ of $V^{\natural}$, respectively, and let $f^{1}=\omega^{1}-\omega^{0}$ and $f^{2}=\omega^{2}-\omega^{1}$ be the conformal vectors of $\operatorname{Com}_{X^{[1]}} X^{[0]}$ and $\operatorname{Com}_{X^{[2]}} X^{[1]}$, respectively. Then $f^{1}$ is fixed by $C_{G^{[0]}}\left(\varphi^{[0]}\left(\tau_{x^{1}}\right)\right)$ and $f^{2}$ is fixed by $C_{G^{[1]}}\left(\varphi^{[1]}\left(\tau_{x^{2}}\right)\right)$.

Proof: It follows from Proposition 5.4 that $C_{G^{[0]}}\left(\varphi^{[0]}\left(\tau_{x^{1}}\right)\right)=\varphi^{[0]}\left(C_{\mathbb{M}}\left(\tau_{a}, \tau_{b}, \tau_{x^{1}}\right)\right)$ and $C_{G^{[1]}}\left(\varphi^{[1]}\left(\tau_{x^{2}}\right)\right)=\varphi^{[1]}\left(C_{\mathbb{M}}\left(\tau_{a}, \tau_{b}, \tau_{x^{1}}, \tau_{x^{2}}\right)\right)$. Then $C_{\mathbb{M}}\left(\tau_{a}, \tau_{b}, \tau_{x^{1}}\right)$ fixes $f^{1}=\omega^{0}-\omega^{1} \in$ $\left\langle a, b, x^{1}\right\rangle$ and $C_{\mathbb{M}}\left(\tau_{a}, \tau_{b}, \tau_{x^{1}}, \tau_{x^{2}}\right)$ fixes $f^{2}=\omega^{1}-\omega^{2} \in\left\langle a, b, x^{1}, x^{2}\right\rangle$ by Corollary 5.3. Thus the claim follows. 


\subsection{Transpositions of $\mathrm{Fi}_{23}$ and $c=25 / 28$ Virasoro vectors}

Let $a, b$ be Ising vectors of $V^{\natural}$ such that $\langle a, b\rangle$ is the 3A-algebra. In this subsection, we consider $X^{[0]}=\langle a, b\rangle \subset V^{\natural}$ and its commutant subalgebra $V F_{23}^{\natural}=\operatorname{Com}_{V^{\natural}}\langle a, b\rangle$. Let

$$
D^{[0]}=\left\{\tau_{x} \in \operatorname{Aut}\left(V^{\natural}\right) \mid x \in I_{a, b}\right\}, \quad G^{[0]}=\left\langle\varphi^{[0]}\left(D^{[0]}\right)\right\rangle \subset \operatorname{Aut}\left(\operatorname{Com}_{V^{\natural}}\langle a, b\rangle\right)
$$

be defined as in (4.19) and (4.20). We have shown in Proposition 5.4 that $G^{[0]} \cong \mathrm{Fi}_{23}$. In the following, we will identify $\mathrm{Fi}_{23}$ with $G^{[0]} \subset \operatorname{Aut}\left(\operatorname{Com}_{V^{\natural}}\langle a, b\rangle\right)$. Let $x^{1} \in D^{[0]}$ and $X^{[1]}=\left\langle a, b, x^{1}\right\rangle$. Let $f^{1}$ be the conformal vector of $\operatorname{Com}_{X^{[1]}} X^{[0]}$. Then it follows from Theorem 4.19 and Proposition 5.4 that $\tau_{f^{1}}=\varphi^{[0]}\left(\tau_{x^{1}}\right)$ defines a 2A-element of $\mathrm{Fi}_{23}$. Moreover, it is shown in Lemma 5.5 that $f^{1}$ is fixed by the centralizer $C_{\mathrm{Fi}_{23}}\left(\tau_{f^{1}}\right)$. We will show that $f^{1}$ is the unique $C_{\mathrm{Fi}_{23}}\left(\tau_{f^{1}}\right)$-invariant $c=c_{5}$ Virasoro vector of $\operatorname{Com}_{V^{\natural}}\langle a, b\rangle$.

Recall that the $3 \mathrm{~A}$-algebra $U_{3 \mathrm{~A}}=\langle a, b\rangle$ has 6 irreducible representations $U(h)$ which are distinguished by the top weights $h \in\{0,1 / 7,5 / 7,2 / 5,19 / 35,4 / 35\}$ (cf. [SY03, LLY03]). We denote by $\operatorname{Irr} U_{3 \mathrm{~A}}$ the set of equivalent classes of irreducible $U_{3 \mathrm{~A}}$-modules. For $U(h) \in$ $\operatorname{Irr} U_{3 \mathrm{~A}}$, we set

$$
U(h)^{c}:=\operatorname{Hom}_{\langle a, b\rangle}\left(U(h), V^{\natural}\right) .
$$

Then we have the isotypical decomposition

$$
V^{\natural}=\bigoplus_{U(h) \in \operatorname{Irr}\langle a, b\rangle} U(h) \otimes U(h)^{c} .
$$

The commutant $\operatorname{Com}_{V^{\natural}}\langle a, b\rangle$ acts naturally on $U(h)^{c}$ and one can consider (5.1) as the decomposition as a $\langle a, b\rangle \otimes \operatorname{Com}_{V^{\natural}}\langle a, b\rangle$-module. We denote the top levels of $U(h)$ and $U(h)^{c}$ by Top $U(h)$ and Top $U(h)^{c}$, respectively. The top weight and the dimension of the top level of $U(h)^{c}$ are obtained in Lemma 5.6 of [HLY12b].

Lemma 5.6. The top levels of $U(h)^{c}$ are irreducible as $G^{[0]} \cong \mathrm{Fi}_{23}$-modules and their characters are as in the following table.

\begin{tabular}{|c||c|c|c|c|c|c|}
\hline$U(h)$ & $U(0)$ & $U(5 / 7)$ & $U(19 / 35)$ & $U(2 / 5)$ & $U(1 / 7)$ & $U(4 / 35)$ \\
\hline $\operatorname{dim}$ Top $U(h)$ & 1 & 3 & 3 & 1 & 1 & 2 \\
\hline Top weight of $U(h)^{c}$ & 0 & $9 / 7$ & $51 / 35$ & $8 / 5$ & $13 / 7$ & $66 / 35$ \\
\hline $\operatorname{dim}$ Top $U(h)^{c}$ & 1 & 782 & 3588 & 5083 & 25806 & 60996 \\
\hline $\mathrm{Fi}_{23}$-character & $\chi_{1}$ & $\chi_{2}$ & $\chi_{3}$ & $\chi_{4}$ & $\chi_{5}$ & $\chi_{7}$ \\
\hline
\end{tabular}

Here $\chi_{i}$ denotes the irreducible characters of $F i_{23}$ labeled as in [ATLAS, page 178]. By abuse of notations, we also use $\chi_{i}$ to denote the irreducible module affording the character $\chi_{i}$. Then we have the decomposition $\left(\mathrm{Com}_{V \sharp}\langle a, b\rangle\right)_{2}=\chi_{1}+\chi_{6}(=\mathbf{1}+\mathbf{3 0 8 8 8})$ as a $\mathrm{Fi}_{23^{-}}$ module. 
The centralizer $C_{\mathrm{Fi}_{23}}\left(\tau_{f^{1}}\right)$ is isomorphic to $2 . \mathrm{Fi}_{22}$ by Proposition 5.4 and we have the following decomposition (cf. [ATLAS]).

Proposition 5.7. Consider the action of the centralizer $C_{G^{[0]}}\left(\varphi^{[0]}\left(\tau_{x^{1}}\right)\right) \cong 2 . \mathrm{Fi}_{22}$ on the Griess algebra of $\mathrm{Com}_{V^{\natural}}\langle a, b\rangle$ and the top levels $\operatorname{Top} U(h)^{c}$ of $U(h)^{c}$ in (5.1). As 2. $\mathrm{Fi}_{22^{-}}$ modules, we have the following decompositions.

$$
\begin{aligned}
& \left(\mathrm{Com}_{V^{\natural}}\langle a, b\rangle\right)_{2}=\underset{\chi_{1}}{\mathbf{1}}+\underset{\chi_{1}}{\mathbf{1}}+\underset{\chi_{3}}{\mathbf{4 2 9}}+\underset{\chi_{7}}{3080}+\underset{\chi_{9}}{13650}+\underset{\chi_{73}}{13728}, \\
& \text { Top } U(5 / 7)^{c}=\underset{\chi_{1}}{1}+\underset{\chi_{3}}{429}+\underset{\chi_{66}}{352} \\
& \text { Top } U(19 / 35)^{c}=\underset{\chi_{2}}{78}+\underset{\chi_{5}}{\mathbf{1 4 3 0}}+\underset{\chi_{68}}{\mathbf{2 0 8 0}} \\
& \text { Top } U(2 / 5)^{c}=\underset{\chi_{6}}{3003}+\underset{\chi_{67}}{2080} \\
& \text { Top } U(1 / 7)^{c}=\underset{\chi_{4}}{1001}+\underset{\chi_{8}}{10725}+\underset{\chi_{66}}{352}+\underset{\chi_{74}}{\mathbf{1 3} 28} \\
& \text { Top } U(4 / 35)^{c}=\underset{\chi_{5}}{1430}+\underset{\chi_{10}}{30030}+\underset{\chi_{67}}{2080}+\underset{\chi_{75}}{27456}
\end{aligned}
$$

where $\chi_{i}$ are the irreducible characters of $\mathrm{Fi}_{22}$ and $2 . \mathrm{Fi}_{22}$ labeled as in [ATLAS, pages $156-157]$.

By Lemma 5.5 and Proposition 5.7, the Griess algebra of the $C_{\mathrm{Fi}_{23}}\left(\tau_{f^{1}}\right)$-invariants of $\mathrm{Com}_{V^{\natural}}\langle a, b\rangle$ is 2-dimensional spanned by the $c=782 / 35$ conformal vector and the $c=25 / 28$ Virasoro vector $f^{1} \in \operatorname{Com}_{X^{[1]}} X^{[0]}$. Therefore, $f^{1}$ is the axial vector of the 2A-element $\tau_{f^{1}}$ of $\mathrm{Fi}_{23}$ and we have established the Conway-Miyamoto correspondence for $\mathrm{Fi}_{23}$.

Theorem 5.8. Let $E_{V^{\natural}}$ be the set of Ising vectors of $V^{\natural}$ and let $a, b \in E_{V^{\natural}}$ be a pair such that $\langle a, b\rangle \cong U_{3 A}$, i.e., $(a \mid b)=13 \cdot 2^{-10}$. Then there exists a Conway-Miyamoto correspondence between 2A-elements of $\mathrm{Fi}_{23}$ and $c=25 / 28$ Virasoro vectors of $\mathrm{Com}_{V^{\natural}}\langle a, b\rangle$. More precisely, set $I_{a, b}=\left\{x \in E_{V^{\natural}} \mid\langle a, x\rangle \cong\langle b, x\rangle \cong U_{2 A}\right\}$, i.e., $(a \mid x)=(b \mid x)=2^{-5}$ for any $x \in I_{a, b}$. Then the following hold.

(1) $C_{\mathbb{M}}\left(\tau_{a}, \tau_{b}\right)=\left\langle\tau_{x} \mid x \in I_{a, b}\right\rangle \cong \mathrm{Fi}_{23}$ acts faithfully on $\operatorname{Com}_{V^{\natural}}\langle a, b\rangle$.

(2) There is a one to one correspondence between $I_{a, b}$ and $2 A$-involutions of $\mathrm{Fi}_{23}$ via the Miyamoto involution $\left.x \longmapsto \tau_{x}\right|_{\mathrm{Com}_{V^{\natural}}\langle a, b\rangle}$.

(3) Given a 2A-involution $t$ of $\mathrm{Fi}_{23}$, there exists a unique $C_{\mathrm{Fi}_{23}}(t)$-invariant $c=25 / 28$ Virasoro vector $f$ of $\mathrm{Com}_{V \natural}\langle a, b\rangle$ such that $\tau_{f}=t$. Moreover, $\tau_{f}$ on $V^{\natural}$ defines a $2 A$ involution of $\mathbb{M}$.

For the inductive argument from $G^{[0]}=\mathrm{Fi}_{23}$ to $G^{[1]}=\mathrm{Fi}_{22}$, we determine the decomposition of the Griess algebra of $\mathrm{Com}_{V^{\natural}} X^{[1]}$ as a $\mathrm{Fi}_{22}$-module. 
Lemma 5.9. Consider the subalgebras $X^{[0]}=\langle a, b\rangle \subset X^{[1]}=\left\langle a, b, x^{1}\right\rangle \subset V^{\natural}$ as above and let $f^{1}$ be the conformal vector of $\mathrm{Com}_{X^{[1]}} X^{[0]}$. Then the zero-mode $\mathrm{o}\left(f^{1}\right)$ acts on the Griess algebra of $V^{\natural}$ semisimply with possible eigenvalues

$$
0,2, \frac{9}{7}, \frac{3}{4}, \frac{5}{14}, \frac{1}{28}, \frac{3}{28}, \frac{15}{28}, \frac{5}{32}, \frac{3}{224}, \frac{15}{224}, \frac{99}{224}, \frac{143}{224} .
$$

More precisely, o $\left(f^{1}\right)$ acts semisimply on the Griess algebra of $\operatorname{Com}_{V}\langle a, b\rangle$ and the top levels Top $U(h)^{c}$ with possible eigenvalues as follows.

$$
\begin{array}{ll}
\left(\operatorname{Com}_{V^{\natural}}\langle a, b\rangle\right)_{2}: 0,3 / 4,5 / 32, & \operatorname{Top} U(5 / 7)^{c}: 9 / 7,1 / 28,15 / 224, \\
\operatorname{Top} U(2 / 5)^{c}: 0,5 / 32, & \operatorname{Top} U(1 / 7)^{c}: 5 / 14,3 / 28,143 / 224,3 / 224, \\
\operatorname{Top} U(19 / 35)^{c}: 5 / 14,3 / 28,3 / 224, & \operatorname{Top} U(4 / 35)^{c}: 15 / 28,1 / 28,99 / 224,15 / 224 .
\end{array}
$$

Proof: Since the axial vector $f^{1}$ is fixed by $C_{\mathrm{Fi}_{23}}\left(\tau_{f^{1}}\right)$ by Theorem 5.8, its zero-mode $\mathrm{o}\left(f^{1}\right)$ acts as a scalar on each $C_{\mathrm{Fi}_{23}}\left(\tau_{f^{1}}\right)$-irreducible component. By (5.1), we have the following decomposition of the Griess algebra of $V^{\natural}$.

$$
V_{2}^{\natural}=\langle a, b\rangle_{2} \oplus\left(\operatorname{Com}_{V^{\natural}}\langle a, b\rangle\right)_{2} \oplus \bigoplus_{\substack{U(h) \in \operatorname{Irr}\langle a, b\rangle \\ h>0}} \operatorname{Top} U(h) \otimes \operatorname{Top} U(h)^{c} .
$$

The 6 A-algebra $\left\langle a, b \circ x^{1}\right\rangle=\left\langle a, b, x^{1}\right\rangle$ contains a rational full subVOA $\left\langle a, b, f^{1}\right\rangle=\langle a, b\rangle \otimes\left\langle f^{1}\right\rangle$ by (8) of Theorem 2.13. As explained in the beginning of this section, all Ising vectors of $V^{\natural}$ are contained in the compact real form $V_{\mathbb{R}}^{\natural}$ of $V^{\natural}$ so that $V^{\natural}$ is semisimple as a $\left\langle a, b, x^{1}\right\rangle$-module. The uniqueness of a simple VOA structure of the 6A-algebra as well as the classification of irreducible modules over the 6A-algebra are established in [DJY19]. By (loc. cit.), an irreducible $U_{6 \mathrm{~A}}$-module is isomorphic to one of the following as a $U_{3 \mathrm{~A}} \otimes L(25 / 28,0)$-module.

$$
\begin{array}{lll}
{[0,0] \oplus[1 / 7,34 / 7] \oplus[5 / 7,9 / 7],} & {[0,5 / 32] \oplus[1 / 7,675 / 224] \oplus[5 / 7 \otimes[99 / 224],} \\
{[1 / 7,3 / 224] \oplus[0,165 / 32] \oplus[5 / 7,323 / 224],} & {[19 / 35,3 / 224] \oplus[2 / 5,165 / 32] \oplus[4 / 35,323 / 224],} \\
{[1 / 7,5 / 14] \oplus[0,15 / 2] \oplus[5 / 7,39 / 14],} & {[19 / 35,5 / 14] \oplus[2 / 5,15 / 2] \oplus[4 / 35,39 / 14],} \\
{[2 / 5,0] \oplus[19 / 35,34 / 7] \oplus[4 / 35,9 / 7],} & {[4 / 35,15 / 224] \oplus[2 / 5,57 / 32] \oplus[19 / 35,143 / 224],} \\
{[4 / 35,1 / 28] \oplus[2 / 5,3 / 4] \oplus[19 / 35,45 / 28],} & {[1 / 7,3 / 28] \oplus[0,13 / 4] \oplus[5 / 7,15 / 28],} \\
{[0,3 / 4] \oplus[5 / 7,1 / 28] \oplus[1 / 7,45 / 28],} & {[1 / 7,143 / 224] \oplus[5 / 7,15 / 224] \oplus[0,57 / 32],} \\
{[19 / 35,3 / 28] \oplus[4 / 35,15 / 28] \oplus[2 / 5,13 / 4],} & {[2 / 5,5 / 32] \oplus[4 / 35,99 / 224] \oplus[19 / 35,675 / 224],}
\end{array}
$$

where $[h, k]$ denotes $U(h) \otimes L(25 / 28, k)$. By considering the decomposition in (5.2) together with the possible shapes in (5.3), we obtain the list of possible eigenvalues of $o\left(f^{1}\right)$ as in the assertion. 
In order to determine the o $\left(f^{1}\right)$-spectrum of the Griess algebra of $V^{\natural}$, we need traces $\operatorname{tr}_{V_{2}^{\natural}} \mathrm{O}(f)^{i}$ for $1 \leq i \leq 5$, which can be computed by the Matsuo-Norton trace formulae in [Ma01].

Lemma 5.10 ([Ma01]). The traces of $\mathrm{o}\left(f^{1}\right)^{i}, 1 \leq i \leq 5$, on the Griess algebra are as follows.

$$
\begin{aligned}
& \operatorname{tr}_{V_{2}^{\natural}} \mathrm{O}\left(f^{1}\right)=\frac{410175}{28}, \quad \operatorname{tr}_{V_{2}^{\natural}} \mathrm{O}\left(f^{1}\right)^{2}=\frac{2411375}{784}, \quad \operatorname{tr}_{V_{2}^{\natural}} \mathrm{O}\left(f^{1}\right)^{3}=\frac{27230625}{21952}, \\
& \operatorname{tr}_{V_{2}^{\natural}} \mathrm{O}\left(f^{1}\right)^{4}=\frac{793401325}{1229312}, \quad \operatorname{tr}_{V_{2}^{\natural}} \mathrm{O}\left(f^{1}\right)^{5}=\frac{15221783625}{39337984} .
\end{aligned}
$$

By Lemma 5.10 and possible eigenvalues in Lemma 5.9, we can determine the o $\left(f^{1}\right)$ spectrum compatible with the decompositions in Proposition 5.7. The result is as follows.

Proposition 5.11. The zero-mode o $\left(f^{1}\right)$ acts on the decomposition in (5.2) as follows.

$$
\begin{aligned}
& \left(\mathrm{Com}_{V^{\natural}}\langle a, b\rangle\right)_{2}=1+1+429+3080+13650+13728
\end{aligned}
$$

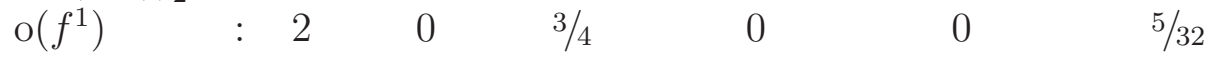

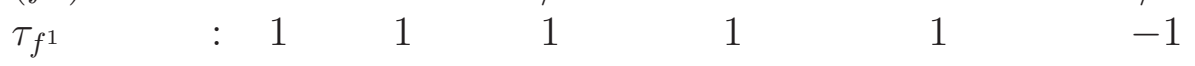

$$
\begin{aligned}
& \text { Top } U(5 / 7)^{c}=1+429+352 \\
& \mathrm{o}\left(f^{1}\right) \quad: \quad 9 / 7 \quad 1 / 28 \quad 15 / 224
\end{aligned}
$$

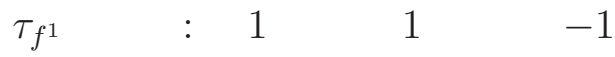

$$
\begin{aligned}
& \text { Top } U(19 / 35)^{c}=\mathbf{7 8}+\mathbf{1 4 3 0}+\mathbf{2 0 8 0} \\
& \mathrm{o}\left(f^{1}\right) \quad: \quad 5 / 14 \quad 3 / 28 \quad 3 / 224 \\
& \begin{array}{lllll}
\tau_{f^{1}} & : & 1 & 1 & -1
\end{array} \\
& \text { Top } U(2 / 5)^{c}=\mathbf{3 0 0 3}+\mathbf{2 0 8 0} \\
& \mathrm{o}\left(f^{1}\right) \quad: \quad 0 \quad 5 / 32 \\
& \begin{array}{llll}
\tau_{f^{1}} & : & 1 & -1
\end{array} \\
& \text { Top } U(1 / 7)^{c}=1001+10725+352+13728 \\
& \mathrm{o}\left(f^{1}\right) \quad: \quad 5 / 14 \quad 3 / 28 \quad 143 / 224 \quad 3 / 224 \\
& \begin{array}{llllll}
\tau_{f^{1}} & : & 1 & 1 & -1 & -1
\end{array} \\
& \text { Top } U(4 / 35)^{c}=1430+30030+2080+27456 \\
& \begin{array}{cccccc}
\mathrm{o}\left(f^{1}\right) & : & 15 / 28 & 1 / 28 & 99 / 224 & 15 / 224 \\
\tau_{f^{1}} & : & 1 & 1 & -1 & -1
\end{array}
\end{aligned}
$$

Proof: Since $\tau_{f^{1}}$ is the central element in $C_{\mathrm{Fi}_{23}}\left(\tau_{f^{1}}\right) \cong 2 . \mathrm{Fi}_{22}$, it acts as 1 on the irreducible $2 . \mathrm{Fi}_{22}$-components corresponding to the characters $\chi_{i}$ with $1 \leq i \leq 10$ and as -1 on those to $\chi_{i}$ with $i \geq 66$ (cf. [ATLAS]). If $\tau_{f^{1}}$ is trivial then the possible eigenvalues of $\mathrm{o}\left(f^{1}\right)$ are $0,2,9 / 7,3 / 4,5 / 14,1 / 28,3 / 28,15 / 28$ and if $\tau_{f^{1}}$ is non-trivial then the possible eigenvalues are $5 / 32,3 / 224,15 / 224,99 / 224,143 / 224$ by Lemma 5.9. This information 
together with Lemma 5.9 leads to the unique assignment of eigenvalues compatible with the set of traces in Lemma 5.10 and we obtain the eigenspace decompositions as in the assertion.

\subsection{Transpositions of $\mathrm{Fi}_{22}$ and $c=11 / 12$ Virasoro vectors}

Let $X^{[0]}=\langle a, b\rangle \subset X^{[1]}=\left\langle a, b, x^{1}\right\rangle \subset X^{[2]}=\left\langle a, b, x^{1}, x^{2}\right\rangle \subset V^{\natural}$ be the subalgebras of $V^{\natural}$ defined as in (4.13), and let $f^{1}$ and $f^{2}$ be the conformal vectors of $\operatorname{Com}_{X^{[1]}} X^{[0]}$ and $\operatorname{Com}_{X^{[2]}} X^{[1]}$, respectively. Let

$$
D^{[1]}=\left\{\tau_{y} \in D^{[0]} \mid \tau_{y} \tau_{x^{1}}=\tau_{x^{1}} \tau_{y}\right\}, \quad G^{[1]}=\left\langle\varphi^{[1]}\left(D^{[1]}\right)\right\rangle \subset \operatorname{Aut}\left(\operatorname{Com}_{V \natural}\left\langle a, b, x^{1}\right\rangle\right)
$$

be defined as in (4.19) and (4.20). We have shown in Proposition 5.4 that $G^{[1]} \cong \mathrm{Fi}_{22}$. In the following we will identify $\mathrm{Fi}_{22}$ with $G^{[1]}$. Since $\left\langle a, b, f^{1}\right\rangle \cong\langle a, b\rangle \otimes\left\langle f^{1}\right\rangle$ is a full subalgebra of $X^{[1]}=\left\langle a, b, x^{1}\right\rangle$, the commutant $\operatorname{Com}_{V^{\natural}}\left\langle a, b, x^{1}\right\rangle$ coincides with the 0-eigenspace of $\mathrm{o}\left(f^{1}\right)$ in $\operatorname{Com}_{V^{\natural}}\langle a, b\rangle$. Therefore, by Proposition 5.11, we have the following decomposition of the Griess algebra of $V F_{22}^{\natural}=\operatorname{Com}_{V^{\natural}}\left\langle a, b, x^{1}\right\rangle$ as a $\mathrm{Fi}_{22}$-module.

Proposition 5.12. As a $G^{[1]} \simeq \mathrm{Fi}_{22}$-module, the Griess algebra of $\operatorname{Com}_{V}\left\langle a, b, x^{1}\right\rangle$ decomposes as follows.

$$
\left(\mathrm{Com}_{V \natural}\left\langle a, b, x^{1}\right\rangle\right)_{2}=\begin{gathered}
\mathbf{1} \\
\chi_{1}
\end{gathered} \quad \begin{gathered}
\mathbf{3 0 8 0} \\
\chi_{7}
\end{gathered} \quad \begin{gathered}
\mathbf{1 3 6 5 0} \\
\chi_{9}
\end{gathered}
$$

where $\chi_{1}, \chi_{7}$ and $\chi_{9}$ are irreducible characters of $\mathrm{Fi}_{22}$ labeled as in [ATLAS, page 156].

It is shown in Proposition 5.4 that $\varphi^{[1]}\left(\tau_{x^{2}}\right)$ defines a $2 \mathrm{~A}$-element of $\mathrm{Fi}_{22}$ and its centralizer is $C_{\mathrm{Fi}_{22}}\left(\varphi^{[1]}\left(\tau_{x^{2}}\right)\right) \cong 2 . \mathrm{PSU}_{6}(2)$. We have the following decompositions.

Lemma 5.13. As a $C_{\mathrm{Fi}_{22}}\left(\varphi^{[1]}\left(\tau_{x^{2}}\right)\right) \cong 2 . \mathrm{PSU}_{6}(2)$-module, we have

$$
\begin{aligned}
& \begin{array}{c}
3080 \\
\chi_{7}
\end{array}=\begin{array}{c}
1 \\
\mu_{1}
\end{array}+\underset{\mu_{4}}{252}+\underset{\mu_{6}}{440}+\begin{array}{c}
1155 \\
\mu_{11}
\end{array} \quad \begin{array}{c}
\mathbf{1 2 3 2} \\
\mu_{51}
\end{array} \\
& \begin{array}{c}
13650 \\
\chi_{9}
\end{array}=\underset{\mu_{4}}{252}+\underset{\mu_{12}}{1155}+\underset{\mu_{13}}{1155}+\underset{\mu_{20}}{4928}+\underset{\mu_{57}}{\mathbf{6 1 6 0}}
\end{aligned}
$$

where $\chi_{i}$ are the irreducible characters of $\mathrm{Fi}_{22}$ as in the previous proposition, and $\mu_{i}$ are the irreducible characters of $\mathrm{PSU}_{6}(2)$ and $2 . \mathrm{PSU}_{6}(2)$ labeled as in [ATLAS, page 116].

It follows from Lemma 5.5, Proposition 5.12 and Lemma 5.13 that the Griess algebra of the $C_{\mathrm{Fi}_{22}}\left(\varphi^{[1]}\left(\tau_{x^{2}}\right)\right)$-invariants of $\operatorname{Com}_{V^{\natural}}\left\langle a, b, x^{1}\right\rangle$ is 2-dimensional spanned by the $c=429 / 20$ conformal vector and the $c=11 / 12$ Virasoro vector $f^{2}$ in $\operatorname{Com}_{X^{[2]}} X^{[1]}$. It also follows from (2) of Theorem 4.19 that $f^{2}$ is of $\sigma$-type and satisfies $\sigma_{f^{2}}=\varphi^{[1]}\left(\tau_{x^{2}}\right)$ on $\mathrm{Com}_{V^{\natural}}\left\langle a, b, x^{1}\right\rangle$. Therefore, we have obtained the Conway-Miyamoto correspondence between 2A-involutions of $\mathrm{Fi}_{22}$ and $c=11 / 12$ Virasoro vectors of $\sigma$-type of $\operatorname{Com}_{V^{\natural}}\left\langle a, b, x^{1}\right\rangle$. 
Theorem 5.14. Let $E_{V^{\natural}}$ be the set of Ising vectors of $V^{\natural}$ and let $a, b \in E_{V^{\natural}}$ be a pair such that $\langle a, b\rangle \cong U_{3 A}$, i.e., $(a \mid b)=13 \cdot 2^{-10}$. Set $I_{a, b}=\left\{x \in E_{V^{\natural}} \mid\langle a, x\rangle \cong\langle b, x\rangle \cong U_{2 A}\right\}$, i.e., $(a \mid x)=(b \mid x)=2^{-5}$ for any $x \in I_{a, b}$, and take $x^{1} \in I_{a, b}$. Then there exists $a$ Conway-Miyamoto correspondence between $2 A$-elements of $\mathrm{Fi}_{22}$ and $c=11 / 12$ Virasoro vectors of $\sigma$-type of $\mathrm{Com}_{V^{\natural}}\left\langle a, b, x^{1}\right\rangle$. More precisely, the following hold.

(1) Set $D^{[1]}=\left\{\tau_{y} \mid y \in I_{a, b}, \tau_{x^{1}} \tau_{y}=\tau_{y} \tau_{x^{1}}\right\}$. Then $\left\langle D^{[1]}\right\rangle=C_{\mathbb{M}}\left(\tau_{a}, \tau_{b}, \tau_{x^{1}}\right) \cong 2$.Fi T2 $_{22}$ acts on $\mathrm{Com}_{V^{\natural}}\left\langle a, b, x^{1}\right\rangle$ with the kernel $\left\langle\tau_{x^{1}}\right\rangle$.

(2) Let $\varphi^{[1]}:\left\langle D^{[1]}\right\rangle \rightarrow \operatorname{Aut}\left(\mathrm{Com}_{V^{\natural}}\left\langle a, b, x^{1}\right\rangle\right)$ be the homomorphism given in (1) and $G^{[1]}$ the image of $\varphi^{[1]}$. Then $G^{[1]} \cong \mathrm{Fi}_{22}$ and $\left.\varphi^{[1]}\left(D^{[1]}\right)\right)$ is the set of $2 A$-involutions of $\mathrm{Fi}_{22}$.

(3) Given a 2A-involution $t$ of $\mathrm{Fi}_{22}$, there exists a unique $C_{\mathrm{Fi}_{22}}(t)$-invariant $c=11 / 12$ Virasoro vector $f$ of $\sigma$-type of $\operatorname{Com}_{V \sharp}\left\langle a, b, x^{1}\right\rangle$ such that $\sigma_{f}=t$.

Remark 5.15. In principle, we can continue to consider the next case $X^{[2]} \subset X^{[3]}=$ $\left\langle a, b, x^{1}, x^{2}, x^{3}\right\rangle$ and study $\operatorname{Com}_{V^{\natural}} X^{[2]}$ as a $G^{[2]} \cong \operatorname{PSU}_{6}(2)$-module. Let $f^{3}$ be the $c=$ $c_{7}=14 / 15$ Virasoro vector of $\operatorname{Com}_{X^{[3]}} X^{[2]}$. We know that $\tau_{f^{3}}=\varphi^{[2]}\left(\tau_{x^{3}}\right)$ defines a 2Aelement of $\mathrm{PSU}_{2}(6)$ on $\mathrm{Com}_{V^{\natural}} X^{[2]}$ by (1) of Theorem 4.19 and Proposition 5.4. In order to verify the Conway-Miyamoto correspondence for $\mathrm{Fi}_{21}=\operatorname{PSU}_{6}(2)$, we have to compute the $C_{\mathrm{Fi}_{21}}\left(\tau_{f^{3}}\right)$-invariants of the Griess algebra of $\operatorname{Com}_{V^{\natural}} X^{[2]}$. However, the Griess algebra of $\mathrm{Com}_{V^{\natural}} X^{[1]}$ splits into many pieces as in Lemma 5.13 and its 0-eigenspace of o $\left(f^{2}\right)$ is technically difficult to determine so that we cannot obtain the decomposition of the Griess algebra of $\mathrm{Com}_{V^{\natural}} X^{[2]}$ at this moment. It is likely that the $C_{\mathrm{Fi}_{21}}\left(\tau_{f^{3}}\right)$-invariants of the Griess algebra of of $\operatorname{Com}_{V^{\natural}} X^{[2]}$ has a dimension more than 2, and the ConwayMiyamoto correspondence for $\mathrm{Fi}_{21}$ seems to fail. However, even the $C_{\mathrm{Fi}_{21}}\left(\tau_{f}\right)$-invariants is larger, we still have a chance that it contains a unique $c=c_{7}$ Virasoro vector.

\section{Appendix A Explicit constructions}

We will give explicit constructions of the subalgebras discussed in Section 4.

\section{A.1 Compact real form of a lattice VOA}

Let $L$ be an even positive definite lattice. Let $V_{L}$ be the associated lattice VOA defined over $\mathbb{C}$ and $V_{L, \mathbb{R}}$ the one defined over $\mathbb{R}$ (cf. [FLM88]). Let $\theta$ be a lift of the (-1)-isometry of $L$ on $V_{L, \mathbb{R}}$ and $V_{L, \mathbb{R}}=V_{L, \mathbb{R}}^{+} \oplus V_{L, \mathbb{R}}^{-}$the eigenspace decomposition such that $\theta$ acts by \pm 1 on $V_{L, \mathbb{R}}^{ \pm}$. Then the invariant bilinear form on $V_{L, \mathbb{R}}$ is positive definite on $V_{L, \mathbb{R}}^{+}$and negative definite on $V_{L, \mathbb{R}}^{-}$. Therefore, the real subspace

$$
\left(V_{L}\right)_{\mathbb{R}}:=V_{L, \mathbb{R}}^{+} \oplus \sqrt{-1} V_{L, \mathbb{R}}^{-}
$$


forms a compact real subalgebra of $V_{L}$. In the following We will use the compact form $\left(V_{L}\right)_{\mathbb{R}}$ in $(\mathrm{A} .1)$ of $V_{L}$.

Remark A.1. In the construction of a lattice vertex operator algebra $V_{L}$ one needs to implement a 2-cocycle $\varepsilon \in Z^{2}(L,\{ \pm 1\})$ such that $\varepsilon(a, b) \varepsilon(b, a)=(-1)^{(a \mid b)}$ for $a, b \in L$. If $L$ is of rank one or doubly even, then we can take $\varepsilon$ to be trivial.

\section{A.2 Virasoro vectors associated to root systems}

We recall the construction of certain Virasoro vectors from [DLMN98]. Let $R$ be a root lattice and $\Phi(R)$ its root system. Fix a system of simple roots and let $\Phi^{+}(R)$ and $\Phi^{-}(R)$ be the set of positive and negative roots, respectively. We use $\sqrt{2} R$ to denote the $\mathbb{Z}$ -

submodule $\sqrt{2} \otimes_{\mathbb{Z}} R$ of $\mathbb{R} \otimes_{\mathbb{Z}} R$ which forms a doubly even lattice. By [DLMN98], the conformal vector $\omega_{R}$ of the lattice $\operatorname{VOA} V_{\sqrt{2} R}$ is given by

$$
\omega_{R}=\frac{1}{4 h} \sum_{\alpha \in \Phi(R)} \alpha_{(-1)}^{2} \mathbb{1}
$$

where $h$ is the Coxeter number of $R$. For $\alpha \in \sqrt{2} R$ with $(\alpha \mid \alpha)=4$ we set

$$
w^{ \pm}(\alpha):=\frac{1}{16} \alpha_{(-1)}^{2} \mathbb{1} \pm \frac{1}{4}\left(e^{\alpha}+e^{-\alpha}\right) .
$$

Then $w^{ \pm}(\alpha)$ are Ising vectors (cf. [DMZ94]). The following is straight forward.

Lemma A.2. Let $\alpha, \beta \in \sqrt{2} R$ be squared norm 4 vectors. Then

$$
\left(w^{\varepsilon}(\alpha) \mid w^{\varepsilon^{\prime}}(\beta)\right)= \begin{cases}2^{-2} & \text { if } \alpha= \pm \beta \text { and } \varepsilon=\varepsilon^{\prime}, \\ 0 & \text { if }(\alpha \mid \beta)=0 \text { or } \alpha= \pm \beta \text { with } \varepsilon=-\varepsilon^{\prime}, \\ 2^{-5} & \text { if }(\alpha \mid \beta)= \pm 2,\end{cases}
$$

where $w^{ \pm}(\alpha)$ and $w^{ \pm}(\beta)$ are defined as in (A.2). Moreover, we have

$$
w^{\varepsilon}(\alpha)_{(1)} w^{\epsilon^{\prime}}(\beta)=\frac{1}{4}\left(w^{\varepsilon}(\alpha)+w^{\varepsilon^{\prime}}(\beta)-w^{-\varepsilon \varepsilon^{\prime}}(\alpha-\beta)\right)
$$

if $(\alpha \mid \beta)= \pm 2$.

We also define

$$
\begin{aligned}
s_{R} & :=\frac{4}{(h+2)} \sum_{\alpha \in \sqrt{2} \Phi^{+}(R)} w^{-}(\alpha), \\
t_{R} & :=\omega_{R}-s_{R}=\frac{2}{h+2} \omega_{R}+\frac{1}{h+2} \sum_{\alpha \in \sqrt{2} \Phi(R)} e^{\alpha} .
\end{aligned}
$$


Then it is shown in [DLMN98] that $s_{R}$ and $t_{R}$ are mutually orthogonal Virasoro vectors. The central charge of $t_{R}$ is $2 n /(n+3)$ if $R$ is of type $A_{n}, 1$ if $R$ is of type $D_{n}$, and 6/7,7/10 and $1 / 2$ if $R$ is of type $E_{6}, E_{7}$ and $E_{8}$, respectively. By Lemma A.2, the linear span of $w^{-}(\alpha)$ with $\alpha \in \sqrt{2} \Phi(R)$ forms a subalgebra of the Griess algebra of $V_{\sqrt{2}}^{+}$. Indeed, this is the Griess algebra of the subVOA generated by the Ising vectors $w^{-}(\alpha), \alpha \in \sqrt{2} \Phi(R)$. The Virasoro vector $s_{R}$ provides its conformal vector and by the orthogonality we have the following (cf. Proposition 5.1 and Lemma 5.7 of [LSY07]).

Proposition A.3 ([LSY07]). $\left\langle w^{-}(\alpha) \mid \alpha \in \sqrt{2} \Phi(R)\right\rangle=\operatorname{Com}_{V_{\sqrt{2} R}^{+}}\left\langle t_{R}\right\rangle$.

The subVOA $\left\langle w^{-}(\alpha) \mid \alpha \in \sqrt{2} \Phi(R)\right\rangle$ is denoted by $M_{R}$ and its automorphism group is determined in [LSY07].

Remark A.4. If $R=E_{8}$, then

$$
t_{E_{8}}=\frac{1}{16} \omega_{E_{8}}+\frac{1}{32} \sum_{\alpha \in \sqrt{2} \Phi\left(E_{8}\right)} e^{\alpha}
$$

is an Ising vector. We say that $t_{E_{8}}$ is the standard Ising vector of $V_{\sqrt{2} E_{8}}^{+}$. Recall that the dual lattice $\left(\sqrt{2} E_{8}\right)^{*}=\frac{1}{\sqrt{2}} E_{8}$. For $x \in\left(\sqrt{2} E_{8}\right)^{*}$, define a $\mathbb{Z}$-linear map

$$
\begin{aligned}
(x \mid \cdot): \sqrt{2} E_{8} & \longrightarrow(x \mid y) \quad \mathbb{Z}_{2} \\
y & \longmapsto(\bmod 2 .
\end{aligned}
$$

Clearly the map

$$
\begin{array}{cccc}
\varphi:\left(\sqrt{2} E_{8}\right)^{*} & \longrightarrow & \operatorname{Hom}_{\mathbb{Z}}\left(\sqrt{2} E_{8}, \mathbb{Z}_{2}\right) \\
x & \longmapsto & (x \mid \cdot)
\end{array}
$$

is a group homomorphism and $\operatorname{Ker} \varphi=\sqrt{2} E_{8}$. The map $(x \mid \cdot)$ thus induces an automorphism $\varphi_{x}$ of $V_{\sqrt{2} E_{8}}$ given by

$$
\varphi_{x}\left(u \otimes e^{\alpha}\right)=(-1)^{(x \mid \alpha)} u \otimes e^{\alpha} \quad \text { for } u \in M(1) \text { and } \alpha \in \sqrt{2} E_{8} .
$$

In this case,

$$
\varphi_{x} t_{E_{8}}=\frac{1}{16} \omega_{E_{8}}+\frac{1}{32} \sum_{\alpha \in \sqrt{2} \Phi\left(E_{8}\right)}(-1)^{(x \mid \alpha)} e^{\alpha}
$$

is also an Ising vector. Since $\varphi_{x}$ commutes $\theta$ (the lift of the -1 isometry), the Ising vector $\varphi_{x} t_{E_{8}}$ is contained in $V_{\sqrt{2} E_{8}}^{+}$. The Ising vectors in $V_{\sqrt{2} E_{8}}^{+}$have been classified in [G98a, LSY07, LS07]. There are $240+256=496$ Ising vectors and they can be divided into two different types:

$A_{1}$-type: $w^{ \pm}(\alpha), \alpha \in \sqrt{2} E_{8},(\alpha \mid \alpha)=4 \quad\left(\left|\Phi\left(E_{8}\right)\right|=240\right.$ Ising vectors $)$,

$E_{8}$-type: $\varphi_{x} t_{E_{8}}$ for some $x \in \frac{1}{\sqrt{2}} E_{8} \quad\left(2^{8}=256\right.$ Ising vectors $)$. 
Remark A.5. Let $\alpha_{1}, \ldots, \alpha_{n}$ be the simple roots of a root lattice of type $A_{n}$, that is, $\left(\alpha_{i} \mid \alpha_{i}\right)=2,\left(\alpha_{i} \mid \alpha_{j}\right)=-1$ if $|i-j|=1$ and $\left(\alpha_{i} \mid \alpha_{j}\right)=0$ otherwise. Then

$$
\operatorname{Span}_{\mathbb{Z}}\left\{\alpha_{1}, \ldots, \alpha_{k}\right\} \cong A_{k}
$$

for any $1 \leq k \leq n$ and we have a sequence of sublattices

$$
A_{1} \subset A_{2} \subset \cdots \subset A_{n}
$$

For $1 \leq k \leq n$, denote $s^{k}=s_{A_{k}}$. We also set

$$
\eta^{1}=s^{1} \quad \text { and } \quad \eta^{k}=s^{k}-s^{k-1}
$$

for $2 \leq k \leq n$. It is shown in [DLMN98] (see also [GKO86]) that $\eta^{k}$ is a simple Virasoro vector of central charge

$$
c_{k}=1-\frac{6}{(k+2)(k+3)}, \quad 1 \leq k \leq n .
$$

Moreover, we have the following orthogonal decomposition of the conformal vector of $V_{\sqrt{2}} A_{n}$.

$$
\omega_{A_{n}}=\eta^{1} \dot{+} \eta^{2} \dot{+} \cdots \dot{+} \eta^{n} \dot{+} t_{A_{n}} .
$$

\section{A.3 Construction of $X^{[n]}$}

Let $n \geq 1$ and $\epsilon_{1}, \ldots \epsilon_{n+5}$ an orthogonal basis of $\mathbb{R}^{n}$ such that $\left(\epsilon_{i} \mid \epsilon_{j}\right)=2 \delta_{i, j}$. Set

$$
K^{[k]}=\mathbb{Z} \epsilon_{1} \oplus \mathbb{Z} \epsilon_{2} \oplus \cdots \oplus \mathbb{Z} \epsilon_{k}, \quad 1 \leq k \leq n+5 .
$$

Then $K^{[k]} \cong A_{1}^{\oplus k}$. Set $\gamma=\frac{1}{2}\left(\epsilon_{1}+\epsilon_{2}+\epsilon_{3}+\epsilon_{4}\right)$ and $L=K^{[n+5]} \sqcup\left(K^{[n+5]}+\gamma\right)$. Then $L \cong D_{4} \oplus A_{1}^{\oplus(n+5)}$. We consider lattice VOAs

$$
V_{K^{[1]}} \subset V_{K^{[2]}} \subset \cdots \subset V_{K^{[n+5]}} \subset V_{L}
$$

Note that $V_{K^{[n+5]}}$ is the full subVOA of $V_{L}$. We denote the conformal vector of $V_{L}$ by $\omega_{L}$.

Remark A.6. Since $(\alpha \mid \beta) \in 2 \mathbb{Z}$ for any $\alpha, \beta \in K^{[n+5]}$, we can define $V_{K^{[n+5]}}$ with a trivial 2-cocycle in $Z^{2}\left(K^{[n+5]},\{ \pm 1\}\right)$. In the construction of $V_{L}$, we choose a 2-cocycle in $Z^{2}(L,\{ \pm 1\})$ such that its restriction on $K^{[n+5]}$ is trivial. This is possible since we can form $V_{L}=V_{K^{[n+5]}} \oplus V_{K^{[n+5]}+\gamma}$ as a $\mathbb{Z}_{2^{\text {-graded }}}$ simple current extension of $V_{K^{[n+5]}}$.

For $1 \leq k \leq n+5$, we set

$$
\begin{aligned}
& H^{k}=\epsilon_{1(-1)^{2}} \mathbb{1}+\epsilon_{2(-1)}^{2} \mathbb{1}+\cdots+\epsilon_{k(-1)}^{2} \mathbb{1}, \\
& E^{k}=e^{\epsilon_{1}}+e^{\epsilon_{2}}+\cdots+e^{\epsilon_{k}}, \\
& F^{k}=e^{-\epsilon_{1}}+e^{-\epsilon_{2}}+\cdots+e^{-\epsilon_{k}} .
\end{aligned}
$$


Then $H^{k}, E^{k}, F^{k}$ form an $\mathfrak{s l}_{2}$-triple in the weight one subspace of $V_{K^{[k]}}$ and generate a subVOA isomorphic to level $k$ affine $\operatorname{VOA} L_{\hat{\mathfrak{s l}}_{2}}(k, 0)$ associated to $\hat{\mathfrak{s l}}_{2}$. Let

$$
\Omega^{k}=\frac{1}{4(k+2)}\left(H_{(-1)}^{k} H^{k}+2 E_{(-1)}^{k} F^{k}+2 F_{(-1)}^{k} E^{k}\right)
$$

be the Sugawara element. Then $\Omega^{k}$ is the conformal vector of $L_{\hat{\mathfrak{s l}}_{2}}(k, 0)$ (cf. [FZ92]). Consider the sublattice

$$
M^{[k]}=\left\{\alpha \in K^{[k+1]} \mid\left(\alpha \mid \epsilon_{1}+\cdots+\epsilon_{n+5}\right)=0\right\},
$$

Then $M^{[k]} \cong \sqrt{2} A_{k}$ and we can define the Virasoro vectors

$$
s^{k}=\frac{4}{k+3} \sum_{1 \leq i<j \leq k+1} w^{-}\left(\epsilon_{i}-\epsilon_{j}\right), \quad \eta^{1}=w^{-}\left(\epsilon_{1}-\epsilon_{2}\right), \quad \eta^{k}=s^{k}-s^{k-1}
$$

for $2 \leq k \leq n+4$ as in (A.3) and (A.6). It is shown in [GKO86, LY04] that the Virasoro vectors $\eta^{k}$ can be written as

$$
\eta^{k}=\Omega^{k}+\frac{1}{4} \epsilon_{k+1(-1)}^{2} \mathbb{1}-\Omega^{k+1} .
$$

Therefore, we have the following orthogonal decompositions.

$$
\omega_{L}=s^{n+4} \dot{+} \Omega^{n+5}=\eta^{1} \dot{+} \eta^{2} \dot{+} \cdots \dot{+} \eta^{n+4} \dot{+} \Omega^{n+5} .
$$

As a consequence, we have the following full subVOA of $V_{L}$.

$$
L\left(c_{1}, 0\right) \otimes L\left(c_{2}, 0\right) \otimes \cdots \otimes L\left(c_{n+4}, 0\right) \otimes L_{\hat{\mathfrak{s l}}_{2}}(n+5,0) .
$$

We note that all $\eta^{k}, 1 \leq k \leq n+4$, and $\Omega^{n+5}$ are defined inside the compact form $V_{L, \mathbb{R}}^{+}$.

Lemma A.7. The weight one subspace of $\mathrm{Com}_{V_{L}}\left\langle\eta^{1}, \eta^{2}, \Omega^{n+5}\right\rangle$ is trivial.

Proof: It suffices to show $\operatorname{Ker}_{V_{L}} \eta_{(1)}^{1} \cap \operatorname{Ker}_{V_{L}} \eta_{(1)}^{2} \cap \operatorname{Ker}_{V_{L}} \Omega^{n+5}{ }_{(1)} \cap\left(V_{L}\right)_{1}=0$. Since $V_{L}=V_{K^{[n+5]}} \oplus V_{K^{[n+5]}+\gamma}$, we have a decomposition $\left(V_{L}\right)_{1}=\left(V_{K^{[n+5]}}\right)_{1} \oplus\left(V_{K^{[n+5]}+\gamma}\right)_{1}$. The weight one subspace of $V_{K^{[n+5]}}$ is $3(n+5)$-dimensional spanned by $\epsilon_{i(-1)} \mathbb{1}$ and $e^{ \pm \epsilon_{i}}$ for $1 \leq i \leq n+5$. By a direct computation one has

$$
\begin{aligned}
& \Omega_{(1)}^{n+5} \epsilon_{i(-1)} \mathbb{1}=\frac{2}{n+7} \epsilon_{i(-1)} \mathbb{1}+\frac{1}{n+7} H^{n+5}, \\
& \Omega_{(1)}^{n+5} e^{\epsilon_{i}}=\frac{2}{n+7} e^{\epsilon_{i}}+\frac{1}{n+7} E^{n+5}, \\
& \Omega^{n+5}{ }_{(1)} e^{-\epsilon_{i}}=\frac{2}{n+7} e^{-\epsilon_{i}}+\frac{1}{n+7} F^{n+5} .
\end{aligned}
$$


Therefore, the characteristic polynomial of $\Omega^{n+5}(1)$ on $\left(V_{K^{[n+5]}}\right)_{1}$ is

$$
(x-1)^{3}\left(x-\frac{2}{n+7}\right)^{3 n+12}
$$

and hence $\operatorname{Ker}_{V_{L}} \Omega^{n+5}{ }_{(1)} \cap\left(V_{K^{[n+5]}}\right)_{1}=0$. The weight one subspace of $V_{K^{[n+5]}+\gamma}$ is spanned by the vectors $e^{\alpha}$ with $\alpha=\frac{1}{2}\left( \pm \epsilon_{1} \pm \epsilon_{2} \pm \epsilon_{3} \pm \epsilon_{4}\right)$. Set $\gamma_{1}=\frac{1}{2}\left(\epsilon_{1}+\epsilon_{2}-\epsilon_{3}-\epsilon_{4}\right), \gamma_{2}=$ $\frac{1}{2}\left(\epsilon_{1}-\epsilon_{2}-\epsilon_{3}+\epsilon_{4}\right), \gamma_{3}=\frac{1}{2}\left(\epsilon_{1}-\epsilon_{2}+\epsilon_{3}-\epsilon_{4}\right)$ and $\tilde{e}\left(\gamma_{i}\right)=e^{\gamma_{i}}+e^{-\gamma_{i}}$ for $1 \leq i \leq 3$. Then by a direct computation one has

$$
\begin{aligned}
& \operatorname{Ker}_{V_{L}} \Omega_{(1)}^{n+5} \cap\left(V_{K^{[n+5]}+\gamma}\right)_{1}=\operatorname{Span}\left\{\tilde{e}\left(\gamma_{1}\right)-\tilde{e}\left(\gamma_{2}\right), \tilde{e}\left(\gamma_{2}\right)-\tilde{e}\left(\gamma_{3}\right)\right\}, \\
& \operatorname{Ker}_{V_{L}} \eta_{(1)}^{1} \cap \operatorname{Ker}_{V_{L}} \Omega^{n+5}{ }_{(1)} \cap\left(V_{K^{[n+5]}+\gamma}\right)_{1}=\operatorname{Span}\left\{2 \tilde{e}\left(\gamma_{1}\right)-\tilde{e}\left(\gamma_{2}\right)-\tilde{e}\left(\gamma_{3}\right)\right\}, \\
& \eta_{(1)}^{2}\left(2 \tilde{e}\left(\gamma_{1}\right)-\tilde{e}\left(\gamma_{2}\right)-\tilde{e}\left(\gamma_{3}\right)\right)=\frac{3}{5}\left(2 \tilde{e}\left(\gamma_{1}\right)-\tilde{e}\left(\gamma_{2}\right)-\tilde{e}\left(\gamma_{3}\right)\right) .
\end{aligned}
$$

Therefore, $\operatorname{Ker}_{V_{L}} \eta^{1}{ }_{(1)} \cap \operatorname{Ker}_{V_{L}} \eta^{2}{ }_{(1)} \cap \operatorname{Ker}_{V_{L}} \Omega^{n+5}{ }_{(1)} \cap\left(V_{K^{[n+5]}+\gamma}\right)_{1}=0$ and thus the weight one subspace of $\operatorname{Com}_{V_{L}}\left\langle\eta^{1}, \eta^{2}, \Omega^{n+5}\right\rangle$ is trivial.

Corollary A.8. The subalgebra $\operatorname{Com}_{V_{L, \mathbb{R}}^{+}}\left\langle\eta^{1}, \eta^{2}, \Omega^{n+5}\right\rangle$ is a compact VOA of OZ-type.

We will realize the $(2 \mathrm{~A}, 3 \mathrm{~A})$-generated subalgebra $X^{[n]}=\left\langle a, b, x^{1}, \ldots, x^{n}\right\rangle$ in Section 4.3 as a subalgebra of $\operatorname{Com}_{V_{L, \mathbb{R}}^{+}}\left\langle\eta^{1}, \eta^{2}, \Omega^{n+5}\right\rangle$. Set

$$
\begin{aligned}
q:= & \sum_{k=1}^{3}\left(2 \gamma+\epsilon_{k}-\epsilon_{4}-4 \epsilon_{5}\right)_{(-1)}\left(e^{\gamma-\epsilon_{k}-\epsilon_{4}}-e^{-\gamma+\epsilon_{k}+\epsilon_{4}}\right) \\
& -4 \sum_{k=1}^{3}\left(e^{\gamma-\epsilon_{k}-\epsilon_{5}}+e^{-\gamma+\epsilon_{k}+\epsilon_{5}}\right)+12\left(e^{\gamma-\epsilon_{4}-\epsilon_{5}}+e^{-\gamma+\epsilon_{4}+\epsilon_{5}}\right) \in V_{L, \mathbb{R}}^{+} .
\end{aligned}
$$

The following is shown in [SY03].

Lemma A.9 ([SY03]). The vector $q$ is a highest weight vectors for $\eta^{1}, \eta^{2}, \eta^{3}, \eta^{4}, \Omega^{n+5}$ and $w^{-}\left(\epsilon_{4}-\epsilon_{5}\right)$ with highest weights $0,0,2 / 3,4 / 3,0$ and $1 / 16$, respectively. In particular, $q \in \mathrm{Com}_{V_{L, \mathbb{R}}^{+}}\left\langle\eta^{1}, \eta^{2}, \Omega^{n+5}\right\rangle$.

We set

$$
a:=w^{-}\left(\epsilon_{4}-\epsilon_{5}\right), \quad b:=-\frac{1}{2} a+\frac{15}{64} \eta^{3}+\frac{21}{32} \eta^{4}+\frac{\sqrt{3}}{2^{7}} q, \quad c:=\tau_{a} b .
$$

Then it is shown in [SY03] that $a$ and $b$ are Ising vectors in $V_{L}$ such that $\langle a, b\rangle$ is isomorphic to the 3A-algebra with the characteristic Virasoro frame $\eta^{3} \dot{+} \eta^{4}$. For $1 \leq i \leq n$, we set

$$
x^{i}:=w^{-}\left(\epsilon_{5}-\epsilon_{i+5}\right)
$$

Using Lemma A.2 we can verify the following. 
Lemma A.10. $\left(a \mid x^{i}\right)=\left(b \mid x^{i}\right)=\left(x^{j} \mid x^{k}\right)=2^{-5}$ for $1 \leq i \leq n$ and $1 \leq j<k \leq n$.

Lemma A.11. $\left\langle a, b, x^{1}, \ldots, x^{n}\right\rangle$ is a subalgebra of $\operatorname{Com}_{V_{L, \mathbb{R}}^{+}}\left\langle\eta^{1}, \eta^{2}, \Omega^{n+5}\right\rangle$.

Proof: It follows from Proposition A.3 and Eq. (A.12) that $a=w^{-}\left(\epsilon_{4}-\epsilon_{5}\right)$ and $x^{i}=$ $w^{-}\left(\epsilon_{5}-\epsilon_{i+5}\right), 1 \leq i \leq n$, belong to the commutant of $\left\langle\Omega^{n+5}\right\rangle$. It also follows from Proposition A.3 that $s^{2}=\eta^{1} \dot{+} \eta^{2}$ is the conformal vector of $\left\langle w^{-}\left(\epsilon_{1}-\epsilon_{2}\right), w^{-}\left(\epsilon_{2}-\epsilon_{3}\right)\right\rangle$. By Lemma A.2, $a$ and $x^{i}, 1 \leq i \leq n$, are orthogonal to both of $w^{-}\left(\epsilon_{1}-\epsilon_{2}\right)$ and $w^{-}\left(\epsilon_{2}-\epsilon_{3}\right)$. Therefore $\left\langle a, x^{1}, \ldots, x^{n}\right\rangle \subset \operatorname{Com}_{V_{L, \mathbb{R}}^{+}}\left\langle\eta^{1}, \eta^{2}, \Omega^{n+5}\right\rangle$. That $b \in \operatorname{Com}_{V_{L, \mathbb{R}}^{+}}\left\langle\eta^{1}, \eta^{2}, \Omega^{n+5}\right\rangle$ follows from Lemma A.9. Hence, $\left\langle a, b, x^{1}, \ldots, x^{n}\right\rangle$ is a subalgebra of $\operatorname{Com}_{V_{L, \mathbb{R}}^{+}}\left\langle\eta^{1}, \eta^{2}, \Omega^{n+5}\right\rangle$.

Remark A.12. The Ising vectors in (A.15) and (A.16) are defined inside $V_{L, \mathbb{R}}^{+}$so that $\left\langle a, b, x^{1}, \ldots, x^{n}\right\rangle$ has a compact real form as a subalgebra of $\operatorname{Com}_{V_{L, \mathbb{R}}^{+}}\left\langle\eta^{1}, \eta^{2}, \Omega^{n+5}\right\rangle$ which is of OZ-type by Corollary A.8. Since $\langle a, b\rangle$ is the 3A-type and all $\left\langle a, x^{i}\right\rangle,\left\langle b, x^{i}\right\rangle$ and $\left\langle x^{j}, x^{k}\right\rangle$ are the 2A-type by Lemma A.10, we have obtained a realization of the (2A,3A)-generated subalgebra $X^{[n]}=\left\langle a, b, x^{1}, \ldots, x^{n}\right\rangle$ discussed in Section 4.3 inside $V_{L, \mathbb{R}}^{+}$.

Proposition A.13. $\left\langle a, b, x^{1}, \ldots, x^{n}\right\rangle$ has the Virasoro frame $\eta^{3} \dot{+} \eta^{4} \dot{+} \cdots \dot{+} \eta^{n+4}$. In particular, $\left\langle a, b, x^{1}, \ldots, x^{n}\right\rangle$ is a full subalgebra of $\operatorname{Com}_{V_{L, \mathbb{R}}^{+}}\left\langle\eta^{1}, \eta^{2}, \Omega^{n+5}\right\rangle$.

Proof: Since $\langle a, b\rangle$ is isomorphic to the $3 \mathrm{~A}$-algebra with Virasoro frame $\eta^{3} \dot{+} \eta^{4}$, we have

$$
a+b+c=\frac{15}{32} \eta^{3}+\frac{21}{16} \eta^{4} .
$$

By (A.11) we have

$$
\begin{aligned}
a+b+c & =\frac{15}{32} \eta^{3}+\frac{21}{16} \eta^{4}=\frac{15}{32}\left(s^{3}-s^{2}\right)+\frac{21}{16}\left(s^{4}-s^{3}\right) \\
& =-\frac{15}{32} s^{2}+\frac{3}{16} \sum_{1 \leq i<j \leq 4} w^{-}\left(\epsilon_{i}-\epsilon_{j}\right)+\frac{3}{4} \sum_{1 \leq i \leq 4} w^{-}\left(\epsilon_{i}-\epsilon_{5}\right) .
\end{aligned}
$$

It is clear from expressions that $s^{2}$ and $w^{-}\left(\epsilon_{i}-\epsilon_{j}\right)$ are orthogonal to $w^{-}\left(\epsilon_{5}-\epsilon_{k+5}\right)$ for $1 \leq i<j \leq 4$ and $1 \leq k \leq n$, whereas $\left(w^{-}\left(\epsilon_{i}-\epsilon_{5}\right) \mid w^{-}\left(\epsilon_{5}-\epsilon_{k+5}\right)\right)=2^{-5}$ and one has $w^{-}\left(\epsilon_{i}-\epsilon_{5}\right) \circ w^{-}\left(\epsilon_{5}-\epsilon_{k+5}\right)=w^{-}\left(\epsilon_{i}-\epsilon_{k+5}\right)$ by Lemma A.2. Therefore we have

$$
(a+b+c) \circ x^{k}=-\frac{15}{32} s^{2}+\frac{3}{16} \sum_{1 \leq i<j \leq 4} w^{-}\left(\epsilon_{i}-\epsilon_{j}\right)+\frac{3}{4} \sum_{1 \leq i \leq 4} w^{-}\left(\epsilon_{i}-\epsilon_{k+5}\right) .
$$

So we have

$$
\begin{aligned}
& a+b+c+\sum_{k=1}^{n}(a+b+c) \circ x^{k} \\
& =-\frac{15(n+1)}{32} s^{2}-\frac{3(n+1)}{16} \sum_{1 \leq i<j \leq 4} w^{-}\left(\epsilon_{i}-\epsilon_{j}\right)+\frac{3}{4} \sum_{\substack{1 \leq i \leq 4 \\
5 \leq k \leq n+5}} w^{-}\left(\epsilon_{i}-\epsilon_{k}\right) .
\end{aligned}
$$


Now we consider the conformal vector $\omega^{n}$ of $\left\langle a, b, x^{1}, \ldots, x^{n}\right\rangle$ in (4.14).

$$
\begin{aligned}
& \omega^{n}=\frac{3(3-n)}{2(n+7)} \eta^{3}+\frac{16}{3(n+7)}\left(a+b+c+\sum_{i=1}^{n}(a+b+c) \circ x^{i}\right) \\
& +\frac{4}{n+7}\left(\sum_{i=1}^{n} x^{i}+\sum_{1 \leq j<k \leq n} x^{j} \circ x^{k}\right) \\
& =\frac{3(3-n)}{2(n+7)}\left(\frac{2}{3} \sum_{1 \leq i \leq 4} w^{-}\left(\epsilon_{i}-\epsilon_{j}\right)-s^{2}\right) \\
& +\frac{16}{3(n+7)}\left(-\frac{15(n+1)}{32} s^{2}+\frac{3(n+1)}{16} \sum_{1 \leq i<j \leq 4} w^{-}\left(\epsilon_{i}-\epsilon_{j}\right)+\frac{3}{4} \sum_{\substack{1 \leq i \leq 4 \\
5 \leq k \leq n+5}} w^{-}\left(\epsilon_{i}-\epsilon_{k}\right)\right) \\
& +\frac{4}{n+7}\left(\sum_{i=1}^{n} w^{-}\left(\epsilon_{5}-\epsilon_{i+5}\right)+\sum_{1 \leq j<k \leq n} w^{-}\left(\epsilon_{j+5}-\epsilon_{k+5}\right)\right) \\
& =-s^{2}+\frac{4}{n+7} \sum_{1 \leq i<j \leq n+5} w^{-}\left(\epsilon_{i}-\epsilon_{j}\right)=s^{n+4}-s^{2} .
\end{aligned}
$$

Thus, we have

$$
\omega^{n}=s^{n+4}-s^{2}=\left(s^{3}-s^{2}\right)+\left(s^{4}-s^{3}\right)+\cdots+\left(s^{n+5}-s^{n+4}\right)=\eta^{3} \dot{+} \eta^{4} \dot{+} \cdots \dot{+} \eta^{n+4} .
$$

Since $\omega^{k}$ is the conformal vector of a subalgebra $\left\langle a, b, x^{1}, \ldots, x^{k}\right\rangle$ of $\left\langle a, b, x^{1}, \ldots, x^{n}\right\rangle$ for $1 \leq k \leq n$, the Virasoro vector $\eta^{k+4}=\omega^{k}-\omega^{k-1}$ belongs to $\left\langle a, b, x^{1}, \ldots, x^{n}\right\rangle$. Therefore, $\left\langle a, b, x^{1}, \ldots, x^{n}\right\rangle$ has a Virasoro frame $\omega^{n}=\eta^{3} \dot{+} \eta^{4} \dot{+} \cdots \dot{+} \eta^{n}$.

Denote $Y^{[0]}=\langle a, b\rangle \subset V_{L, \mathbb{R}}^{+}$and $Y^{[k]}=\left\langle a, b, x^{1}, \ldots, x^{k}\right\rangle \subset V_{L, \mathbb{R}}^{+}$for $1 \leq k \leq n$. Then $Y^{[k]}$ provides a realization of $X^{[k]}$ defined in (4.13). Using the Virasoro frame $\eta^{3} \dot{+} \eta^{4} \dot{+} \cdots \dot{+} \eta^{n+4}$ we can prove that the commutant $\operatorname{Com}_{Y[n]} Y^{[n-1]}$ is generated by the $c=c_{n+4}$ Virasoro vector $\eta^{n+4}=\omega^{n}-\omega^{n-1}$ which is denoted by $f^{n}$ in Theorem 4.17. Consider the full subalgebra of $V_{L}$ displayed in (A.13). The irreducible decomposition with respect to this subalgebra is known as the GKO construction. By Eq. (2.20) of [GKO86] (see also Lemma 3.1 of [LLY03]), we have the following decompositions:

$$
\begin{aligned}
& V_{K^{[n+5]}}=\bigoplus_{\substack{1 \leq i_{k} \leq k+1 \\
i_{k} \equiv 1 \bmod 2}} L\left(c_{1}, h_{i_{1}, i_{2}}^{(1)}\right) \otimes \cdots \otimes L\left(c_{n+4}, h_{i_{n+4}, i_{n+5}}^{(n+4)}\right) \otimes L_{\hat{\mathfrak{s}}_{2}}\left(n+5, i_{n+5}-1\right), \\
& V_{K^{[n+5]}+\gamma}=\bigoplus_{\substack{1 \leq j_{k} \leq k+1 \\
j_{k} \equiv 1+\delta_{k} \bmod 2}} L\left(c_{1}, h_{j_{1}, j_{2}}^{(1)}\right) \otimes \cdots \otimes L\left(c_{n+4}, h_{j_{n+4}, j_{n+5}}^{(n+4)}\right) \otimes L_{\hat{\mathfrak{s}}_{2}}\left(n+5, j_{n+5}-1\right),
\end{aligned}
$$

where $\delta_{1}=1, \delta_{2}=0, \delta_{3}=1$ and $\delta_{k}=0$ for $4 \leq k \leq n+5$. By the decompositions above, we see that the commutant of $\left\langle\eta^{1}, \eta^{2}, \ldots, \eta^{n+3}, \Omega^{n+5}\right\rangle$ in $V_{L, \mathbb{R}}$ is exactly $\left\langle\eta^{n+4}\right\rangle$. Therefore, $\operatorname{Com}_{Y^{[n]}} Y^{[n-1]}$ is generated by the $c=c_{n+4}$ Virasoro vector $\eta^{n+4}=\omega^{n}-\omega^{n-1}$. 
Conjecture A.14. The subalgebra $X^{[n]}=\left\langle a, b, x^{1}, \ldots, x^{n}\right\rangle$ defined in (4.13) is unique up to isomorphism and coincides with the subalgebra $\operatorname{Com}_{V_{L, \mathbb{R}}^{+}}\left\langle\eta^{1}, \eta^{2}, \Omega^{n+5}\right\rangle$ of $V_{L, \mathbb{R}}^{+}$. In particular, $\operatorname{Com}_{X^{[n]}} X^{[n-1]}$ is generated by the $c=c_{n+4}$ Virasoro vector $f^{n}$ in Theorem 4.17 .

Remark A.15. We will construct $V^{\natural}$ as an extension of $\operatorname{Com}_{V_{L, \mathbb{R}}^{+}}\left\langle\eta^{1}, \eta^{2}, \Omega^{n+5}\right\rangle$ in [CLY].

Finally, we prove that the Virasoro vectors in (4.16) are linearly independent. By (A.16) and (A.17), we know that the following vectors are linearly independent in $V_{K^{[n+5]}}$.

$$
\begin{aligned}
& u_{a, b}=\eta^{3}, \quad x^{i}=w^{-}\left(\epsilon_{5}-\epsilon_{i+5}\right), \quad a=w^{-}\left(\epsilon_{4}-\epsilon_{5}\right), \quad a \circ x^{i}=w^{-}\left(\epsilon_{4}-\epsilon_{i+5}\right), \\
& a+b+c, \quad(a+b+c) \circ x^{i}, \quad x^{j} \circ x^{k}=w^{-}\left(\epsilon_{j+5}-\epsilon_{k+5}\right), \quad 1 \leq i \leq n, \quad 1 \leq j<k \leq n .
\end{aligned}
$$

On the other hand, by (A.14) and (A.15) we have

$$
\begin{aligned}
q=\frac{2^{6}}{\sqrt{3}}(b-c)= & \sum_{k=1}^{3}\left(2 \gamma+\epsilon_{k}-\epsilon_{4}-4 \epsilon_{5}\right)_{(-1)}\left(e^{\gamma-\epsilon_{k}-\epsilon_{4}}-e^{-\gamma+\epsilon_{k}+\epsilon_{4}}\right) \\
& \quad-4 \sum_{k=1}^{3}\left(e^{\gamma-\epsilon_{k}-\epsilon_{5}}+e^{-\gamma+\epsilon_{k}+\epsilon_{5}}\right)+12\left(e^{\gamma-\epsilon_{4}-\epsilon_{5}}+e^{-\gamma+\epsilon_{4}+\epsilon_{5}}\right) .
\end{aligned}
$$

Since $\sigma_{x^{i}}$ acts on $V_{L}$ by a reflection associated to $\epsilon_{5}-\epsilon_{i+5}$, we have

$$
\begin{aligned}
\frac{2^{6}}{\sqrt{3}}(b-c) \circ x^{i}=\sum_{k=1}^{3} & \left(2 \gamma+\epsilon_{k}-\epsilon_{4}-4 \epsilon_{i+5}\right)_{(-1)}\left(e^{\gamma-\epsilon_{k}-\epsilon_{4}}-e^{-\gamma+\epsilon_{k}+\epsilon_{4}}\right) \\
& \quad-4 \sum_{k=1}^{3}\left(e^{\gamma-\epsilon_{k}-\epsilon_{i+5}}+e^{-\gamma+\epsilon_{k}+\epsilon_{i+5}}\right)+12\left(e^{\gamma-\epsilon_{4}-\epsilon_{i+5}}+e^{-\gamma+\epsilon_{4}+\epsilon_{i+5}}\right) .
\end{aligned}
$$

From the expressions above, we see that $b-c$ and $(b-c) \circ x^{i}, 1 \leq i \leq n$, are linearly independent in $V_{K^{[n+5]}+\gamma}$. Thus, by considering the Gram matrix, all the Virasoro vectors in (4.16) are also linearly independent.

\section{A.4 Construction of $\langle a, b, x, y\rangle$ with $\langle x, y\rangle \cong U_{3 \mathrm{~A}}$}

In this subsection, we will construct explicitly four Ising vectors $a, b, x, y$ in the VOA $V_{\Lambda}^{+}$ associated to the Leech lattice $\Lambda$ such that $\langle a, b\rangle \cong\langle x, y\rangle \cong U_{3 \mathrm{~A}}$ and $\langle a, x\rangle \cong\langle b, x\rangle \cong$ $\langle a, y\rangle \cong\langle b, y\rangle \cong U_{2 \mathrm{~A}}$. Therefore, the Griess algebra discussed in Section 4.2 does exist.

For explicit calculations, we will use the notion of hexacode balance (or MOG) to denote the codewords of the Golay code $\mathcal{G}$ and the vectors in the Leech lattice $\Lambda$ [CS99, G98b]. Namely, we arrange the set $\Omega=\{1,2, \ldots, 24\}$ into a $4 \times 6$ array such that the six columns forms a sextet. Recall that the Conway group $\mathrm{Co}_{0}$ is the orthogonal group $\mathrm{O}(\Lambda)$ of $\Lambda$ and contains 4 conjugacy classes of involutions (see [CS99]). If $i$ is an involution with trace 8 on $\Lambda$, then the $(-1)$-eigenlattice is isomorphic to $\sqrt{2} E_{8}$.

Consider the following 4 involutions in $\mathrm{O}(\Lambda)$. 

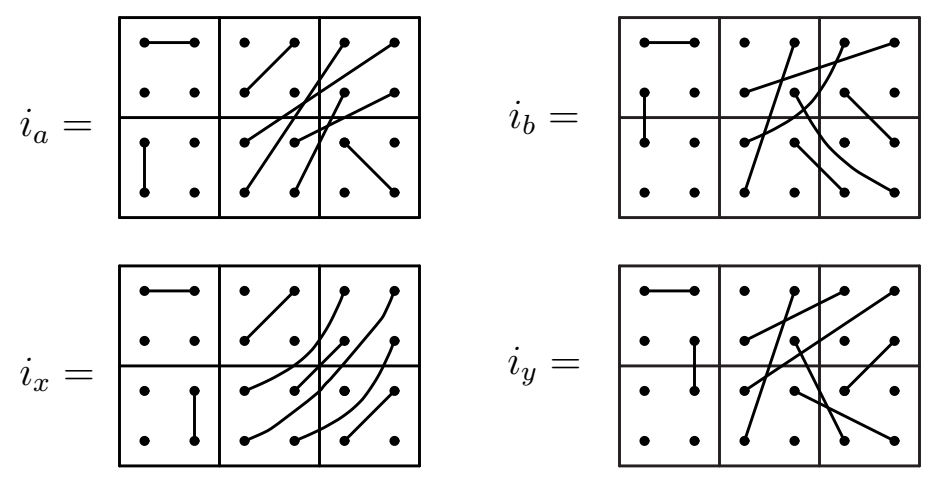

The automorphisms above should be viewed as permutations on 24 coordinates and they act on the Leech lattice $\Lambda$ from the left.

Note that
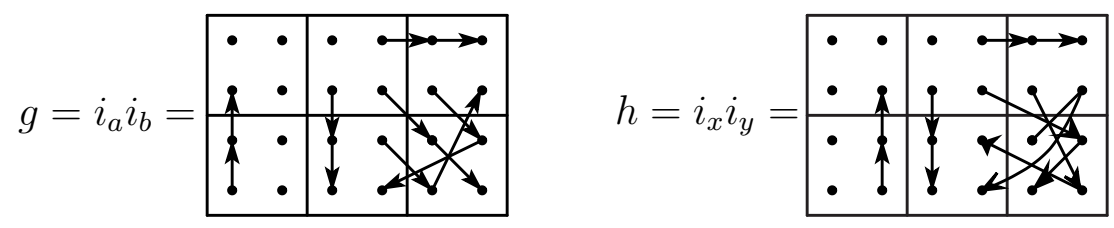

where a composition of above permutations is executed from right to left.

We have $\left\langle i_{a}, i_{b}\right\rangle \cong\left\langle i_{x}, i_{y}\right\rangle \cong \mathrm{S}_{3}$. Note that $i_{x}$ and $i_{y}$ commute with $\left\langle i_{a}, i_{b}\right\rangle$. Thus, we also have $\left\langle i_{a}, i_{b}, i_{x}\right\rangle \cong\left\langle i_{a}, i_{b}, i_{y}\right\rangle \cong \operatorname{Dih}_{12}$, a dihedral group of order 12 .

Notation A.16. Let $A, B, X, Y$ be the (-1)-eigenlattices of $i_{a}, i_{b}, i_{x}$ and $i_{y}$, respectively. Then $A \cong B \cong X \cong Y \cong \sqrt{2} E_{8}$. Moreover, $A+B \cong X+Y \cong \operatorname{DIH}_{6}(14)$ and $A+B+X \cong$ $A+B+Y \cong \mathrm{DIH}_{12}(16)$ by the analysis in [GL11].

Notation A.17 (cf. Remark A.4). For any $\sqrt{2} E_{8}$-sublattice $M$, let

$$
t_{M}=\frac{1}{16} \omega_{M}+\frac{1}{32} \sum_{\substack{\alpha \in M \\(\alpha \mid \alpha)=4}} e^{\alpha}
$$

be the standard Ising vector in $V_{M}^{+}$.

Since $A$ and $B$ are doubly even, it is possible to choose a 2-cocycle $\varepsilon \in \mathbb{Z}^{2}(\Lambda,\{ \pm 1\})$ such that $\varepsilon$ is trivial on $A$ and $B$ (see [LY14, Notation 5.38]). Let $a:=t_{A}$ and $b:=t_{B}$ be the standard Ising vector in $V_{A}^{+}$and $V_{B}^{+}$, respectively.

Lemma A.18. Let $M, N, E$ be $\sqrt{2} E_{8}$-sublattices of the Leech lattice. Suppose $M+N \cong$ $\mathrm{DIH}_{6}(14)$ and $M+E \cong N+E \cong \mathrm{DIH}_{4}(12)$. Then there exists an Ising vector $e \in V_{E}^{+}$ such that $\left(t_{M} \mid e\right)=\left(t_{N} \mid e\right)=2^{-5}$. 
Proof: Let $i_{L}$ be the SSD involution associated to a $\sqrt{2} E_{8}$-sublattice $L$. Since $i_{E} i_{N}$ has order 2 and trace 8 , the $(-1)$-eigenlattice of $i_{E} i_{N}$ is isometric to $\sqrt{2} E_{8}$. Let $N^{\prime}$ be the $(-1)$ eigenlattice of $i_{E} i_{N}$. Then $\left\langle i_{M}, i_{N} i_{E}\right\rangle \cong \operatorname{Dih}_{12}$ and hence $M+N^{\prime} \cong M+N+E \cong \operatorname{DIH}_{12}(16)$ as defined in [GL11]. Let $e^{\prime}$ be an Ising vector of $E_{8}$-type in $V_{N^{\prime}}^{+}$(see Remark A.4 for definition). Then $\left\langle t_{M}, e^{\prime}\right\rangle \cong U_{6 \mathrm{~A}}$ since $\tau_{t_{M}} \tau_{e^{\prime}}$ has order 6 .

Let $\tilde{e}$ be the central Ising vector in $\left\langle t_{M}, e^{\prime}\right\rangle \cong U_{6 \mathrm{~A}}$ (cf. Eq. (2.9)). Then $\tilde{e} \in V_{E}^{+}$. Let $\tilde{t}_{N}=e^{\prime} \circ t_{N}$. Then $\tilde{t}_{N} \in V_{N}^{+}$and

$$
\left(t_{M} \mid \tilde{e}\right)=\left(\tilde{t}_{N} \mid \tilde{e}\right)=\frac{1}{32} \quad \text { and } \quad\left(t_{M} \mid \tilde{t}_{N}\right)=\frac{13}{2^{10}} .
$$

By the classification of Ising vectors in [LSY07] and [LS07] (cf. Remark A.4), $\tilde{t}_{N}=\varphi_{x}\left(t_{N}\right)$ for some $x \in N^{*}$. Moreover, by Proposition 3.4 of [GL12b], there exists an $\alpha \in M$ such that $\left.\varphi_{\alpha}\right|_{V_{N}^{+}}=\varphi_{x}$. Hence, we have

$$
\varphi_{\alpha}\left(t_{M}\right)=t_{M} \quad \text { and } \quad \varphi_{\alpha}\left(\tilde{t}_{N}\right)=\varphi_{\alpha} \varphi_{x}\left(t_{N}\right)=t_{N}
$$

Now let $e=\varphi_{\alpha}(\tilde{e})$. Then we have

$$
\left(t_{M} \mid e\right)=\left(t_{N} \mid e\right)=\frac{1}{32}
$$

by Equation (A.20).

Finally, by Lemma A.18, there exist Ising vectors $x \in V_{X}^{+}$and $y \in V_{Y}^{+}$such that $\langle a, x\rangle \cong\langle b, x\rangle \cong\langle a, y\rangle \cong\langle b, y\rangle \cong U_{2 \mathrm{~A}}$. Since $X+Y \cong \operatorname{DIH}_{6}(14)$, we have $(x \mid y)=13 \cdot 2^{-10}$ or $5 \cdot 2^{-10}$. By Theorem 3.5, the case $(x \mid y)=5 \cdot 2^{-10}$ is impossible. Hence we have $(x \mid y)=13 \cdot 2^{-10}$ and $\langle x, y\rangle \cong U_{3 \mathrm{~A}}$ as desired.

\section{References}

[ATLAS] J.H. Conway, R.T. Curtis, S.P. Norton, R.A. Parker and R.A. Wilson, ATLAS of finite groups. Clarendon Press, Oxford, 1985.

[As97] M. Aschbacher, 3-transposition groups. Cambridge Tracts in Mathematics, 124. Cambridge University Press, Cambridge, 1997.

[B86] R.E. Borcherds, Vertex algebras, Kac-Moody algebras and the Monster. Proc. Nat. Acad. Sci. USA 83 (1986), 3068-3071.

[C85] J.H. Conway, A simple construction for the Fischer-Griess Monster group. Invent. Math. 79 (1985), 513-540.

[CS99] J.H. Conway and N. J. A. Sloane, Sphere packings, lattices and groups. 3rd Edition, Springer, New York, 1999.

[CH95] H. Cuypers and J.I. Hall, The 3-transposition groups with trivial center. J. Algebra 178, (1995), 149-193. 
[CLY] T. Creutzig, C.H. Lam and H. Yamauchi, An exceptional construction of the moonshine vertex operator algebra, in preparation.

[DJY19] C. Dong, X. Jiao and N. Yu, 6A-Algebra and its representations. arXiv:1902.06951.

[DLMN98] C. Dong, H. Li, G. Mason and S.P. Norton, Associative subalgebras of Griess algebra and related topics. Proc. of the Conference on the Monster and Lie algebra at the Ohio State University, May 1996, ed. by J. Ferrar and K. Harada, Walter de Gruyter, Berlin - New York, 1998.

[DLY09] C. Dong. C.H. Lam and H. Yamada, W-algebras related to parafermion algebras. J. Algebra 322 (2009), no.7, 2366-2403.

[DMZ94] C. Dong, G. Mason and Y. Zhu, Discrete series of the Virasoro algebra and the moonshine module. Proc. Symp. Pure. Math., American Math. Soc. 56 II (1994), 295-316.

[DW16] C. Dong and Q. Wang, Quantum dimensions and fusion rules for parafermion vertex operator algebras. Proc. Amer. Math. Soc. 144 (2016), no. 4, 1483-1492.

[Fi71] B. Fischer, Finite groups generated by 3-transpositions. I. Invent. Math. 13, 232-246.

[FLM88] I.B. Frenkel, J. Lepowsky and A. Meurman, Vertex Operator Algebras and the Monster. Academic Press, New York, 1988.

[FZ92] I.B. Frenkel and Y. Zhu, Vertex operator algebras associated to representation of affine and Virasoro algebras. Duke Math. J. 66 (1992), 123-168.

[GKO86] P. Goddard, A. Kent and D. Olive, Unitary representations of the Virasoro and super-Virasoro algebras. Comm. Math. Phys. 103 (1986), 105-119.

[G82] R.L. Griess, The friendly giant. Invent. Math. 69 (1982), 1-102.

[G98a] R.L. Griess, A vertex operator algebra related to $E_{8}$ with automorphism group $\mathrm{O}^{+}(10,2)$. The Monster and Lie algebras (Columbus, OH, 1996), 43-58, Ohio State Univ. Math. Res. Inst. Publ., 7, de Gruyter, Berlin, 1998.

[G98b] R.L. Griess, Twelve Sporadic Groups. Springer Verlag, 1998.

[GL11] R.L. Griess and C. H. Lam, $E E_{8}$ lattices and dihedral groups. Pure and Applied Math Quarterly (special issue for Jacques Tits), 7 (2011), no. 3, 621-743.

[GL12a] R.L. Griess and C.H. Lam, Diagonal lattices and rootless $E E_{8}$ pairs. J. Pure and Appl. Algebra 216 (2012), no. 1, 154-169.

[GL12b] R.L. Griess and C.H. Lam, Moonshine paths for 3A and 6A nodes of the extended E-diagram. J. Algebra 379 (2013), 85-112. doi:10.1016/j.jalgebra.2012.12.019

[H10] G. Höhn, The group of symmetries of the shorter moonshine module. Abh. Math. Semin. Univ. Hambg. 80 (2010), no. 2, 275-283.

[HLY12a] G. Höhn, C.H. Lam and H. Yamauchi, McKay's $E_{7}$ observation on the Baby Monster. Internat. Math. Res. Notices (2012), doi:10.1093/imrn/rnr009. 
[HLY12b] G. Höhn, C.H. Lam and H. Yamauchi, McKay's $E_{6}$ observation on the largest Fischer group. Comm. Math. Physics 310 Vol. 2 (2012), 329-365.

[HRS15] J.I. Hall, F. Rehren and S. Shpectorov, Primitive axial algebras of Jordan type. J. Algebra 437 (2015), 79-115.

[I76] I. Martine Isaacs, Character theory of finite groups. Pure and Applied Mathematics, No. 69. Academic Press, New York-London, 1976.

[KMY00] M. Kitazume, M. Miyamoto and H. Yamada, Ternary codes and vertex operator algebras. J. Algebra 223 (2000), 379-395.

[LLY03] C.H. Lam, N. Lam and H. Yamauchi, Extension of unitary Virasoro vertex operator algebra by a simple module. Internat. Math. Res. Notices 11 (2003), 577-611.

[LM06] C.H. Lam and M. Miyamoto, Niemeier lattices, Coxeter elements, and McKay's E - $^{-}$ observation on the Monster simple group. Internat. Math. Res. Notices Article ID 35967 (2006), 1-27.

[LSY07] C.H. Lam, S. Sakuma and H. Yamauchi, Ising vectors and automorphism groups of commutant subalgebras related to root systems. Math. Z. 255 vol.3 (2007), 597-626.

[LS07] C.H. Lam and H. Shimakura, Ising vectors in the vertex operator algebra $V_{\Lambda}^{+}$associated with the Leech lattice $\Lambda$. Internat. Math. Res. Notices 2007 Art. ID rnm132, $21 \mathrm{pp}$.

[LY04] C.H. Lam and H. Yamada, Decomposition of the lattice vertex operator algebra $V_{\sqrt{2} A_{l}}$.J. Algebra $272(2004), 614-624$.

[LYY07] C.H. Lam, H. Yamada and H. Yamauchi, Vertex operator algebras, extended $E_{8-}$ diagram, and McKay's observation on the Monster simple group. Trans. Amer. Math. Soc. 359 (2007), 4107-4123.

[LYY05] C.H. Lam, H. Yamada and H. Yamauchi, McKay's observation and vertex operator algebras generated by two conformal vectors of central charge 1/2. Internat. Math. Res. Papers 3 (2005), 117-181.

[LY07] C.H. Lam and H. Yamauchi, A characterization of the moonshine vertex operator algebra by means of Virasoro frames. International Mathematics Research Notices 2007 (2007), article ID:rnm003, 9 pages.

[LY14] C.H. Lam and H. Yamauchi, On 3-transposition groups generated by $\sigma$-involutions associated to $c=4 / 5$ Virasoro vectors. J. Algebra, 416 (2014), 84-121.

[LY16] C.H. Lam and H. Yamauchi, 3-dimensional Griess algebras and Miyamoto involutions. Preprint. arXiv: 1604.04470.

[LSu] C. H. Lam and C.S. Su, Griess algebra generated by two $3 A$-algebras with a common axis. J. Math. Soc. Japan 67 (2015), 453-476.

[Ma01] A. Matsuo, Norton's trace formulae for the Griess algebra of a vertex operator algebra with larger symmetry. Commun. Math. Phys. 224 (2001), 565-591. 
[Ma05] A. Matsuo, 3-transposition groups of symplectic type and vertex operator algebras. J. Math. Soc. Japan 57 (2005), no. 3, 639-649. arXiv:math/0311400.

[MN93] W. Meyer and W. Neutsch, Associative subalgebras of the Griess algebra. J. Algebra 158 (1993), 1-17.

[Mi96] M. Miyamoto, Griess algebras and conformal vectors in vertex operator algebras. $J$. Algebra 179 (1996), 528-548.

[Mi01] M. Miyamoto, 3-state Potts model and automorphisms of vertex operator algebras of order 3. J. Algebra 239 (2001), 56-76.

[Mi03] M. Miyamoto, VOAs generated by two conformal vectors whose $\tau$-involutions generate $\mathrm{S}_{3}$. J. Algebra 268 (2003), 653-671.

[Mi04] M. Miyamoto, A new construction of the moonshine vertex operator algebra over the real number field. Ann. Math. 159 (2004), 535-596.

[R15] F. Rehren, Linear idempotents in Matsuo algebras. To appear in Indiana. Uni. Math. $J$.

[S07] S. Sakuma, 6-transposition property of $\tau$-involutions of vertex operator algebras. Internat. Math. Res. Notices 2007, no. 9, Art. ID rnm 030, 19 pp.

[SY03] S. Sakuma and H. Yamauchi, Vertex operator algebra with two Miyamoto involutions generating $S_{3}$. J. Algebra 267 (2003), 272-297.

[W93] W. Wang, Rationality of Virasoro vertex operator algebras. Internat. Math. Res. Notices 71 (1993), 197-211.

[Y14] H. Yamauchi, Extended Griess algebras and Matsuo-Norton trace formulae. Conformal Field Theory, Automorphic Forms and Related Topics, Springer, 2014, 75-107. 